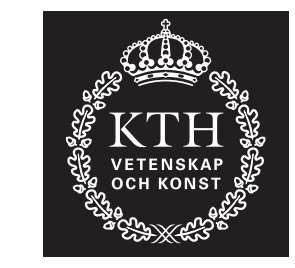

KTH Computer Science

\title{
Doubly-fed Induction Generator Modeling and Control in DigSilent Power Factory
}

\author{
CAMILLE HAMON
}

Master's Thesis at KTH School of Electrical Engineering

XR-EE-ES 2010:004 



\begin{abstract}
International agreements have set high demands on the share of renewable energy in the total energy mix. From the different renewable sources, significant investments are made in wind power. More and more wind turbines are being built and their number is due to rise dramatically. There are many different generator technologies, but this paper focuses on the doubly-fed induction generator (DFIG).

DFIGs are generators which are connected to the grid on both stator and rotor sides. The machine is controlled via converters connected between the rotor and the grid. The size of these converters determines the speed range of the DFIG.

Wind farm connections to the grid must satisfy grid requirements set by transmission system operators. This means that the study of their dynamic responses to disturbances has become a critical issue, and is becoming increasingly important for induction generators, due to their growing size and number.

Several computer programs exist to carry out dynamical simulations and this work will focus on one of them, namely Power Factory from DigSilent. It offers a large choice of builtin components. These components can be controlled through input signals. It is therefore possible for the user to design control strategies.

Power Factory has two models of DFIG. A new model has also been developed, based upon a controllable voltage source. These three models are compared, in terms of dynamical behavior and simulation time. One is then used to study the effect of introducing a certain signal to the control strategy.
\end{abstract}





\section{Acknowledgments}

Many people have contributed to this work.

First, I would like to thank Katherine and Mehrdad for their supervision and advice which have guided me all along the thesis. The whole department of Electric Power System should be thanked as well for all the fika and other distractions from work. Special gratitude goes of course to the Bubenkorummet for all the nice moments.

I am grateful to my family and friends who have supported me in the best possible way.

Finally, special warm thoughts go to a person who has managed the impossible task to be there when needed, while being thousands of kilometers away. 


\section{Contents}

$\begin{array}{ll}\text { List of Symbols } & \text { ix }\end{array}$

List of Figures $\quad$ xi

List of Tables $\quad$ xii

1 Introduction 1

1.1 Background . . . . . . . . . . . . . . . . . . . . . . . 1

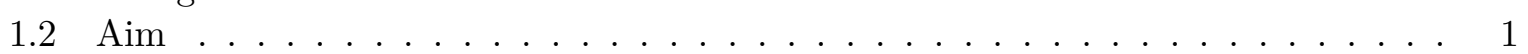

1.3 Different types of lavout $\ldots \ldots \ldots \ldots \ldots \ldots \ldots \ldots$

1.4 Different tvpes of generator $\ldots \ldots \ldots \ldots \ldots \ldots \ldots$

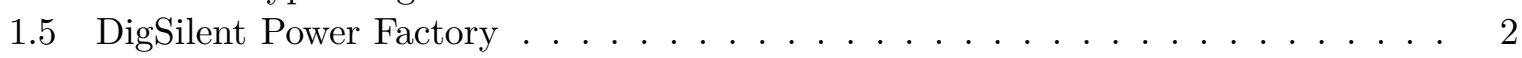

1.6 Layout of this work . . . . . . . . . . . . . . . . . . 2

2 Doubly fed induction generators 3

2.1 Working Principles . . . . . . . . . . . . . . . . . . . . . . 3

2.2 Modeling of doubly fed induction generators . . . . . . . . . . . . . 4

2.2.1 Electrical relations . . . . . . . . . . . . . . . . . . . . 4

2.2 .2 Mechanical relations . . . . . . . . . . . . . . . . 5

$2.3 d a 0$-reference frame $\ldots \ldots \ldots \ldots \ldots \ldots \ldots \ldots$

2.4 Modeling in $d q 0$-reference frame $\ldots \ldots \ldots \ldots \ldots \ldots$

$2.4 .1 \quad$ Electrical equations $\ldots \ldots \ldots \ldots \ldots$. . . . . . . . . . . . 7

2.4 .2 Phasor notation . . . . . . . . . . . . . . . . . . 9

2.4 .3 Mechanical equations . . . . . . . . . . . . . . . . . . . . 10

2.5 Per-unit System . . . . . . . . . . . . . . . . . . . . . . . 11

$\begin{array}{lll}3 & \text { Reduced order models } & 15\end{array}$

3.1 Third order model . . . . . . . . . . . . . . . . . . . . . . . . 15

3.1 .1 Neglecting the stator transients . . . . . . . . . . . . . . . . . 15

3.1 .2 State space formulation . . . . . . . . . . . . . . . 16

3.1 .3 Neglecting the stator resistance . . . . . . . . . . . . . . . . . . . . . 17

3.1 .4 Power considerations . . . . . . . . . . . . . . . . . . . . . . 18

3.1 .5 Mechanical equation . . . . . . . . . . . . . . . . . . . . . . . . . . . . . . . . . . .

3.2 First order model . . . . . . . . . . . . . . . . . . . . . 18

\begin{tabular}{|lll}
4 & Converter models & 19
\end{tabular}

4.1 Supply-side converter . . . . . . . . . . . . . . . . . . . . . . . . . . 19

4.2 Rotor-side converter . . . . . . . . . . . . . . . . . . 20

$\begin{array}{lll}5 \text { Control strategy } & 21\end{array}$ 
5.1 Controlling active power . . . . . . . . . . . . . . . . . . . . . . 21

5.2 Controlling reactive power or voltage . . . . . . . . . . . . . . . . 21

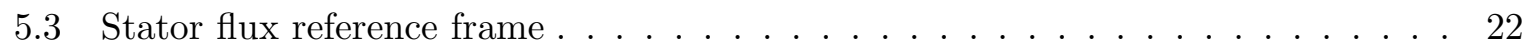

5.4 Acting upon rotor currents . . . . . . . . . . . . . . . . . . . . 23

5.5 Control blocks . . . . . . . . . . . . . . . . . . . . . 24

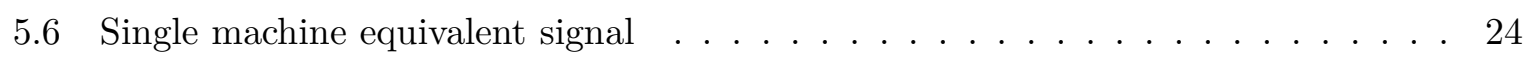

5.7 Switching between reference frames . . . . . . . . . . . . . . . . . . 25

\begin{tabular}{lll}
\hline & DFIG model & 27
\end{tabular}

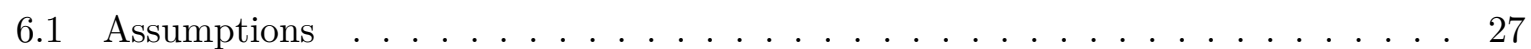

6.2 Equivalent circuit . . . . . . . . . . . . . . . . . . . . . . 27

6.3 First- and third-order models . . . . . . . . . . . . . . . . . . 28

$6.3 .1 \quad$ First-order model . . . . . . . . . . . . . . . . . . . . . . . 29

6.3 .2 Third-order model . . . . . . . . . . . . . . . . . . . . . 30

6.4 Theoretical power delivered by the first order model . . . . . . . . . . . . . . 30

6.4 .1 Calculation of the stator active power . . . . . . . . . . . 30

6.4 .2 Calculation of the stator reactive power . . . . . . . . . . . . . 30

6.4.3 Calculation of the rotor active power . . . . . . . . . . . . . . 31

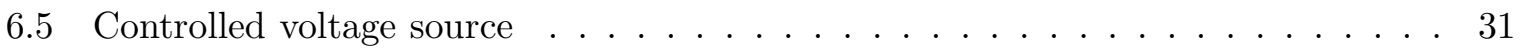

6.6 Load flow calculations $\ldots \ldots \ldots \ldots$

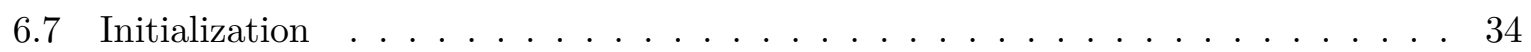

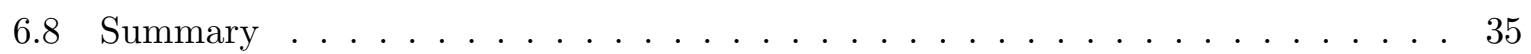

\begin{tabular}{lll}
\hline 7 & Built-in models & 37
\end{tabular}

$\begin{array}{lll}8 & \text { Simulations } & 41\end{array}$

8.1 Simulation setup . . . . . . . . . . . . . . . . . . . . . . . . . 41

8.1 .1 Grid . . . . . . . . . . . . . . . . . . . . . . . . 41

8.1 .2 Values used in the simulations . . . . . . . . . . . . . . . 41

8.1 .3 Studv cases . . . . . . . . . . . . . . . . . . . . . . . . 41

8.2 Model comparison . . . . . . . . . . . . . . . . . . . . . . . . 43

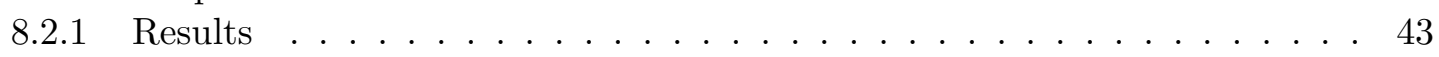

8.2 .2 Simulation speed $\ldots \ldots \ldots \ldots \ldots \ldots$

8.2 .3 Comments . . . . . . . . . . . . . . . . . . . 50

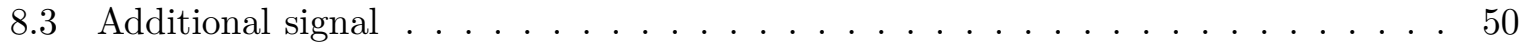

$\begin{array}{lll}9 \text { Conclusion } & 59\end{array}$

\begin{tabular}{ll}
\hline Appendices & 61
\end{tabular}

\begin{tabular}{|ll}
\hline A Values used in the simulation & 63
\end{tabular}

\begin{tabular}{|ll}
\hline B PowerFactory details & 65
\end{tabular}

\begin{tabular}{lr}
\hline Bibliography & 67
\end{tabular} 



\section{List of Symbols}

The reader can refer to the list below to find any important notation used in this work.

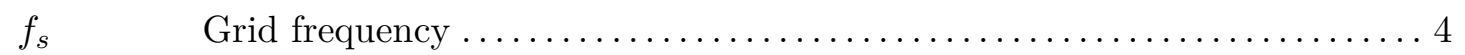

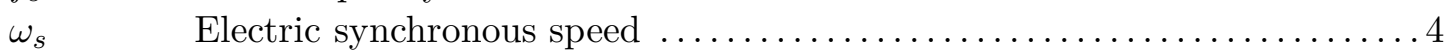

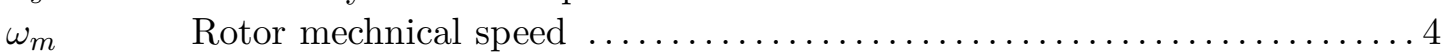

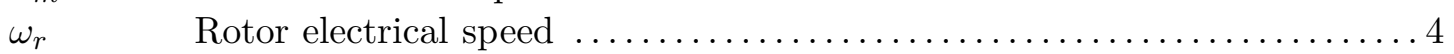

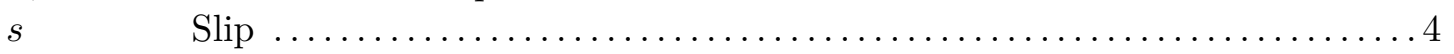

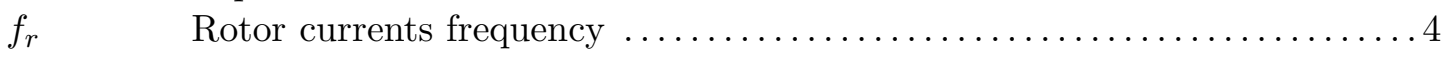

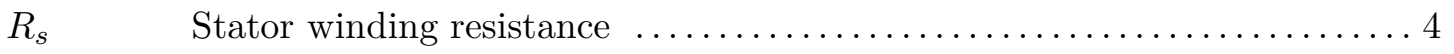

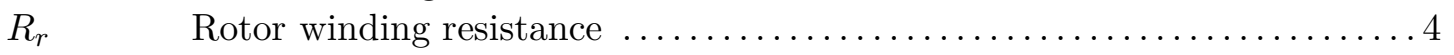

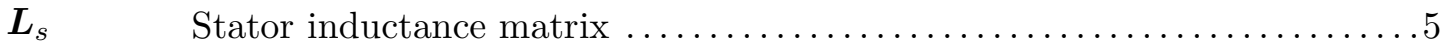

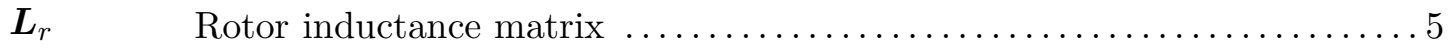

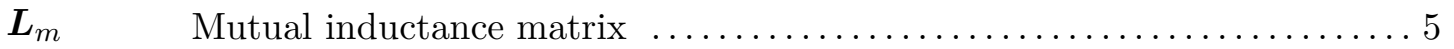

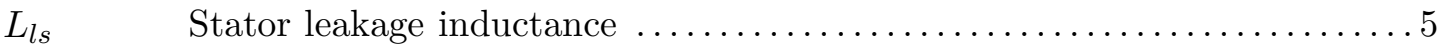

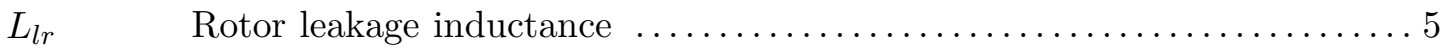

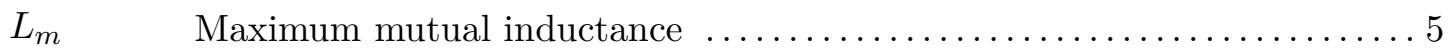

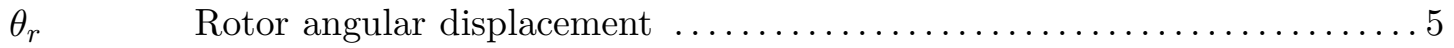

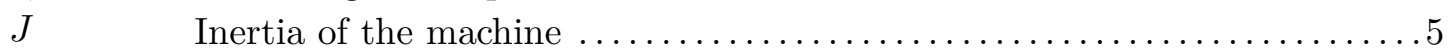

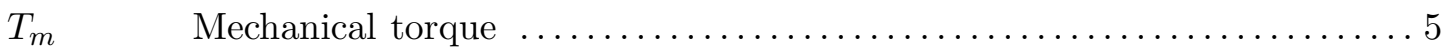

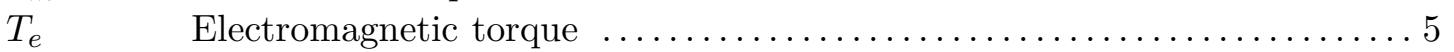

$\beta_{d q} \quad$ Angular displacement of the dq0-reference frame $\ldots \ldots \ldots \ldots \ldots \ldots \ldots$. 5

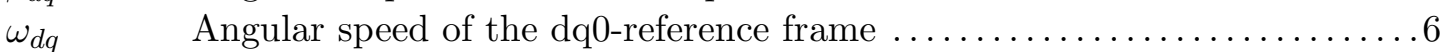

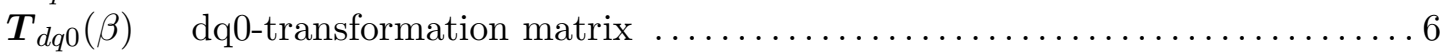

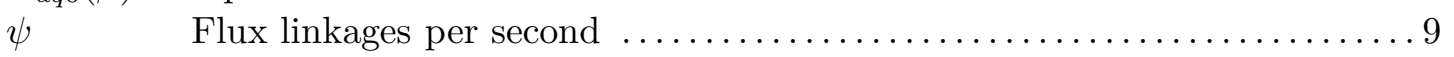

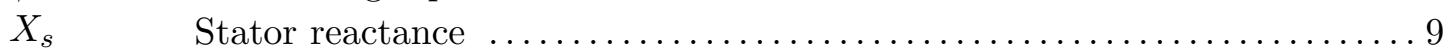

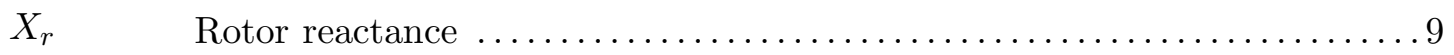

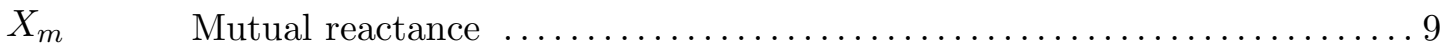

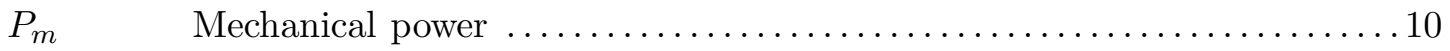

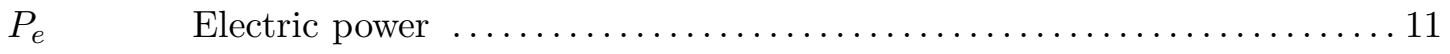

$P_{t} \quad$ Power available on the generator shaft $\ldots \ldots \ldots \ldots \ldots \ldots \ldots \ldots \ldots \ldots \ldots \ldots$ 10

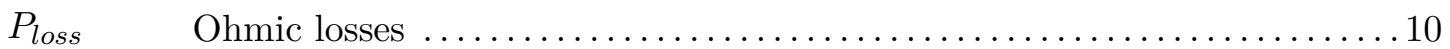

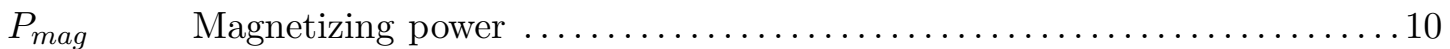

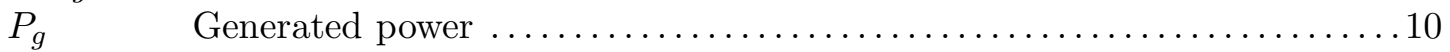

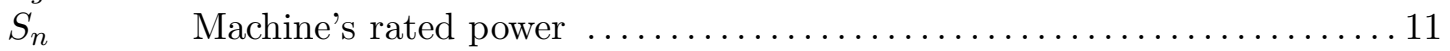

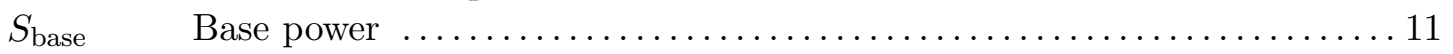

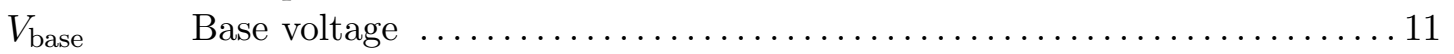

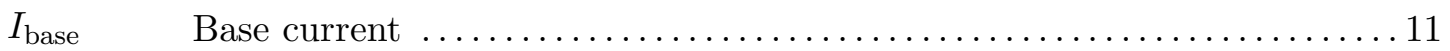

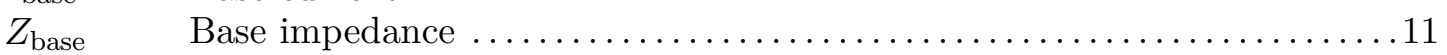

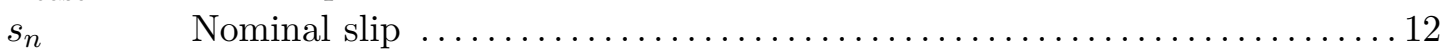

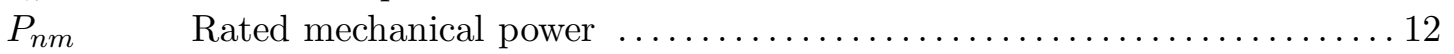




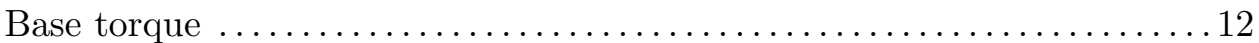

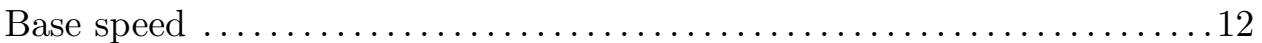

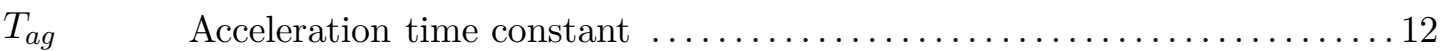

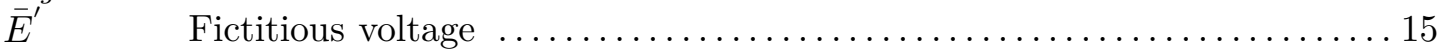

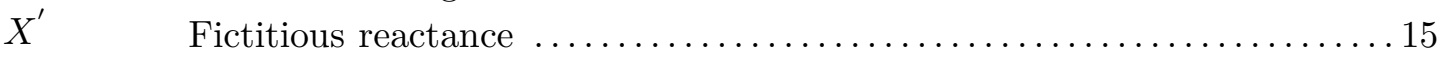

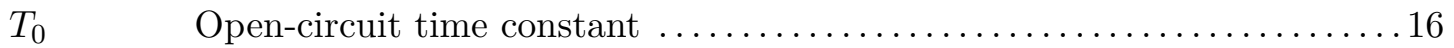

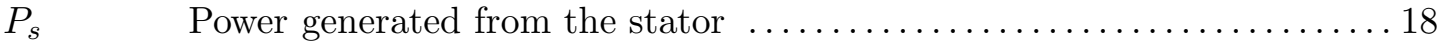

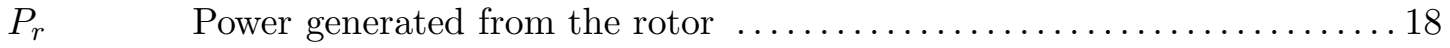

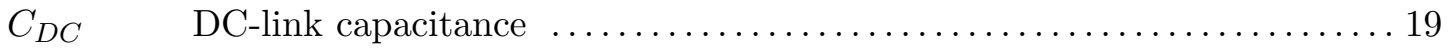

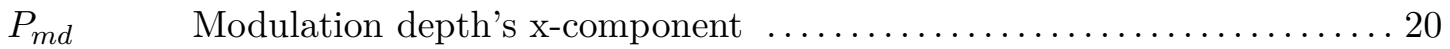

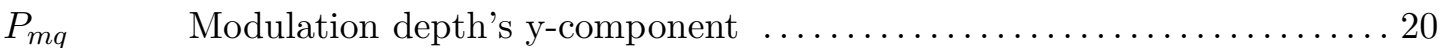

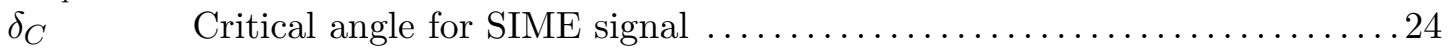

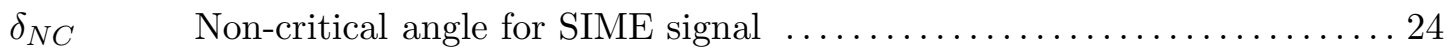

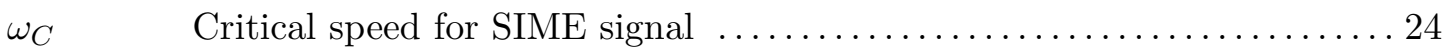

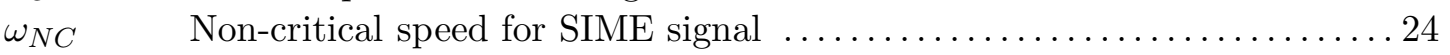

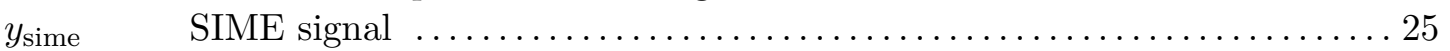

$K_{S p} \quad$ Amplification gain for the SIME signal on the $\mathrm{P}$ side $\ldots \ldots \ldots \ldots \ldots \ldots .25$

$K_{S q} \quad$ Amplification gain for the SIME signal on the Q side $\ldots \ldots \ldots \ldots \ldots \ldots \ldots .25$

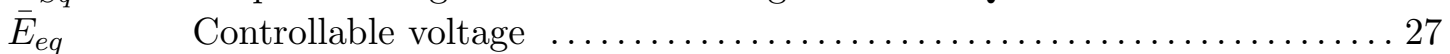

$X_{e q} \quad$ Reactance associated to the controllable voltage $\ldots \ldots \ldots \ldots \ldots \ldots \ldots \ldots \ldots \ldots \ldots$

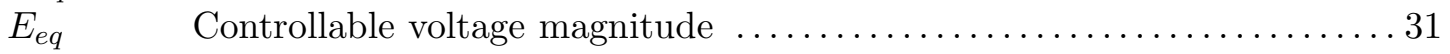

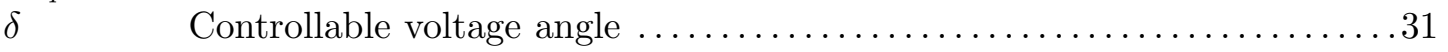




\section{List of Figures}

2.1 DFIG with its converters $\ldots \ldots \ldots \ldots \ldots \ldots \ldots$

2.2 Relation between the different reference frames $\ldots \ldots \ldots \ldots$. . . . . . . . . . . . . . . . . . . . .

2.3 DFIG equivalent circuit $\ldots \ldots \ldots \ldots \ldots \ldots$

3.1 Stator equivalent circuit with stator flux transients neglected . . . . . . . . . . . 16

3.2 Stator equivalent circuit with rotor resistance neglected $\ldots \ldots \ldots$. . . . . . . 17

4.1 Grid-side model . . . . . . . . . . . . . . . . . . . . . . . . . . . . 19

4.2 Rotor-side model . . . . . . . . . . . . . . . . . . . . . . . . . 20

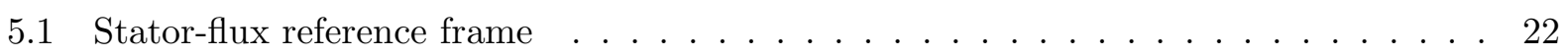

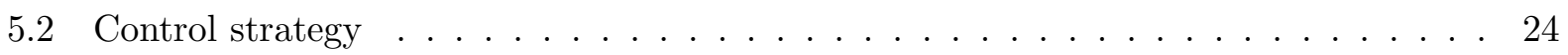

5.3 Control strategy with additional signal . . . . . . . . . . . . . . . . . 25

5.4 Different reference frames $\ldots \ldots \ldots \ldots \ldots \ldots \ldots$

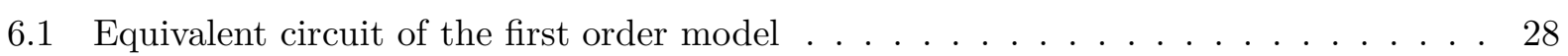

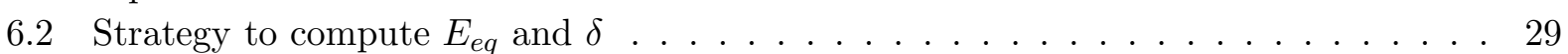

6.3 User-created model in Power Factory . . . . . . . . . . . . . . . . . . . . . . . . 33

6.4 Theoretical power calculation process $\ldots \ldots \ldots \ldots \ldots \ldots$

7.1 Built-in model with DC link in Power Factory . . . . . . . . . . . . . . . . . . 37

7.2 Built-in model without DC link in Power Factory . . . . . . . . . . . . . . . . 38

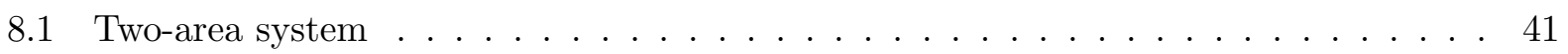

8.2 Turbine governor in Power Factory $\ldots \ldots \ldots$. . . . . . . . . . . . . . . . 42

8.3 Excitation system with PSS $\ldots \ldots \ldots \ldots \ldots$. . . . . . . . . . . . . . . . . . 43

8.4 Model comparison-disturbance $1 \ldots \ldots \ldots \ldots \ldots$. . . . . . . . . . . . 44

8.5 Model comparison - disturbance 2 . . . . . . . . . . . . . . . . . 45

8.6 Model comparison - disturbance $4 \ldots \ldots \ldots \ldots \ldots$

8.7 Model comparison - disturbance 1 - magnified . . . . . . . . . . . . . . . . . . 47

8.8 Model comparison - disturbance 2 - magnified $\ldots \ldots \ldots \ldots$. . . . . . . . . 48

8.9 Model comparison - disturbance 4 - magnified . . . . . . . . . . . . . . . . . 49

8.10 Excitation system IEEE type AC4 … . . . . . . . . . . . . . . . . 51

8.11 SIME signal - disturbance 1 . . . . . . . . . . . . . . . . . . . . 52

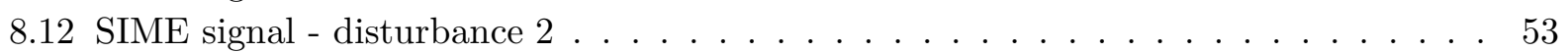

8.13 SIME signal - disturbance 1 - AVR $\ldots \ldots \ldots \ldots \ldots \ldots$. . . . . . . . . 54

8.14 SIME signal - disturbance 2 - AVR . . . . . . . . . . . . . . . . . . 55

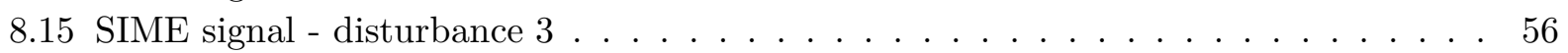

8.16 SIME signal - disturbance $4 \ldots \ldots \ldots \ldots \ldots \ldots$ 


\section{List of Tables}

6.1 Unknowns and load flow values . . . . . . . . . . . . . . . . . 34

8.1 Simulation time comparison - elapsed time in seconds . . . . . . . . . . . . . 50

A.1 Parameters of the governors . . . . . . . . . . . . . . . . . 63

A.2 Values of the parameters for the excitation svstem used in the model comparison . . 63

A.3 Parameter values for the excitation svstem in the SIME signal studv . . . . . . . . . 64

A.4 DFIG parameters . . . . . . . . . . . . . . . . . . . . . . 64

A.5 Values used in the control scheme for the model comparison . . . . . . . . . . . . . 64

A.6 Values used in the control scheme with SIME signal . . . . . . . . . . . . . . . . 64 


\section{Chapter 1}

\section{Introduction}

\subsection{Background}

The Kyoto protocol and the European Union's Climate Action and Renewable Energy Package have set down objectives for most countries. In this context, large investments in renewable energies are necessary for fulfilling the objectives. To help doing so, wind power is one of the most promising and most used technologies. In Europe, for instance, the total installed capacity was nearly $65 \mathrm{GW}$ at the end of 2008 , out of which almost $8.5 \mathrm{GW}$ was newly installed during 2008. This represents one third of the total newly installed capacity, all energy sources combined [1].

This means that the number of large wind parks are due to rise, which will seriously impact the existing power systems. These effects must be studied, using exhaustive models of wind turbines. However, due to the intricacy of the electric grid, models which are too detailed would take too long to simulate. Therefore a trade-off between simplicity and accuracy must be made, and so comprehensive models should be simplified.

\subsection{Aim}

The aim of this work is to use a specific software, Power Factory, to study the DFIG behavior. It will be seen in this work that Power Factory offers two built-in DFIG models. Another model is built, which enables us to get insights into creating models with this software. The three models are compared and one of them is used to study a specific control strategy.

\subsection{Different types of layout}

Wind turbines differ one from another to a great extent. Manufacturers have adopted different layouts, based mainly on choices upon the arrangement of mechanical and electrical components. We can mention, for instance, fixed- and variable-speed wind turbines. This is one of the most critical choices. A complete comparison between these two designs is outside the scope of this thesis. The reader interested in getting more details about the different layouts can be referred to [2].

\subsection{Different types of generator}

The generator models are one important part of the wind turbines' overall models. The two most common generators are the synchronous and induction (or asynchronous) generators. 
Synchronous generators offer higher efficiency and reactive power control capabilities, which are of high interest when thinking of controlling voltage to improve power system stability. They are however more expensive than induction generators. They are often decoupled from the grid by fully-rated converters, which add further to the overall cost, but enable them to run at a variable speed. Operating at variable speed allows the machine to adapt its speed to the wind speed in order to track the best operating point at which maximum power is produced.

Induction machines, running as motors, are well-known and widely used in the industry. They are sturdy, cheap and relied on mature technology. Nevertheless, they have seldom been used as generators before being employed in wind turbines. Among induction generators, doubly fed induction generators (DFIG) offer variable speed, while keeping the size of the controllers small so as to reduce the costs. In this thesis, we will compare different DFIG models.

\subsection{DigSilent Power Factory}

PowerFactory [3] is a software package made for power system simulations. It covers a wide range of simulations but will here be used for dynamic studies. As it will be seen, Power Factory offers two different models for induction generators. The first is a built-in component and the second comes as a built-in example in version 13. A new user-created model will be compared with the two built-in models.

\subsection{Layout of this work}

The thesis can be divided into three large parts. The first spans over chapters 2 to 4 . In these, the theory lying behind DFIG is explained. The reader familiar with machine theory can be referred directly to the second part. Chapters 5 to 7 present the models tested later and how they are controlled. Finally, results are given and commented in chapter 8 . 


\section{Chapter 2}

\section{Doubly fed induction generators}

In this chapter, we present doubly-fed induction generators. Relevant quantities are defined and a detailed model is given. The reader interested in delving deeper into the details of these generators can be referred to electric machinery and power system books such as [8, 9 . The review given in this section is largely inspired by these two references, as well as by [4.

\subsection{Working Principles}

An induction generator is composed by a stator and a rotor. In the case of a DFIG, both stator and rotor have three sinusoidally distributed windings, corresponding to three phases, displaced by $120^{\circ}$. The three phases are called $a, b$ and $c$. The stator has $p$ pairs of poles.

The rotor is connected to the grid through converters. A three-winding transformer gives different voltage levels for stator and rotor side. A schematic of such a system is presented in figure 2.1. When the machine produces energy, only a small part of the generated power flows from the rotor to the grid. The converters can then be chosen in accordance with this small rotor power. This means smaller converters compared to fully rated converters and this allows to decrease the costs.

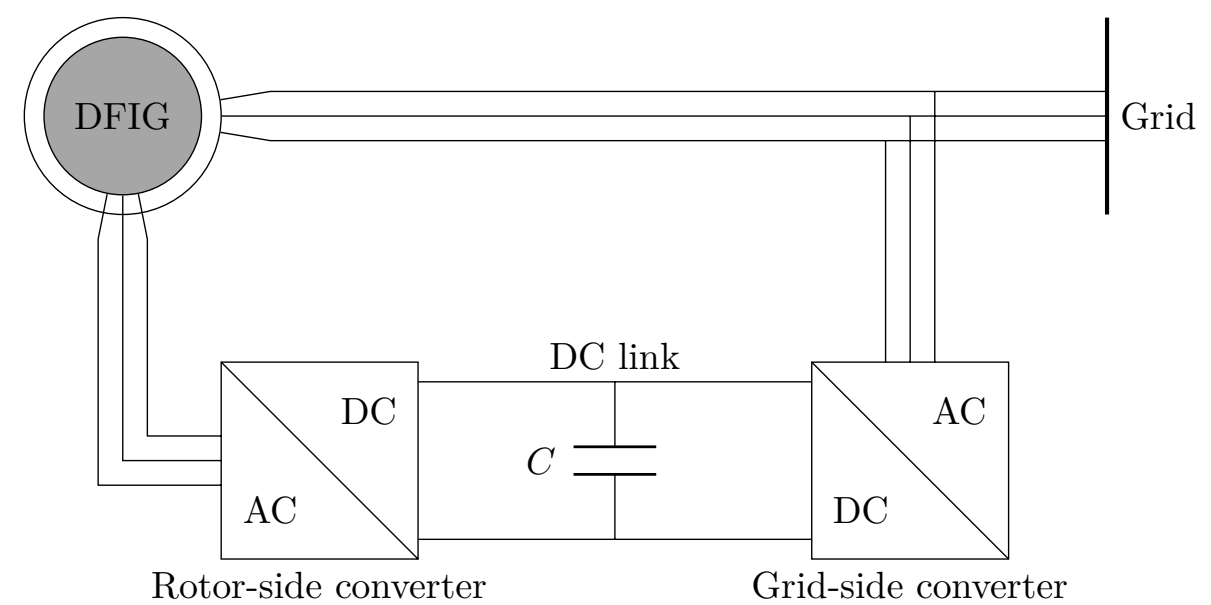

Figure 2.1: DFIG with its converters

The stator windings are connected to the grid which imposes the stator current frequency, 
$f_{s}$. The stator currents create a rotating magnetic field in the air gap. The rotational speed of this field, $\omega_{s}$, is proportional to $f_{s}$ :

$$
\omega_{s}=2 \pi f_{s} .
$$

If the rotor spins at a speed different from that of the rotational field, it sees a variation of magnetic flux. Therefore, by Faraday's law of induction, currents are induced in the rotor windings. Let us define $\omega_{m}$ the rotor mechanical speed and $\omega_{r}$ the rotor electrical speed by

$$
\omega_{r}=p \omega_{m}
$$

The flux linked by the rotor windings change with time if $\omega_{r} \neq \omega_{s}$. The machine operates usually as a generator if $\omega_{r}>\omega_{s}$ and as a motor otherwise. In the case of the DFIG however, it can operate in sub-synchronous mode as a generator [6]. The slip, $s$, defines the relative speed of the rotor compared with that of the stator:

$$
s=\frac{\omega_{s}-\omega_{r}}{\omega_{s}} .
$$

The slip is usually negative for a generator and positive for a motor. The currents induced in the rotor windings pulse at an angular speed defined by the difference between the synchronous speed and the rotor speed. Indeed, the stator currents at $\omega_{r}$ sees the rotating magnetic field created by the stator pulsating at $\omega_{s}-\omega_{r}$. It means the frequency of the rotor currents, $f_{r}$ is

$$
f_{r}=s f_{s} .
$$

If the rotor were to rotate at the synchronous speed, it would not see any change in magnetic fluxes. No currents would then be induced in its windings. Therefore, the machine operates always at speeds different from synchronous speed.

The rotor-side inverter controls the rotor currents. From (2.4), it can be noted that controlling the rotor currents controls the slip and so the speed of the machine.

\subsection{Modeling of doubly fed induction generators}

The following equations describe a three-phase symmetrical doubly fed induction generator.

\subsubsection{Electrical relations}

The voltage relations on rotor and stator sides are obtained by Kirchhoff's and Faraday's law:

$$
\begin{aligned}
& {\left[\begin{array}{l}
v_{a s} \\
v_{b s} \\
v_{c s}
\end{array}\right]=R_{s}\left[\begin{array}{l}
i_{a s} \\
i_{b s} \\
i_{c s}
\end{array}\right]+\frac{d}{d t}\left[\begin{array}{l}
\phi_{a s} \\
\phi_{b s} \\
\phi_{c s}
\end{array}\right],} \\
& {\left[\begin{array}{l}
v_{a r} \\
v_{b r} \\
v_{c r}
\end{array}\right]=R_{r}\left[\begin{array}{l}
i_{a r} \\
i_{b r} \\
i_{c r}
\end{array}\right]+\frac{d}{d t}\left[\begin{array}{l}
\phi_{a r} \\
\phi_{b r} \\
\phi_{c r}
\end{array}\right] .}
\end{aligned}
$$

The subscripts $r$ and $s$ denote rotor and stator quantities, respectively. The subscripts $a$, $b$ and $c$ are used for phases $a, b$ and $c$ quantities, respectively. The symbols $v$ and $i$ are for voltages and currents and $\phi$ represents flux linkages.

The stator and rotor winding resistances are $R_{s}$ and $R_{r}$. They are assumed to be equal for all phase windings. 
The flux linkages are coupled to the currents by the inductances:

$$
\begin{aligned}
& {\left[\begin{array}{l}
\phi_{a s} \\
\phi_{b s} \\
\phi_{c s}
\end{array}\right]=\boldsymbol{L}_{s}\left[\begin{array}{l}
i_{a s} \\
i_{b s} \\
i_{c s}
\end{array}\right]+\boldsymbol{L}_{m}\left[\begin{array}{c}
i_{a r} \\
i_{b r} \\
i_{c r}
\end{array}\right],} \\
& {\left[\begin{array}{l}
\phi_{a r} \\
\phi_{b r} \\
\phi_{c r}
\end{array}\right]=\boldsymbol{L}_{r}\left[\begin{array}{c}
i_{a r} \\
i_{b r} \\
i_{c r}
\end{array}\right]+\boldsymbol{L}_{m}^{T}\left[\begin{array}{c}
i_{a s} \\
i_{b s} \\
i_{c s}
\end{array}\right] .}
\end{aligned}
$$

The inductance matrices are defined by:

$$
\begin{aligned}
& \boldsymbol{L}_{s}= {\left[\begin{array}{ccc}
L_{l s}+L_{m} & -\frac{1}{2} L_{m} & -\frac{1}{2} L_{m} \\
-\frac{1}{2} L_{m} & L_{l s}+L_{m} & -\frac{1}{2} L_{m} \\
-\frac{1}{2} L_{m} & -\frac{1}{2} L_{m} & L_{l s}+L_{m}
\end{array}\right], } \\
& \boldsymbol{L}_{r}= {\left[\begin{array}{ccc}
L_{l r}+L_{m} & -\frac{1}{2} L_{m} & -\frac{1}{2} L_{m} \\
-\frac{1}{2} L_{m} & L_{l r}+L_{m} & -\frac{1}{2} L_{m} \\
-\frac{1}{2} L_{m} & -\frac{1}{2} L_{m} & L_{l r}+L_{m}
\end{array}\right], } \\
& \boldsymbol{L}_{m}=L_{m}\left[\begin{array}{ccc}
\cos \left(\theta_{r}\right) & \cos \left(\theta_{r}+\frac{2 \pi}{3}\right) & \cos \left(\theta_{r}-\frac{2 \pi}{3}\right) \\
\cos \left(\theta_{r}-\frac{2 \pi}{3}\right) & \cos \left(\theta_{r}\right) & \cos \left(\theta_{r}+\frac{2 \pi}{3}\right) \\
\cos \left(\theta_{r}+\frac{2 \pi}{3}\right) & \cos \left(\theta_{r}-\frac{2 \pi}{3}\right) & \cos \left(\theta_{r}\right)
\end{array}\right] .
\end{aligned}
$$

The subscripts $l$ and $m$ relate to the leakage and magnetizing inductances, respectively. The maximum amplitude of the mutual inductance between the stator and the rotor is $L_{m}$. The rotor electrical angular displacement regarding to the stator, defined from $\omega_{r}$, the electrical rotor speed is

$$
\theta_{r}(t)=\int_{0}^{t} \omega_{r} d t+\theta_{r}(0),
$$

where $\theta_{r}(0)$ is the initial position of the rotor at $\mathrm{t}=0$.

It can then be noted that the mutual inductance matrix $\boldsymbol{L}_{m}$ depends on time. In order to eliminate this time dependency, the $d q 0$-transformation will be used.

\subsubsection{Mechanical relations}

In the above section, the electrical dynamics of the DFIG have been developed in the stator reference frame. In order to complete the model, a model of the mechanical dynamics is here given. The dynamics of the generator shaft relate the rotor speed and the electromagnetic torque:

$$
J \frac{d \omega_{m}}{d t}=T_{m}-T_{e},
$$

where $J$ is the inertia of the machine, $T_{m}$ is the mechanical torque and $T_{e}$ is the electromagnetic torque.

\section{$2.3 d q 0$-reference frame}

In order to derive a simpler model, it is convenient to switch to a more suitable reference frame. One such reference frame is the so-called $d q 0$-reference frame.

The $d q 0$-reference frame is a rotating reference frame, defined by its displacement related to the stationary stator reference frame. Let $\beta_{d q}$ be the angular displacement between the d-axis 
and the rotor circuit. It is also defined by the angular speed $\omega_{d q}$ of the $d q$-reference frame relative to the stator:

$$
\beta_{d q}(t)=\int_{0}^{t} \omega_{d q} d t+\beta_{d q}(0),
$$

where $\beta_{d q}(0)$ is the initial angular displacement of the $d q$-axes relative to the stator circuit.

In this analysis, the $d q 0$-reference frame rotates at the synchronous speed $\omega_{s}$ in the stator stationary reference frame. Hence:

$$
\omega_{d q}=\omega_{s} .
$$

It means also that the $d q$-reference frame is displaced, relative to the rotor, by

$$
\beta_{r}=\beta_{d q}-\theta_{r}=\int_{0}^{t}\left(\omega_{s}-\omega_{r}\right) d t+\left(\beta_{d q}(0)-\theta_{r}(0)\right) .
$$

The new reference frame is represented in figure 2.2 together with the rotor reference frame rotating at $\omega_{r}$ and the stationary stator reference frame.

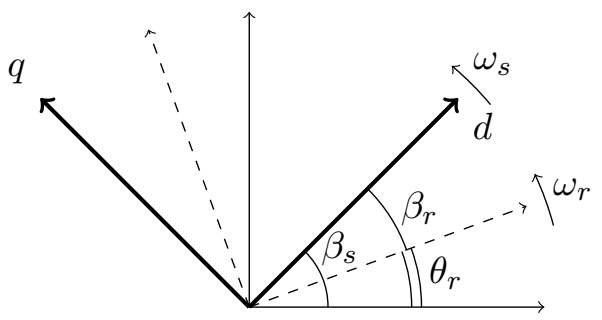

Figure 2.2: Relations between stator (thin), rotor (dashed) and synchronous (thick) reference frames

Let $\boldsymbol{T}_{d q 0}(\beta)$ be the rotation matrix which transforms the $a b c$-quantities into the $d q 0$-reference frame:

$$
\boldsymbol{T}_{d q 0}(\beta)=\sqrt{\frac{3}{2}}\left[\begin{array}{ccc}
\cos (\beta) & \cos \left(\beta-\frac{2 \pi}{3}\right) & \cos \left(\beta+\frac{2 \pi}{3}\right) \\
-\sin (\beta) & -\sin \left(\beta-\frac{2 \pi}{3}\right) & -\sin \left(\beta+\frac{2 \pi}{3}\right) \\
\frac{1}{\sqrt{2}} & \frac{1}{\sqrt{2}} & \frac{1}{\sqrt{2}}
\end{array}\right],
$$

where

- $\beta=\beta_{s}$ when the stator quantities are of interest,

- $\beta=\beta_{r}$ when the rotor quantities are of interest.

Then, the following relation holds between the $a b c$ - and $d q 0$-quantities:

$$
\vec{f}_{d q 0}=\boldsymbol{T}_{d q 0}(\beta) \vec{f}_{a b c},
$$

where:

$$
\begin{aligned}
& \vec{f}_{d q 0}=\left[\begin{array}{l}
f_{d} \\
f_{q} \\
f_{0}
\end{array}\right] \\
& \vec{f}_{a b c}=\left[\begin{array}{l}
f_{a} \\
f_{b} \\
f_{c}
\end{array}\right] .
\end{aligned}
$$


The notation $\vec{f}$ represents a vector quantity, which here may be a current, voltage or flux. It can be noted that for symmetrical $a b c$-quantities, the zero-component is zero. Indeed, the zero-component is, according to (2.17),

$$
f_{0}=\frac{\sqrt{3}}{2}\left(f_{a}+f_{b}+f_{c}\right)
$$

and, for symmetrical abc-quantities,

$$
f_{a}+f_{b}+f_{c}=0
$$

which leads directly to

$$
f_{0}=0
$$

The zero-component may therefore be omitted. Every quantity $\vec{f}_{d q 0}$ is therefore reduced to $\overrightarrow{f_{d q}}$.

\subsection{Modeling in $d q 0$-reference frame}

\subsubsection{Electrical equations}

Solving $\vec{f}_{a b c}$ in (2.18) for $\vec{f}$ being the stator and rotor voltages, currents and fluxes and substituting them into equations (2.5) , (2.6), (2.7) and (2.8), the following system of equations is obtained:

$$
\begin{aligned}
& \boldsymbol{T}_{d q 0}\left(\beta_{s}\right)^{-1} \vec{v}_{d q s}=R_{s} \boldsymbol{T}_{d q 0}\left(\beta_{s}\right)^{-1} \vec{\imath}_{d q s}+\frac{d}{d t}\left(\boldsymbol{T}_{d q 0}\left(\beta_{s}\right)^{-1} \vec{\phi}_{d q s}\right), \\
& \boldsymbol{T}_{d q 0}\left(\beta_{r}\right)^{-1} \vec{v}_{d q r}=R_{r} \boldsymbol{T}_{d q 0}\left(\beta_{r}\right)^{-1} \vec{\imath}_{d q r}+\frac{d}{d t}\left(\boldsymbol{T}_{d q 0}\left(\beta_{r}\right)^{-1} \vec{\phi}_{d q r}\right), \\
& \boldsymbol{T}_{d q 0}\left(\beta_{s}\right)^{-1} \vec{\phi}_{d q s}=\boldsymbol{L}_{s} \boldsymbol{T}_{d q 0}\left(\beta_{s}\right)^{-1} \vec{\imath}_{d q s}+\boldsymbol{L}_{m} \boldsymbol{T}_{d q 0}\left(\beta_{r}\right)^{-1} \vec{\imath}_{d q r}, \\
& \boldsymbol{T}_{d q 0}\left(\beta_{r}\right)^{-1} \vec{\phi}_{d q r}=\boldsymbol{L}_{r} \boldsymbol{T}_{d q 0}\left(\beta_{r}\right)^{-1} \vec{\imath}_{d q r}+\boldsymbol{L}_{m}^{T} \boldsymbol{T}_{d q 0}\left(\beta_{s}\right)^{-1} \vec{\imath}_{d q s} .
\end{aligned}
$$

Multiplying both sides of equations (2.24) and (2.26) by $\boldsymbol{T}_{d q 0}\left(\beta_{s}\right)$ and of equations (2.25) and (2.27) by $\boldsymbol{T}_{d q}\left(\beta_{r}\right)$, we get

$$
\begin{aligned}
\vec{v}_{d q s} & =R_{s} \vec{\imath}_{d q s}+\boldsymbol{T}_{d q 0}\left(\beta_{s}\right) \frac{d}{d t}\left(\boldsymbol{T}_{d q 0}\left(\beta_{s}\right)^{-1} \vec{\phi}_{d q s}\right), \\
\vec{v}_{d q r} & =R_{r} \vec{\imath}_{d q r}+\boldsymbol{T}_{d q 0}\left(\beta_{r}\right) \frac{d}{d t}\left(\boldsymbol{T}_{d q 0}\left(\beta_{r}\right)^{-1} \vec{\phi}_{d q r}\right) \\
\vec{\phi}_{d q s} & =\boldsymbol{T}_{d q 0}\left(\beta_{s}\right) \boldsymbol{L}_{s} \boldsymbol{T}_{d q 0}\left(\beta_{s}\right)^{-1} \vec{\imath}_{d q s}+\boldsymbol{T}_{d q 0}\left(\beta_{s}\right) \boldsymbol{L}_{m} \boldsymbol{T}_{d q 0}\left(\beta_{r}\right)^{-1} \vec{\imath}_{d q r}, \\
\vec{\phi}_{d q r} & =\boldsymbol{T}_{d q 0}\left(\beta_{r}\right) \boldsymbol{L}_{r} \boldsymbol{T}_{d q 0}\left(\beta_{r}\right)^{-1} \vec{\imath}_{d q r}+\boldsymbol{T}_{d q 0}\left(\beta_{r}\right) \boldsymbol{L}_{m}^{T} \boldsymbol{T}_{d q 0}\left(\beta_{s}\right)^{-1} \vec{\imath}_{d q s} .
\end{aligned}
$$

It is interesting to note that the last term on the right-hand side of the voltage equations gives rise to two terms:

$$
\boldsymbol{T}_{d q 0}\left(\beta_{s}\right) \frac{d}{d t}\left(\boldsymbol{T}_{d q 0}\left(\beta_{s}\right)^{-1} \vec{\phi}_{d q 0 s}\right)=\frac{d}{d t}\left[\begin{array}{c}
\phi_{d s} \\
\phi_{q s}
\end{array}\right]+\omega_{s}\left[\begin{array}{c}
-\phi_{q s} \\
\phi_{d s}
\end{array}\right]
$$


We get a corresponding equation for rotor voltages:

$$
\begin{aligned}
\boldsymbol{T}_{d q 0}\left(\beta_{r}\right) \frac{d}{d t}\left(\boldsymbol{T}_{d q 0}\left(\beta_{r}\right)^{-1} \vec{\phi}_{d q 0 r}\right) & =\frac{d}{d t}\left[\begin{array}{l}
\phi_{d r} \\
\phi_{q r}
\end{array}\right]+\left(\omega_{s}-\omega_{r}\right)\left[\begin{array}{c}
-\phi_{q r} \\
\phi_{d r}
\end{array}\right] \\
& =\frac{d}{d t}\left[\begin{array}{l}
\phi_{d r} \\
\phi_{q r}
\end{array}\right]+s \omega_{s}\left[\begin{array}{c}
-\phi_{q r} \\
\phi_{d r}
\end{array}\right],
\end{aligned}
$$

where $s$ is the slip defined in (2.3).

These two terms reflect the effect of switching from the stationary to the $d q 0$-reference frame. In particular, the very last term in the two equations above was not present in the original equation. It reflects the dependency on the new reference frame's angular speed. This term introduces also the cross-coupling between the $d$ - and $q$-components.

If the impedance matrices are now of interest, it appears that the transformation has made them diagonal, so that the flux equations become (without the zero-component):

$$
\begin{aligned}
& \vec{\phi}_{d q s}=\left[\begin{array}{cc}
L_{l s}+\frac{3}{2} L_{m} & 0 \\
0 & L_{l s}+\frac{3}{2} L_{m}
\end{array}\right] \vec{\imath}_{d q s}+\left[\begin{array}{cc}
\frac{3}{2} L_{m} & 0 \\
0 & \frac{3}{2} L_{m}
\end{array}\right] \vec{\imath}_{d q r}, \\
& \vec{\phi}_{d q r}=\left[\begin{array}{cc}
L_{l r}+\frac{3}{2} L_{m} & 0 \\
0 & L_{l r}+\frac{3}{2} L_{m}
\end{array}\right] \vec{\imath}_{d q 0 r}+\left[\begin{array}{cc}
\frac{3}{2} L_{m} & 0 \\
0 & \frac{3}{2} L_{m}
\end{array}\right] \vec{\imath}_{d q r} .
\end{aligned}
$$

Thus, equations (2.5), (2.6), (2.7) and (2.8) become, in the new reference frame:

$$
\begin{aligned}
& \vec{v}_{d q s}=\left[\begin{array}{l}
v_{d s} \\
v_{q s}
\end{array}\right]=R_{s}\left[\begin{array}{c}
i_{d s} \\
i_{q s}
\end{array}\right]+\frac{d}{d t}\left[\begin{array}{l}
\phi_{d s} \\
\phi_{q s}
\end{array}\right]+\omega_{s}\left[\begin{array}{c}
-\phi_{q s} \\
\phi_{d s}
\end{array}\right], \\
& \vec{v}_{d q r}=\left[\begin{array}{l}
v_{d r} \\
v_{q r}
\end{array}\right]=R_{r}\left[\begin{array}{c}
i_{d r} \\
i_{q r}
\end{array}\right]+\frac{d}{d t}\left[\begin{array}{c}
\phi_{d r} \\
\phi_{q r}
\end{array}\right]+s \omega_{s}\left[\begin{array}{c}
-\phi_{q r} \\
\phi_{d r}
\end{array}\right], \\
& \vec{\phi}_{d q s}=\left[\begin{array}{c}
\phi_{d s} \\
\phi_{q s}
\end{array}\right]=\left[\begin{array}{cc}
L_{l s}+\frac{3}{2} L_{m} & 0 \\
0 & L_{l s}+\frac{3}{2} L_{m}
\end{array}\right]\left[\begin{array}{c}
i_{d s} \\
i_{q s}
\end{array}\right]+\left[\begin{array}{cc}
\frac{3}{2} L_{m} & 0 \\
0 & \frac{3}{2} L_{m}
\end{array}\right]\left[\begin{array}{c}
i_{d r} \\
i_{q r}
\end{array}\right], \\
& \vec{\phi}_{d q r}=\left[\begin{array}{c}
\phi_{d r} \\
\phi_{q r}
\end{array}\right]=\left[\begin{array}{cc}
L_{l r}+\frac{3}{2} L_{m} & 0 \\
0 & L_{l r}+\frac{3}{2} L_{m}
\end{array}\right]\left[\begin{array}{c}
i_{d r} \\
i_{q r}
\end{array}\right]+\left[\begin{array}{cc}
\frac{3}{2} L_{m} & 0 \\
0 & \frac{3}{2} L_{m}
\end{array}\right]\left[\begin{array}{c}
i_{d s} \\
i_{q s}
\end{array}\right] .
\end{aligned}
$$

Several interesting comments can be made here.

First, the transformation has eliminated the time dependency of the mutual impedance matrix.

Furthermore, considering the flux linkage equations, the $d q$-components are magnetically decoupled, that is the flux linkages' $d$-component depends only on the currents' $d$-component, and a similar coupling exists between the $q$-components. It further reduces the complexity of the model.

Finally, it is worth noting that, with the matrix $\boldsymbol{T}_{d q 0}\left(\beta_{d q}\right)$ describing the transformation, the change of reference frame is power invariant.

An equivalent single-line diagram can be drawn for the machine as in figure 2.3 .

In this diagram and in the following, the generator convention is used by inverting all the currents.

In order to complete the electrical equations, the flux linkages' dependency on the synchronous speed appears by using the flux linkages per second. Reactances are used instead of inductances. The following notations are introduced: 


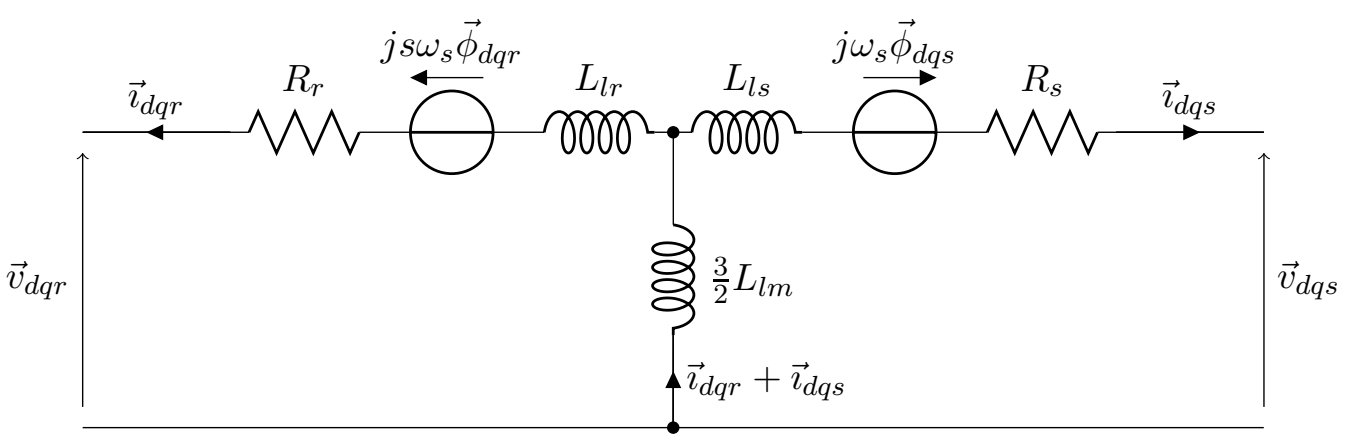

Figure 2.3: DFIG equivalent circuit

- flux linkages per second:

$$
\psi=\phi \omega_{s},
$$

- reactances $X$ :

$$
\begin{aligned}
X_{s} & =\omega_{s}\left(L_{l s}+\frac{3}{2} L_{m}\right), \\
X_{r} & =\omega_{s}\left(L_{l r}+\frac{3}{2} L_{m}\right), \\
X_{m} & =\frac{3}{2} \omega_{s} L_{m} .
\end{aligned}
$$

The electric equations therefore become:

$$
\begin{aligned}
& {\left[\begin{array}{l}
v_{d s} \\
v_{q s}
\end{array}\right]=-R_{s}\left[\begin{array}{c}
i_{d s} \\
i_{q s}
\end{array}\right]+\frac{1}{\omega_{s}} \frac{d}{d t}\left[\begin{array}{l}
\psi_{d s} \\
\psi_{q s}
\end{array}\right]+\left[\begin{array}{c}
-\psi_{q s} \\
\psi_{d s}
\end{array}\right],} \\
& {\left[\begin{array}{c}
v_{d r} \\
v_{q r}
\end{array}\right]=-R_{r}\left[\begin{array}{c}
i_{d r} \\
i_{q r}
\end{array}\right]+\frac{1}{\omega_{s}} \frac{d}{d t}\left[\begin{array}{l}
\psi_{d r} \\
\psi_{q r}
\end{array}\right]+s\left[\begin{array}{c}
-\psi_{q r} \\
\psi_{d r}
\end{array}\right],} \\
& {\left[\begin{array}{c}
\psi_{d s} \\
\psi_{q s}
\end{array}\right]=-\left[\begin{array}{c}
X_{s} i_{d s}+X_{m} i_{d r} \\
X_{s} i_{q s}+X_{m} i_{q r}
\end{array}\right]} \\
& {\left[\begin{array}{c}
\psi_{d r} \\
\psi_{q r}
\end{array}\right]=-\left[\begin{array}{c}
X_{r} i_{d r}+X_{m} i_{d s} \\
X_{r} i_{q r}+X_{m} i_{q s}
\end{array}\right]}
\end{aligned}
$$

\subsubsection{Phasor notation}

The vector notation can be heavy to manipulate. Therefore, drawing a similarity to the complex notation, the following expression can be adopted:

$$
\bar{f}_{d q}=f_{d}+j f_{q} \Leftrightarrow \vec{f}_{d q}=\left[\begin{array}{l}
f_{d} \\
f_{q}
\end{array}\right] .
$$

This allows us to manipulate the quantities with much more flexibility without using the cumbersome vector notation. The quantity $j \bar{f}_{d q}$ is, for instance,

$$
j \bar{f}_{d q}=j\left(f_{d}+j f_{q}\right)=-f_{q}+j f_{d} \Leftrightarrow\left[\begin{array}{c}
-f_{q} \\
f_{d}
\end{array}\right] .
$$


This is of interest in this analysis, since such quantities appear in the last term of the right-hand side of the voltage equations. The electrical relations become

$$
\begin{aligned}
& \bar{v}_{d q s}=-\bar{R}_{s} i_{d q s}+\frac{1}{\omega_{s}} \frac{d \bar{\psi}_{d q s}}{d t}+j \bar{\psi}_{d q s} \\
& \bar{v}_{d q r}=-\bar{R}_{r} i_{d q r}+\frac{1}{\omega_{s}} \frac{d \bar{\psi}_{d q r}}{d t}+j s \bar{\psi}_{d q r} \\
& \bar{\psi}_{d q s}=-X_{s} \bar{i}_{d q s}-X_{m} \bar{i}_{d q r} \\
& \bar{\psi}_{d q r}=-X_{r} \bar{i}_{d q r}-X_{m} \bar{i}_{d q s} .
\end{aligned}
$$

\subsubsection{Mechanical equations}

Both mechanical and electromagnetic torques can be expressed as functions of power.

The mechanical torque $T_{m}$ is related to the mechanical power $P_{m}$ extracted from the wind and available on the turbine's shaft:

$$
T_{m}=\frac{P_{m}}{\omega_{m}}=\frac{p}{\omega_{r}} P_{m} .
$$

The electromagnetic torque can be derived from a power balance [4. It can be expressed as a function of $P_{t}$, the power available on the shaft of the generator by

$$
T_{e}=\frac{P_{t}}{\omega_{m}}
$$

In the induction machine, the power transmitted by the wind turbine to the generator is lost in ohmic losses $P_{\text {loss }}$, used for magnetizing the machine $P_{\text {mag }}$ or available as generated power $P_{g}$. This means that the transmitted power can be expressed as

$$
P_{t}=P_{l o s s}+P_{m a g}+P_{g}
$$

The generated power is the active power supplied by the stator and the rotor, expressed in the $d q 0$-reference frame by

$$
P_{g}=\vec{v}_{d q s}^{T} \vec{i}_{d q s}+\vec{v}_{d q r}^{T} \vec{i}_{d q r}=\left(v_{s d} i_{d s}+v_{s q} i_{q s}\right)+\left(v_{r d} i_{d r}+v_{r q} i_{q r}\right) .
$$

Substituting the rotor and stator voltages by their expressions from (2.44) and (2.45), we get

$$
\begin{aligned}
P_{g} & =-\underbrace{R_{s}\left(i_{d s}^{2}+i_{q s}^{2}\right)}_{\text {Stator ohmic losses }}-\underbrace{R_{r}\left(i_{d r}^{2}+i_{q r}^{2}\right)}_{\text {Rotor ohmic losses }} \\
& -\underbrace{\left(-\frac{1}{\omega_{s}}\left(\frac{d \psi_{d s}}{d t} i_{d s}+\frac{d \psi_{q s}}{d t} i_{q s}+\frac{d \psi_{d r}}{d t} i_{d r}+\frac{d \psi_{q r}}{d t} i_{q r}\right)\right)}_{\text {Magnetizing power }} \\
& +\frac{\omega_{d q}}{\omega_{s}}\left(\psi_{d s} i_{q s}-\psi_{q s} i_{d s}\right)+\frac{\omega_{d q}-\omega_{r}}{\omega_{s}}\left(\psi_{d r} i_{q r}-\psi_{q r} i_{d r}\right) .
\end{aligned}
$$

Hence, the ohmic losses and the magnetizing power appear in the expression of the generated power. The transmitted power is then

$$
P_{t}=\frac{\omega_{d q}}{\omega_{s}}\left(\psi_{d s} i_{q s}-\psi_{q s} i_{d s}\right)+\frac{\omega_{d q}-\omega_{r}}{\omega_{s}}\left(\psi_{d r} i_{q r}-\psi_{q r} i_{d r}\right) .
$$


This expression is valid regardless the speed of the $d q 0$-reference frame, $\omega_{d q}$. A simple expression of $P_{t}$ is obtained by setting either $\omega_{d q}=0$ (the $d q 0$-reference frame is then simply the stator stationary reference frame) or $\omega_{d q}=\omega_{r}$ (the $d q 0$-reference frame rotates with the stator).

$$
\begin{gathered}
\left(\omega_{d q}=0\right) \Rightarrow\left\{\begin{array}{l}
P_{t}=\frac{\omega_{r}}{\omega_{s}}\left(\psi_{q r} i_{d r}-\psi_{d r} i_{q r}\right) . \\
T_{e}=\frac{p}{\omega_{r}} P_{t}=\frac{p}{\omega_{s}}\left(\psi_{q r} i_{d r}-\psi_{d r} i_{q r}\right) .
\end{array}\right. \\
\left(\omega_{d q}=\omega_{r}\right) \Rightarrow\left\{\begin{array}{l}
P_{t}=\frac{\omega_{r}}{\omega_{s}}\left(\psi_{d s} i_{q s}-\psi_{q s} i_{d s}\right) . \\
T_{e}=\frac{p}{\omega_{r}} P_{t}=\frac{p}{\omega_{s}}\left(\psi_{d s} i_{q s}-\psi_{q s} i_{d s}\right) .
\end{array}\right.
\end{gathered}
$$

Introducing

$$
P_{e}=\psi_{q r} i_{d r}-\psi_{d r} i_{q r}=\psi_{d s} i_{q s}-\psi_{q s} i_{d s}
$$

the electromagnetic torque can be written as

$$
T_{e}=\frac{p}{\omega_{s}} P_{e} .
$$

\subsection{Per-unit System}

The per-unit system allows work with normalized values. It is especially useful when a power system of interest is composed of different voltage levels, which are separated by transformers. All quantities can then be dealt with independently at the different voltage levels, which simplifies the analysis. Each per-unit quantity $f^{p u}$ is obtained by dividing the corresponding value $f$ by the base quantity $f_{\text {base }}$ :

$$
f^{p u}=\frac{f}{f_{\text {base }}} .
$$

Usually, base voltage and power are defined and the other per-unit quantities are derived from these two values. Base voltages $V_{\text {base }}$ regarding each voltage level is here defined as the phaseto-neutral voltage of the corresponding zone. The base power $S_{\text {base }}$ is chosen considering, for example, the rated power of the machine $S_{n}$. The following relation then holds (keeping in mind that the base voltage is defined as a phase-to-neutral voltage):

$$
S_{\text {base }}=3 V_{\text {base }} I_{\text {base }},
$$

which allows to define the base current $I_{\text {base }}$.

Next, the base impedance $Z_{\text {base }}$ is defined by

$$
Z_{\text {base }}=\frac{V_{\text {base }}}{I_{\text {base }}}=\frac{S_{\text {base }}}{3 I_{\text {base }}^{2}}=\frac{V_{\text {base }}^{2}}{S_{\text {base }}} .
$$

The flux linkages per second has the dimension of a voltage and their base value is therefore the base voltage. Electrical relations (2.44), (2.45), (2.46) and (2.47) can be rewritten by dividing 
both sides by $V_{\text {base }}=Z_{\text {base }} I_{\text {base }}$ :

$$
\begin{aligned}
& \frac{1}{V_{\text {base }}} \bar{v}_{d q s}=-\frac{R_{s}}{Z_{\text {base }}} \frac{\bar{l}_{d q s}}{I_{\text {base }}}+\frac{1}{\omega_{s}} \frac{1}{V_{\text {base }}} \frac{d \bar{\psi}_{d q s}}{d t}+j \frac{\bar{\psi}_{d q s}}{V_{\text {base }}}, \\
& \frac{1}{V_{\text {base }}} \bar{v}_{d q r}=-\frac{R_{r}}{Z_{\text {base }}} \frac{\bar{l}_{d q r}}{I_{\text {base }}}+\frac{1}{\omega_{s}} \frac{1}{V_{\text {base }}} \frac{d \bar{\psi}_{d q r}}{d t}+j \frac{\omega_{s}-\omega_{r}}{\omega_{s}} \frac{\bar{\psi}_{d q r}}{V_{\text {base }}}, \\
& \frac{1}{V_{\text {base }}} \bar{\psi}_{d q s}=-\frac{X_{s}}{Z_{\text {base }}} \frac{\bar{l}_{d q s}}{I_{\text {base }}}-\frac{X_{m}}{Z_{\text {base }}} \frac{\bar{n}_{d q r}}{I_{\text {base }}}, \\
& \frac{1}{V_{\text {base }}} \bar{\psi}_{d q r}=-\frac{X_{r}}{Z_{\text {base }}} \frac{\bar{l}_{d q r}}{I_{\text {base }}}-\frac{X_{m}}{Z_{\text {base }}} \frac{\bar{l}_{d q s}}{I_{\text {base }}},
\end{aligned}
$$

which means that the quantities in per-unit naturally appears, without supplementary coefficient.

The program used in this work, Power Factory, uses a specific per-unit system for the mechanical equation, based on the following quantities:

- $s_{n}$ : nominal slip,

- $P_{n m}$ : Rated mechanical power.

These quantities are specific to each DFIG. From these quantities, a base torque can be defined:

$$
T_{\text {base }}=\frac{P_{n m}}{\left(1-s_{n}\right) \frac{\omega_{s}}{p}}
$$

Power Factory uses per-unit speeds, with the base speed defined as

$$
\omega_{\text {base }}=\frac{\omega_{s}}{p}
$$

The mechanical equation 2.13 can now be rewritten per-unit:

$$
J \omega_{\text {base }} \frac{d \omega_{m}^{p u}}{d t}=T_{\text {base }}\left(T_{m}^{p u}-T_{e}^{p u}\right) .
$$

The so-called acceleration time constant is then defined as

$$
T_{\text {ag }}=\frac{J \omega_{\text {base }}}{T_{\text {base }}} .
$$

The per-unit mechanical equation becomes

$$
\frac{d \omega_{m}^{p u}}{d t}=\frac{1}{T_{\mathrm{ag}}}\left(T_{m}^{p u}-T_{e}^{p u}\right),
$$

with

$$
\begin{aligned}
T_{m}^{p u} & =\frac{1}{T_{\text {base }}} \frac{P_{m}}{\omega_{\text {base }} \omega_{m}^{p u}} \\
T_{e}^{p u} & =\frac{1}{T_{\text {base }}} \frac{P_{e}}{\omega_{\text {base }}}
\end{aligned}
$$

From here on, the superscript $p u$ in $f^{\bar{p} u}$ will be dropped and all the quantities are expressed in per-unit if it is not said otherwise. 
Five equations constitute the detailed model:

$$
\begin{aligned}
\bar{v}_{d q s} & =-R_{s} \bar{i}_{d q s}+\frac{1}{\omega_{s}} \frac{d \bar{\psi}_{d q s}}{d t}+j \bar{\psi}_{d q s} \\
\bar{v}_{d q r} & =-R_{r} \bar{i}_{d q r}+\frac{1}{\omega_{s}} \frac{d \bar{\psi}_{d q r}}{d t}+j s \bar{\psi}_{d q r} \\
\bar{\psi}_{d q s} & =-X_{s} \bar{i}_{d q s}-X_{m} \bar{i}_{d q r} \\
\bar{\psi}_{d q r} & =-X_{r} \bar{i}_{d q r}-X_{m} \bar{i}_{d q s} \\
\frac{d \omega_{m}}{d t} & =\frac{1}{T_{\mathrm{ag}}}\left(T_{m}-T_{e}\right) .
\end{aligned}
$$

A total of five differential equations must be solved, simultaneously. Based on reasonable assumptions, reduced-order models will be derived. The design of controllers based on these model is simplified. 



\section{Chapter 3}

\section{Reduced order models}

\subsection{Third order model}

\subsubsection{Neglecting the stator transients}

When doing the simulations, the grid transients are usually neglected. They correspond to fast terms varying with a high frequency which is not of interest for studying electromechanical transients. Furthermore, including these high-frequency terms in the computations would require small integration steps to capture their dynamics [9]. Therefore, the grid model is simplified by neglecting the variations of these terms. Keeping these terms in the DFIG model would be inconsistent and it would make the model unnecessarily complicated. Neglecting these fast varying terms is equivalent to neglect the stator transients. In the $d q 0$-reference frame rotating at synchronous speed, it means neglecting the $\frac{d \psi_{s}}{d t}$ term in the stator electrical equation [7]. It eliminates two differential equations of the model which then becomes a so-called third-order model. The stator electrical equation becomes

$$
\bar{v}_{d q s}=-R_{s} \bar{v}_{d q s}+j \bar{\psi}_{d q s} .
$$

From equations (2.80) and (2.81), the stator flux can be expressed in terms of stator currents and rotor fluxes as

$$
\bar{\psi}_{d q s}=\frac{X_{m}}{X_{r}} \bar{\psi}_{d q r}-\left(X_{s}-\frac{X_{m}^{2}}{X_{r}} \bar{\imath}_{d q s}\right) .
$$

Substituting in (3.1):

$$
\bar{v}_{d q s}=-\left(R_{s}+j\left(X_{s}-\frac{X_{m}^{2}}{X_{r}}\right)\right) \bar{\imath}_{d q s}+j \frac{X_{m}}{X_{r}} \bar{\psi}_{d q r},
$$

and defining

$$
\begin{aligned}
& \bar{E}^{\prime}=j \frac{X_{m}}{X_{r}} \bar{\psi}_{d q r} \\
& Z^{\prime}=R_{s}+j\left(X_{s}-\frac{X_{m}^{2}}{X_{r}}\right)=R_{s}+j X^{\prime},
\end{aligned}
$$

with:

$$
X^{\prime}=\left(X_{s}-\frac{X_{m}^{2}}{X_{r}}\right)
$$

the voltage equation can be rewritten

$$
\bar{v}_{d q s}=\bar{E}^{\prime}-Z^{\prime} \bar{\imath}_{d q s}
$$


The stator can be represented in a very simple way by a voltage source $\bar{E}^{\prime}$ behind an impedance $Z^{\prime}$, see figure 3.1 .

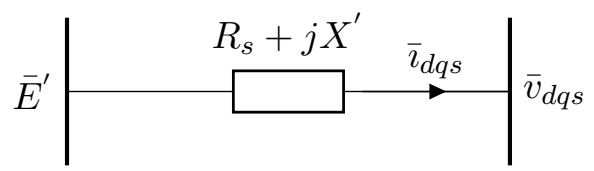

Figure 3.1: Stator equivalent circuit with stator flux transients neglected

\subsubsection{State space formulation}

The system can now be described by three state variables only, which can be chosen to be the two components of $\bar{E}^{\prime}, E_{d}^{\prime}$ and $E_{q}^{\prime}$, and $\omega_{r}$. Expressing rotor currents in terms of rotor fluxes and stator currents from (2.81):

$$
\bar{\imath}_{d q r}=-\frac{1}{X_{r}}\left(\bar{\psi}_{d q r}+X_{m} \bar{\imath}_{d q s}\right),
$$

substituting rotor currents by this expression in the rotor voltage equation (2.79):

$$
\bar{v}_{d q r}=R_{r}\left(\frac{1}{X_{r}}\left(\bar{\psi}_{d q r}+X_{m} \bar{\imath}_{d q s}\right)\right)+\frac{1}{\omega_{s}} \frac{d \bar{\psi}_{d q r}}{d t}+j s \bar{\psi}_{d q r},
$$

and introducing $\bar{E}^{\prime}$ by substituting $\bar{\psi}_{d q r}$ from (3.4), and solving the rotor voltage equation above for $\frac{d \bar{E}^{\prime}}{d t}$, we get

$$
\frac{d \bar{E}^{\prime}}{d t}=-\omega_{s} \frac{R_{r}}{X_{r}} \bar{E}^{\prime}-s \omega_{s}\left(j \bar{E}^{\prime}\right)-j \omega_{s} \frac{R_{r}}{X_{r}} \frac{X_{m}^{2}}{X_{r}} \bar{l}_{d q s}+j \omega_{s} \frac{X_{m}}{X_{r}} \bar{v}_{d q r}
$$

Introducing the so-called transient open-circuit time constant $T_{0}$ :

$$
T_{0}=\frac{X_{r}}{\omega_{s} R_{r}}
$$

and the new notation $X^{\prime}(3.5)$ leads to:

$$
\frac{d \bar{E}^{\prime}}{d t}=\frac{1}{T_{0}}\left(-\bar{E}^{\prime}-s \omega_{s} T_{0}\left(j \bar{E}^{\prime}\right)-j\left(X_{s}-X^{\prime}\right) \bar{\imath}_{d q s}+j T_{0} \omega_{s} \frac{X_{m}}{X_{r}} \bar{v}_{d q r}\right) .
$$

Expressing the stator currents now in terms of the input $\bar{v}_{d q s}$ and state variable $\bar{E}^{\prime}$ from (3.7):

$$
\bar{\imath}_{d q s}=\frac{\bar{E}^{\prime}-\bar{v}_{d q s}}{Z^{\prime}}
$$

and substituting in (3.12), we get

$$
\frac{d \bar{E}^{\prime}}{d t}=\frac{1}{T_{0}}\left(-\bar{E}^{\prime}-s \omega_{s} T_{0}\left(j \bar{E}^{\prime}\right)-j\left(X_{s}-X^{\prime}\right) \frac{\bar{E}^{\prime}-\bar{v}_{d q s}}{Z^{\prime}}+j T_{0} \omega_{s} \frac{X_{m}}{X_{r}} \bar{v}_{d q r}\right) .
$$

Reorganizing the terms, substituting $Z^{\prime}$ by its expression from (3.5) and using

$$
\frac{1}{Z^{\prime}}=\frac{\left(R_{s}-j X^{\prime}\right)}{\left|Z^{\prime}\right|^{2}}
$$


the following is obtained:

$$
\begin{aligned}
\frac{d \bar{E}^{\prime}}{d t}= & \frac{1}{T_{0}}\left(-\left(1+X^{\prime} \frac{X_{s}-X^{\prime}}{\left|Z^{\prime}\right|^{2}}\right) \bar{E}^{\prime}-\left(s \omega_{s} T_{0}+R_{s} \frac{X_{s}-X^{\prime}}{\left|Z^{\prime}\right|^{2}}\right)\left(j \bar{E}^{\prime}\right)\right) \\
& +\frac{1}{T_{0}}\left(X^{\prime} \frac{X_{s}-X^{\prime}}{\left|Z^{\prime}\right|^{2}} \bar{v}_{d q s}+R_{s} \frac{X_{s}-X^{\prime}}{\left|Z^{\prime}\right|^{2}}\left(j \bar{v}_{d q s}\right)+j T_{0} \omega_{s} \frac{X_{m}}{X_{r}} \bar{v}_{d q r}\right) .
\end{aligned}
$$

This equation pictures the dynamics of the state variables $E_{d}^{\prime}$ and $E_{q}^{\prime}$ with respect to the inputs $\bar{v}_{d q s}$ and $\bar{v}_{d q r}$. It can be expressed in the state space form as

$$
\begin{aligned}
{\left[\begin{array}{c}
\frac{d E_{d}^{\prime}}{d t} \\
\frac{d E_{q}^{\prime}}{d t}
\end{array}\right]=} & \frac{1}{T_{0}}\left[\begin{array}{cc}
-\left(1+X^{\prime} \frac{X_{s}-X^{\prime}}{\left|Z^{\prime}\right|^{2}}\right) & s \omega_{s} T_{0}+R_{s} \frac{X_{s}-X^{\prime}}{\left|Z^{\prime}\right|^{2}} \\
-\left(s \omega_{s} T_{0}+R_{s} \frac{X_{s}-X^{\prime}}{\left|Z^{\prime}\right|^{2}}\right) & -\left(1+X^{\prime} \frac{X_{s}-X^{\prime}}{\left|Z^{\prime}\right|^{2}}\right)
\end{array}\right]\left[\begin{array}{c}
E_{d}^{\prime} \\
E_{q}^{\prime}
\end{array}\right] \\
& +\frac{1}{T_{0}}\left[\begin{array}{cccc}
X^{\prime} \frac{X_{s}-X^{\prime}}{\left|Z^{\prime}\right|^{2}} & -R_{s} \frac{X_{s}-X^{\prime}}{\left|Z^{\prime}\right|^{2}} & 0 & -T_{0} \omega_{s} \frac{X_{m}}{X_{r}} \\
R_{s} \frac{X_{s}-X^{\prime}}{\left|Z^{\prime}\right|^{2}} & X^{\prime} \frac{X_{s}-X^{\prime}}{\left|Z^{\prime}\right|^{2}} & T_{0} \omega_{s} \frac{X_{m}}{X_{r}} & 0
\end{array}\right]\left[\begin{array}{c}
v_{d s} \\
v_{q s} \\
v_{d r} \\
v_{q r}
\end{array}\right]
\end{aligned}
$$

\subsubsection{Neglecting the stator resistance}

Another common assumption to further simplify the third-order model is to neglect the stator resistance which is often assumed to be very small. The stator voltage equation is simplified to

$$
\bar{v}_{d q s}=j \bar{\psi}_{d q s} .
$$

The stator can be represented as a voltage $\bar{E}^{\prime}$ behind a reactance $X^{\prime}$ as in figure 3.2 .

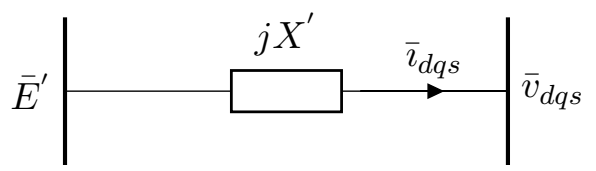

Figure 3.2: Stator equivalent circuit with rotor resistance neglected

Equation (3.16) becomes

$$
\frac{d \bar{E}^{\prime}}{d t}=\frac{1}{T_{0}}\left(-\frac{X_{s}}{X^{\prime}} \bar{E}^{\prime}-s \omega_{s} T_{0}\left(j \bar{E}^{\prime}\right)+\frac{X_{s}-X^{\prime}}{X^{\prime}} \bar{v}_{d q s}+j T_{0} \omega_{s} \frac{X_{m}}{X_{r}} \bar{v}_{d q r}\right) .
$$

Rewriting this in state space form:

$$
\begin{aligned}
{\left[\begin{array}{c}
\frac{d E_{d}^{\prime}}{d t^{\prime}} \\
\frac{d E_{q}^{\prime}}{d t}
\end{array}\right]=} & \frac{1}{T_{0}}\left[\begin{array}{cc}
-\frac{X_{s}}{X^{\prime}} & s \omega_{s} T_{0} \\
-s \omega_{s} T_{0} & -\frac{X_{s}}{X^{\prime}}
\end{array}\right]\left[\begin{array}{c}
E_{d}^{\prime} \\
E_{q}^{\prime}
\end{array}\right] \\
& +\frac{1}{T_{0}}\left[\begin{array}{cccc}
\frac{X_{s}-X^{\prime}}{X^{\prime}} & 0 & 0 & -T_{0} \omega_{s} \frac{X_{m}}{X_{r}} \\
0 & \frac{X_{s}-X^{\prime}}{X^{\prime}} & T_{0} \omega_{s} \frac{X_{m}}{X_{r}} & 0
\end{array}\right]\left[\begin{array}{c}
v_{d s} \\
v_{q s} \\
v_{d r} \\
v_{q r}
\end{array}\right]
\end{aligned}
$$

Here the state variables have been chosen to be $E_{d}$ and $E_{q}$. This choice is convenient to compare DFIGs with synchronous generators. 


\subsubsection{Power considerations}

Thanks to both assumptions, some insights regarding the generating power are gained. Recalling the definition of $P_{e}$ in (2.62) and the simplified voltage equation (3.18), the electrical power can now be expressed as

$$
P_{e}=v_{d s} i_{d s}+v_{q s} i_{q s}=\operatorname{Re}\left(\bar{v}_{d q s} \bar{\imath}_{d q s}\right) P_{s} .
$$

It means that the power flowing out of the stator is now directly linked to the electric torque (2.77). Secondly, the transmitted power can also be written with respect to the stator power. From equation (2.59) in the $d q 0$-reference frame rotating at synchronous speed:

$$
P_{t}=(1-s) P_{s} .
$$

The power produced from the stator is then a simple function of the slip and the transmitted power. The total generated power, $P_{g}$ in (2.57), is the sum of the power flowing from the stator, $P_{s}$, and the power flowing out of the rotor, $P_{r}$. Considering the losses and the power demand for magnetizing the machine, this generated power is smaller than the transmitted power, that is

$$
P_{s}+P_{r}<P_{t} .
$$

Given the above relation between the transmitted power and the stator power, the following limit for rotor power holds:

$$
P_{r}<-s P_{s}
$$

The power produced from the rotor then accounts for a small part of the total power. This is an important feature of the DFIG which is controlled on the rotor side. The controllers can then be designed to only handle a small fraction of the total generated power. This helps to keep the costs as low as possible.

\subsubsection{Mechanical equation}

The mechanical dynamics are now a function of the stator power, since the electrical power and stator power are equal:

$$
\frac{d \omega_{r}}{d t}=\frac{1}{M}\left(\frac{\omega_{s}}{\omega_{r}} P_{m}-P_{s}\right) .
$$

It allows to design a controller which can act upon the rotor speed through the stator power. It will be seen in section 5 that the stator active power can be controlled by rotor currents.

\subsection{First order model}

The third order model may be reduced even more by neglecting the rotor transients [10, 11]. The voltage equations become algebraic:

$$
\begin{aligned}
& \bar{v}_{d q s}=-\left(R_{s}+j X_{s}\right) \bar{\imath}_{d q s}-j X_{m} \bar{\imath}_{d q r}, \\
& \bar{v}_{d q r}=-\left(R_{r}+s j X_{r}\right) \bar{\imath}_{d q r}-s j X_{m} \bar{\imath}_{d q s} .
\end{aligned}
$$

The only dynamics left are the mechanical dynamics, described by equation (3.25). The fictitious voltage $\bar{E}^{\prime}$ can then be directly computed from the stator and rotor voltages. We can solve equation (3.20) for the two components of $\bar{E}^{\prime}$ :

$$
\left[\begin{array}{c}
E_{d}^{\prime} \\
E_{q}^{\prime}
\end{array}\right]=\frac{1}{\frac{X_{s}^{2}}{X^{\prime 2}}+\left(s \omega_{s} T_{0}\right)^{2}}\left[\begin{array}{cc}
-\frac{X_{s}}{X^{\prime}} & -s \omega_{s} T_{0} \\
s \omega_{s} T_{0} & -\frac{X_{s}}{X^{\prime}}
\end{array}\right]\left[\begin{array}{c}
-\frac{X_{s}-X^{\prime}}{X^{\prime}} V_{d s}+\omega_{s} X_{m} \frac{T_{0}}{X_{r}} V_{q r} \\
-\frac{X_{s}-X^{\prime}}{X^{\prime}} V_{q s}-\omega_{s} X_{m} \frac{T_{0}}{X_{r}} V_{d r}
\end{array}\right] .
$$




\section{Chapter 4}

\section{Converter models}

The DFIG is generally used with two back-to-back PWM converters as shown in figure 2.1. The grid-side controller is used to keep the DC-link voltage constant and to make the grid side run at unity power factor, so that no reactive power is flowing between the rotor and the grid. The rotor-side controller allows to control stator voltage and stator active power [6].

\subsection{Supply-side converter}

The supply side can be modelled as in figure 4.1.

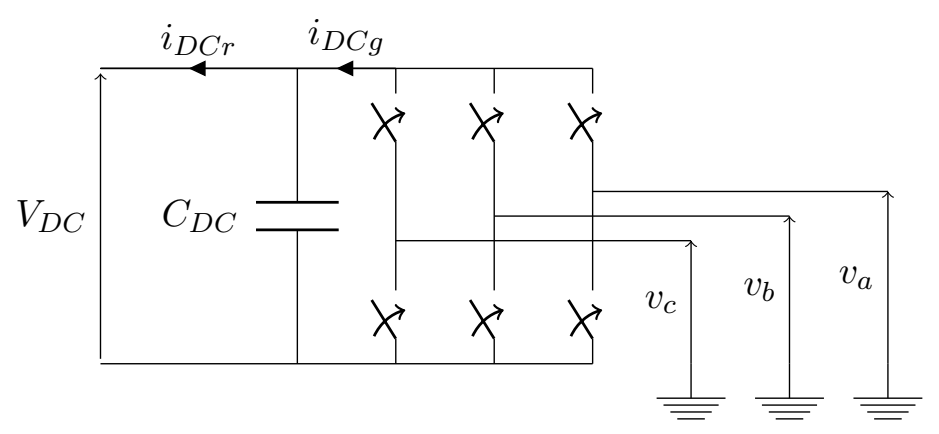

Figure 4.1: Grid-side model

The grid-side converter is supplied with three phase-voltages coming from the grid. Assuming a symmetrical grid, voltages $v_{a}, v_{b}$ and $v_{c}$ can be written as

$$
\begin{aligned}
& v_{a}=V_{s} \sin \left(\omega_{g} t+\phi_{g}\right) \\
& v_{b}=V_{s} \sin \left(\omega_{g} t+\frac{2 \pi}{3}+\phi_{g}\right) \\
& v_{a}=V_{s} \sin \left(\omega_{g} t-\frac{2 \pi}{3}+\phi_{g}\right),
\end{aligned}
$$

where $\phi_{g}$ is the phase of voltage $v_{a}, \omega_{g}$ is the electric frequency of the grid and $V_{s}$ is the RMS stator voltage.

The PWM converter switches the transistors on and off according to a control signal it receives from the controller. The controller delivers the PWM modulation depth, $P_{m 1}$, as a control signal. The modulation depth relates the grid voltage magnitude $V_{s}$ to the dc-voltage 
$V_{D C}:$

$$
V_{s}=P_{m 1} \frac{\sqrt{3} V_{D C}}{2 \sqrt{2}} .
$$

\subsection{Rotor-side converter}

The rotor side can be modelled as in figure 4.2.

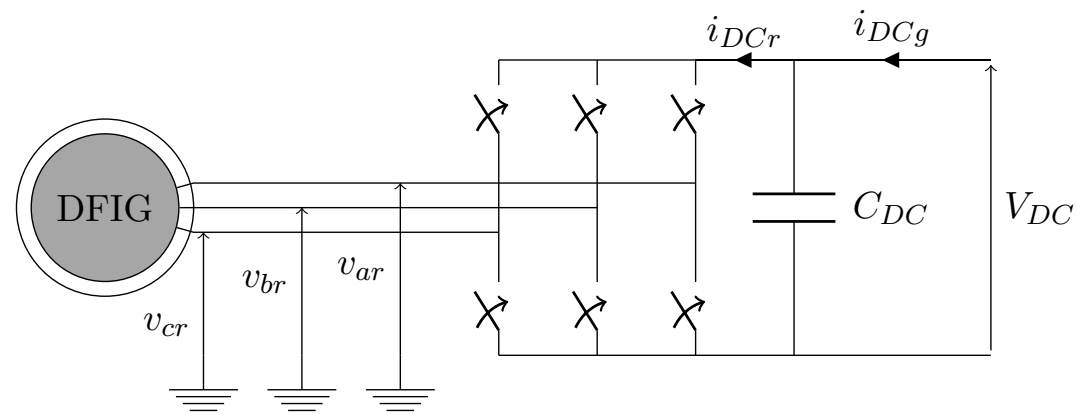

Figure 4.2: Rotor-side model

The quantities $v_{a r}, v_{b r}$ and $v_{c r}$ are the three rotor phase-voltages defined by

$$
\begin{aligned}
& v_{a r}=V_{r} \sin \left(\omega_{r} t+\phi_{g r}\right) \\
& v_{b r}=V_{r} \sin \left(\omega_{r} t+\frac{2 \pi}{3}+\phi_{g r}\right) \\
& v_{a r}=V_{r} \sin \left(\omega_{r} t-\frac{2 \pi}{3}+\phi_{g r}\right),
\end{aligned}
$$

where $\phi_{g r}$ is the phase of voltage $v_{a r}$ and $V_{r}$ is the RMS rotor voltage.

The converter sets the rotor voltage according to the PWM modulation depth, $P_{m 2}$, delivered by the controller. The RMS rotor voltage magnitude $V_{r}$ can be expressed in terms of dc-link voltage as

$$
V_{r}=P_{m 2} \frac{\sqrt{3} V_{D C}}{2 \sqrt{2}} .
$$

In the $d q$-reference frame, this means that

$$
\begin{aligned}
& v_{d r}=P_{m d} \frac{\sqrt{3} V_{D C}}{2 \sqrt{2}}, \\
& v_{q r}=P_{m q} \frac{\sqrt{3} V_{D C}}{2 \sqrt{2}} .
\end{aligned}
$$

where $P_{m d}$ and $P_{m q}$ are the modulation depth's components. 


\section{Chapter 5}

\section{Control strategy}

In this work, two quantities are controlled: either active power and reactive power or active power and bus voltage. In this chapter, a control strategy will be described based on the third order model derived in section 3.1 .

\subsection{Controlling active power}

The rotor power is limited, compared to the stator power as shown in equation (3.24). Therefore, active power can be controlled by acting upon stator power which can be expressed as

$$
P_{s}=v_{d s} i_{d s}+v_{q s} i_{q s}
$$

The aim here is to control active power by means of rotor currents. In the above equation, the stator voltage is an input, obtained by simply measuring it, but the stator currents must be expressed in terms of rotor currents and stator voltage. To do so, equation (2.80) is solved for stator currents:

$$
\bar{\imath}_{d q s}=-\frac{X_{m} \bar{\imath}_{d q r}+\bar{\psi}_{d q s}}{X_{s}}
$$

Recalling the expression of stator fluxes in the third order model from equation (3.18):

$$
\bar{\imath}_{d q s}=-\frac{X_{m} \bar{\imath}_{d q r}-j \bar{v}_{d q s}}{X_{s}},
$$

and using this expression in the above expression of stator power, we get

$$
P_{s}=-\frac{X_{m}}{X_{s}}\left(v_{d s} i_{d r}+v_{q s} i_{q r}\right) \text {. }
$$

This means that there exists a strong coupling between the rotor currents and the stator active power and therefore between rotor currents and active power.

\subsection{Controlling reactive power or voltage}

It has been seen in chapter 4 that the converters assure that no reactive power is flowing between the rotor windings and the grid. This means that the reactive power is only produced or consumed by the stator. The rotor reactive power is

$$
Q_{s}=\operatorname{Im}\left(\bar{v}_{d q s} \bar{\imath}_{d q s}^{*}\right)=v_{q s} i_{d s}-v_{d s} i_{q s} .
$$


Using the expression of stator currents obtained in equation (5.3), we get

$$
Q_{s}=\frac{X_{m}}{X_{s}}\left(v_{d s} i_{q r}-v_{q s} i_{d r}\right)-\frac{1}{X_{s}}\left|\bar{v}_{s}\right|^{2} .
$$

This relation shows how tightly reactive power and stator voltage are coupled. Either of these two values can be controlled. In the following, control of reactive power will be discussed but it can be noted that voltage can be controlled instead. In chapter 8 , both reactive power and voltage control will be studied.

\subsection{Stator flux reference frame}

To simplify the relations between stator reactive and active powers, a new change of reference frame is generally applied [6]. The new reference frame, called here the $x y$-reference frame, has its $x$-axis aligned with the stator flux vector and its $y$-axis leading by $\frac{\pi}{2}$. If the angle of the stator flux phasor is $\theta_{\psi}$ in the $d q$-reference frame, the transformation corresponds to a simple rotation by $\theta_{\psi}$ as pictured in figure 5.1 .

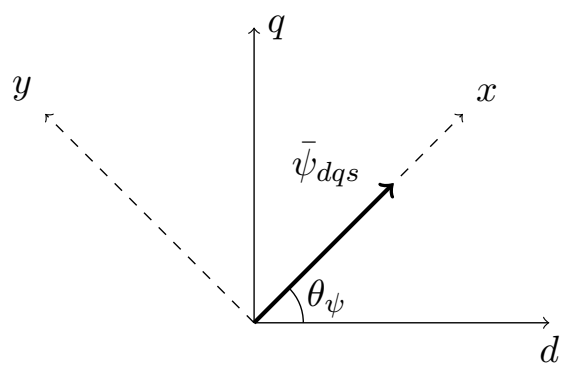

Figure 5.1: Stator-flux reference frame

The transformation between the $d q 0$-reference frame and the $x y$-reference frame is characterized by the following rotation matrix:

$$
\left[\begin{array}{c}
f_{x} \\
f_{y}
\end{array}\right]=\left[\begin{array}{cc}
\cos \left(\theta_{\psi}\right) & \sin \left(\theta_{\psi}\right) \\
-\sin \left(\theta_{\psi}\right) & \cos \left(\theta_{\psi}\right)
\end{array}\right]\left[\begin{array}{c}
f_{d} \\
f_{q}
\end{array}\right] .
$$

This means that in this new reference frame, the stator flux components are

$$
\bar{\psi}_{x y s}=\left[\begin{array}{c}
\psi_{s} \\
0
\end{array}\right],
$$

where $\psi_{s}$ is the module of the stator flux phasor.

The simple relation between stator voltages and stator fluxes in (3.18) holds in the new reference frame since it is defined by rotating the $d q$-reference frame. In this new reference frame, the stator voltage is:

$$
\bar{v}_{x y s}=\left[\begin{array}{c}
0 \\
v_{s}
\end{array}\right]
$$

where $v_{s}$ is the module of the stator voltage phasor. 
This transformation is power invariant. In the new reference frame, equations (5.4) and (5.6) become:

$$
\begin{aligned}
P_{s} & =-\frac{X_{m}}{X_{s}} v_{s} i_{y r}, \\
Q_{s} & =-\frac{X_{m}}{X_{s}} v_{s} i_{x r}-\frac{1}{X_{s}} v_{s}^{2},
\end{aligned}
$$

where $i_{x r}$ and $i_{y r}$ are the components of the rotor current phasor along the $x$ - and $y$-axes, respectively.

Therefore, in this new reference frame, active power is controlled by acting upon the $y$ component of rotor current and reactive power (or voltage) by acting upon the $x$-component of rotor current.

\subsection{Acting upon rotor currents}

The rotor currents have been used as control quantities. However, the rotor-side converter controls the rotor voltage. The dynamics of the rotor currents can be derived.

Using equations (3.18) and (15.2), the rotor fluxes can be expressed as

$$
\bar{\psi}_{d q r}=-\frac{X_{m}}{X_{s}}\left(j \bar{v}_{d q s}\right)-X^{\prime} \frac{X_{r}}{X_{s}} \bar{i}_{d q r} .
$$

The state variable $\bar{E}^{\prime}$ can then be expressed as a function of rotor currents by using its definition (3.4):

$$
\left[\begin{array}{c}
E_{d}^{\prime} \\
E_{q}^{\prime}
\end{array}\right]=\frac{1}{X_{s}}\left(\left(X_{s}-X^{\prime}\right)\left[\begin{array}{c}
v_{d s} \\
v_{q s}
\end{array}\right]-X^{\prime} X_{m}\left[\begin{array}{c}
-i_{q r} \\
i_{d r}
\end{array}\right]\right) .
$$

Substituting this expression into (3.20):

$$
\begin{aligned}
\frac{d}{d t}\left[\begin{array}{c}
i_{d r} \\
i_{q r}
\end{array}\right]= & \frac{1}{T_{0}}\left[\begin{array}{cc}
-\frac{X_{s}}{X^{\prime}} & s \omega_{s} T_{0} \\
-s \omega_{s} T_{0} & -\frac{X_{s}}{X^{\prime}}
\end{array}\right]\left[\begin{array}{c}
i_{d r} \\
i_{q r}
\end{array}\right] \\
& -\frac{X_{s}}{X^{\prime} X_{m}} \omega_{s}\left[\begin{array}{cccc}
-s \frac{X_{s}-X^{\prime}}{X_{s}} & 0 & \frac{X_{m}}{X_{r}} & 0 \\
0 & -s \frac{X_{s}-X^{\prime}}{X_{s}} & 0 & \frac{X_{m}}{X_{r}}
\end{array}\right]\left[\begin{array}{c}
v_{d s} \\
v_{q s} \\
v_{d r} \\
v_{q r}
\end{array}\right] .
\end{aligned}
$$

This equation can be rewritten:

$$
\frac{d}{d t} \bar{\imath}_{d q r}=\omega_{s} \frac{X_{s}}{X^{\prime}}\left(-\frac{1}{T_{0} \omega_{s}} \bar{\imath}_{d q r}-\frac{1}{X_{r}} \bar{v}_{d q r}+s\left(\frac{X_{s}-X^{\prime}}{X_{s} X_{m}} \bar{v}_{d q s}-\frac{X^{\prime}}{X^{s}}\left(j \bar{\imath}_{d q r}\right)\right)\right),
$$

and recalling the equation which links $\bar{E}^{\prime}$ and $\bar{v}_{d q s}$ and $\bar{\imath}_{d q r}$ from (5.13), we get

$$
\frac{d}{d t} \bar{\imath}_{d q r}=\omega_{s} \frac{X_{s} X_{m}}{X^{\prime}}\left(-\frac{X_{m}}{T_{0} \omega_{s}} \bar{\imath}_{d q r}-\frac{X_{m}}{X_{r}} \bar{v}_{d q r}+s \bar{E}^{\prime}\right),
$$

which allows to express the rotor voltages as, recalling the definition of $T_{0}$ in (3.11):

$$
\bar{v}_{d q r}=-R_{r} \bar{\imath}_{d q r}-\frac{X^{\prime} X_{r}}{\omega_{s} X_{s}} \frac{d \bar{\imath}_{d q r}}{d t}+s \frac{X_{r}}{X_{m}} \bar{E}^{\prime}
$$

This relation holds in the $x y$-reference frame introduced in section 5.3. Therefore, in the following, the $d$ and $q$ indices are replaced by $x$ and $y$. The term $s \frac{X_{r}}{X_{m}} \bar{E}^{\prime}$ can be interpreted as compensating term [12]. 


\subsection{Control blocks}

In the $x y$-reference frame (aligned with the stator flux), the controls of active and reactive powers are decoupled in the sense that the rotor current's $x$-component controls the reactive, while the $y$-component controls active power.

We use two PI controllers to deliver control signals which give the modulation depth components to be applied in the rotor-side converter. This strategy is pictured in figure 5.2 .

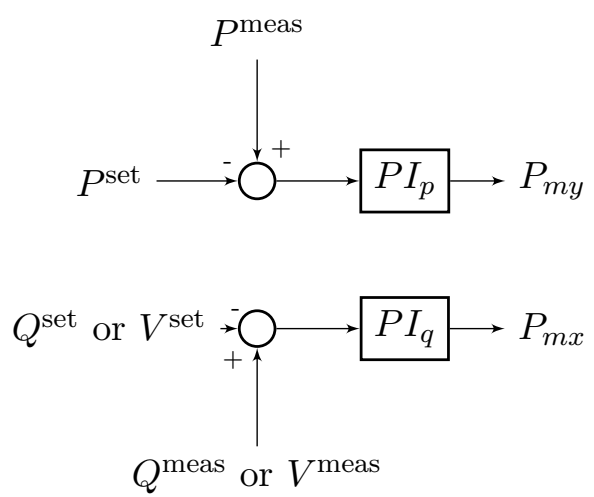

Figure 5.2: Control strategy

The quantities $P^{\text {set }}, Q^{\text {set }}$ and $V^{\text {set }}$ are the setpoint values of total active and reactive powers and of stator voltage, respectively. The quantities $P^{\text {meas }}, Q^{\text {meas }}$ and $V^{\text {meas }}$ are the actual total active and reactive power productions and the actual stator voltage, measured from the grid. This actually means controlling the voltages $v_{x r}$ and $v_{y r}$ using $P_{m d}$ and $P_{m q}$ in equation (4.9) and (4.10).

\subsection{Single machine equivalent signal}

A system with two sets of generators, $S_{1}$ and $S_{2}$, is considered. The set $S_{1}$ contains the critical generators and $S_{2}$ the non-critical generators, as defined in [5]. The following quantities are defined:

$$
\begin{array}{rlrl}
\delta_{C}= & \frac{\sum_{\mathrm{i} \in S_{1}} H_{i} \delta_{i}}{\sum_{\mathrm{i} \in S_{1}} H_{i}} & \omega_{C} & =\frac{\sum_{\mathrm{i} \in S_{1}} H_{i} \omega_{i}}{\sum_{\mathrm{i} \in S_{1}} H_{i}} \\
\delta_{N C}=\frac{\sum_{\mathrm{i} \in S_{2}} H_{i} \delta_{i}}{\sum_{\mathrm{i} \in S_{2}} H_{i}} & \omega_{N C}=\frac{\sum_{\mathrm{i} \in S_{2}}}{\sum_{\mathrm{i} \in S_{2}} H_{i}}
\end{array}
$$

The subscripts $C$ and $N C$ stand for critical and non-critical, respectively. The symbols $\delta_{i}$, $\omega_{i}$ and $H_{i}$ denote the rotor angle, the angular speed and the inertia of generator $i$. From these quantities, the two following values are defined:

$$
\begin{aligned}
\delta_{\text {sime }} & =\delta_{C}-\delta_{N C} \\
\omega_{\text {sime }} & =\omega_{C}-\omega_{N C}
\end{aligned}
$$


The single machine equivalent (SIME) signal can be built based on these two quantities:

$$
y_{\text {sime }}=\sin \left(\delta_{\text {sime }}\right) \omega_{\text {sime }}
$$

This signal can be amplified and is summed up with the signals from the PI-controllers as shown in figure 5.3. The amplification gains $K_{S p}$ and $K_{S q}$ (or $K_{S v}$ if voltage is controlled instead of reactive power) can be different.

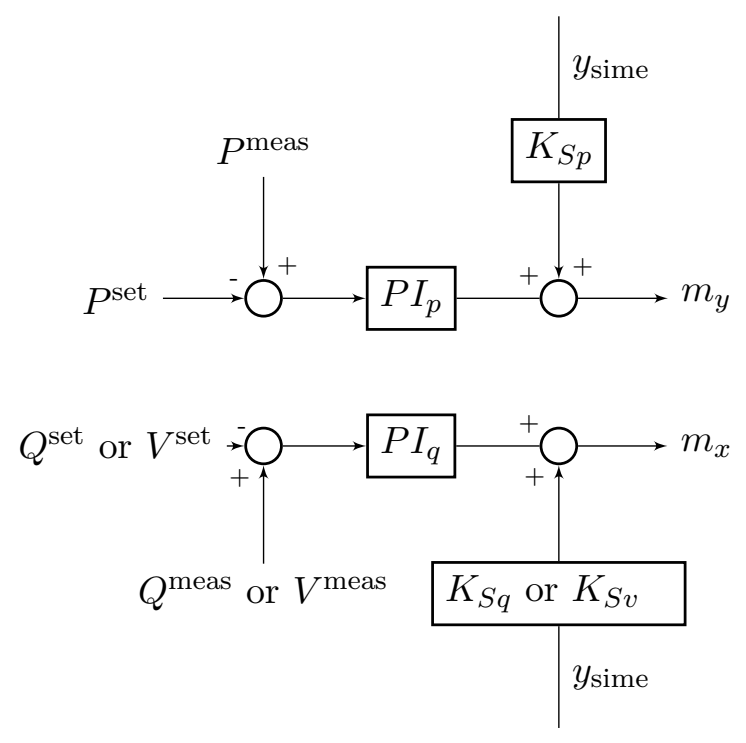

Figure 5.3: Control strategy with additional signal

The signal may add damping in the system if it is properly tuned.

\subsection{Switching between reference frames}

All along this work, different reference frames have been used. On the one hand, the synchronous reference frame (so-called $d q 0$-reference frame) is used for deriving the machine model. On the other hand, the stator-flux reference frame (so-called $x y$-reference frame) is used for controlling the machine. This means that the control signals, calculated in the $x y$-reference frame, must be transformed into the synchronous reference frame. This transformation is fully described by equation (5.7). In order to rotate the components back to the synchronous reference frame we can just consider the above mentioned equation with opposite angle:

$$
\left[\begin{array}{l}
f_{d} \\
f_{q}
\end{array}\right]=\left[\begin{array}{cc}
\cos \left(\theta_{\psi}\right) & -\sin \left(\theta_{\psi}\right) \\
\sin \left(\theta_{\psi}\right) & \cos \left(\theta_{\psi}\right)
\end{array}\right]\left[\begin{array}{l}
f_{x} \\
f_{y}
\end{array}\right] .
$$

Power Factory, the program used for the simulations, works with a reference frame aligned with the rotor angle of the reference machine (termed here as slack reference frame, see figure 5.4).

In the figure, $S_{x}$ and $S_{y}$ are the two axes of this slack reference frame rotating at $\omega_{\text {slack}}$, speed of the reference machine. The stator flux angle, $\theta_{\psi}^{S}$, is available in Power Factory only 


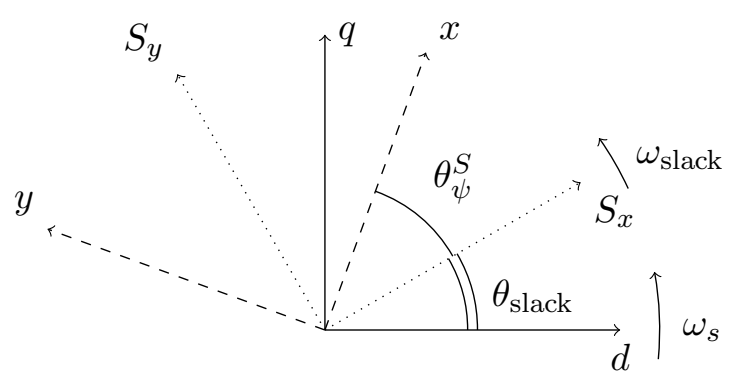

Figure 5.4: Different reference frames

in the slack reference frame. The angle of the reference machine in the synchronous reference frame can then be calculated as:

$$
\theta_{\text {slack }}(t)=\int_{0}^{t}\left(\omega_{\text {slack }}-\omega_{s}\right) d t .
$$

Because the stator flux angle is only available in the slack reference frame, the quantities are not rotated directly from the $x y$-reference frame to the synchronous reference frame but first from the $x y$-reference frame to the slack reference frame and then from the slack reference frame to the synchronous reference frame. This can be seen as a single rotation by atal angle of $\theta_{\psi}^{S}+\theta_{\text {slack }}$ and can be expressed as:

$$
\left[\begin{array}{c}
P_{m d} \\
P_{m q}
\end{array}\right]=\left[\begin{array}{cc}
\cos \left(\theta_{\psi}^{S}+\theta_{\text {slack }}\right) & -\sin \left(\theta_{\psi}^{S}+\theta_{\text {slack }}\right) \\
\sin \left(\theta_{\psi}^{S}+\theta_{\text {slack }}\right) & \cos \left(\theta_{\psi}^{S}+\theta_{\text {slack }}\right)
\end{array}\right]\left[\begin{array}{c}
P_{m x} \\
P_{m y}
\end{array}\right]
$$

With this transformation, the modulation depths can therefore be rotated in the synchronous reference frame where the equations describing the machine are valid.

However, the modulation depths $P_{m x}$ and $P_{m y}$ are not the only quantities that need to be rotated. When looking at the electrical dynamics in equation (3.20), stator voltage is also considered as input, which means that it is given by default in the slack reference frame in Power Factory. The voltage must therefore also be rotated from the slack reference frame to the synchronous reference frame. 


\section{Chapter 6}

\section{DFIG model}

In this chapter, we will develop our own DFIG model, based on the equations presented previously in this thesis.

\subsection{Assumptions}

First of all, the DC-link voltage will be considered constant. Moreover we neglect the stator resistance as we have done before in section 3.1.3. The role of the grid-side converter is to assure operation of the rotor side at unity factor in our case (see section 4), and only active power is transmitted to the grid.

\subsection{Equivalent circuit}

The model developed in this work is built upon a voltage source, which is controlled such that it produces the same amount of power than an actual DFIG. The DFIG and its equivalent model are represented in figure 6.1,

It may be emphasized that this equivalent circuit is completely different from the one depicted in figure 3.2. The latter is an equivalent model of the stator side only. The model presented here is an equivalent model of the whole generator. It represents both the rotor and the stator. Specifically, the equivalent voltage $\bar{E}^{\prime}$ and the impedance $X^{\prime}$ on the one hand, and $\bar{E}_{e q}$ and $X_{e q}$ on the other hand are two distinct couples of quantities. The reactance $X_{e q}$ can be arbitrarily chosen.

One of the greatest advantages with this approach is that most power system simulation software provides built-in controlled voltage source models. This means that we can even use software which does not allow us to create our own components to carry out the comparison. It is especially important in the scope of this thesis because the simulation software we use, DigSilent PowerFactory, is one of this kind. It allows the user to create control schemes for the existing components but the creation of completely new components is not allowed. We will therefore use the built-in voltage source and control it to emulate the DFIG's behavior. We use the strategy depicted in figure 6.2. In this figure, $E_{e q}$ and $\delta$ represent the controlled source's voltage magnitude and angle, respectively.

We start from the control signals from the controllers (the PWM modulation depth's components) which act upon the rotor voltage. From these, we use the DFIG equations to calculate the theoretical active and reactive power transmitted to the grid. The total powers transmitted 


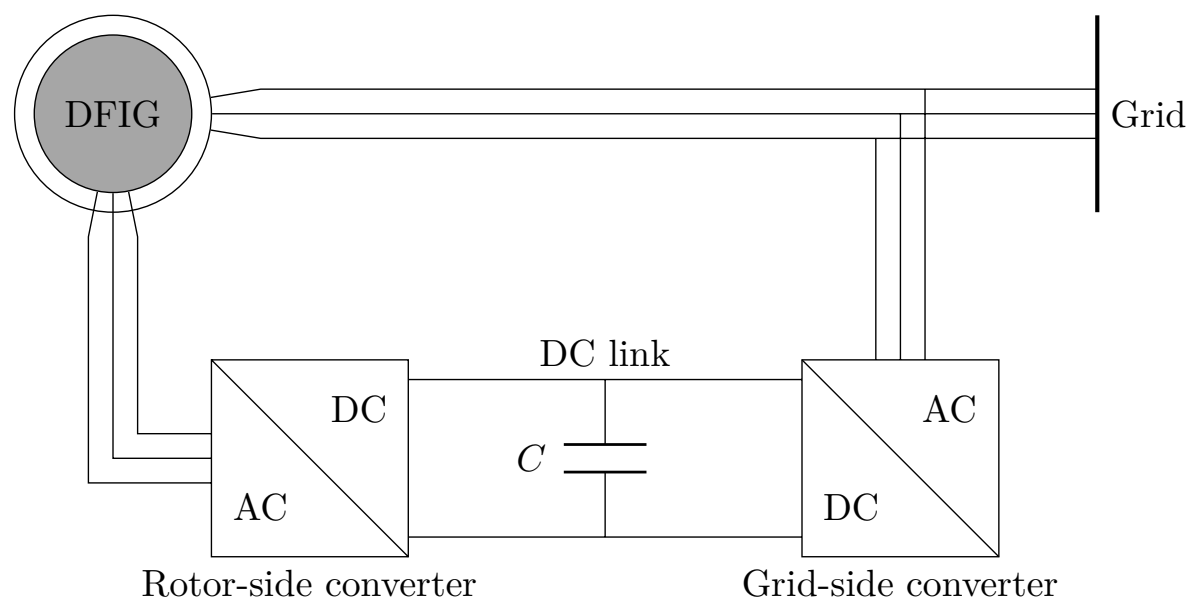

(a) DFIG detailed model

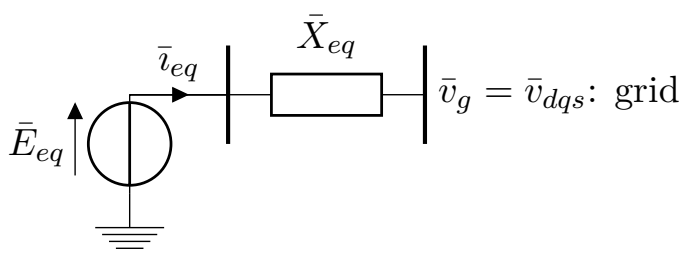

(b) Equivalent circuit

Figure 6.1: Equivalent circuit of the first order model

to the grid are

$$
\begin{aligned}
P_{g} & =P_{s}+P_{r}, \\
Q_{g} & =Q_{s} .
\end{aligned}
$$

Once these powers are obtained, we calculate the voltage which must be set by the equivalent controlled voltage source to actually feed the grid with these powers. The actual powers transmitted to the grid are then measured, compared with the setpoints and used by the control scheme to compute the control signals $P_{m x}$ and $P_{m y}$. All steps are described in detail in the following sections.

\subsection{First- and third-order models}

The model developed here can actually be used to create models of any order, and in particular of first- and third-orders. The only difference is whether the electrical dynamics, that is the dynamics of $\bar{E}^{\prime}$, are taken into account. The first-order model is presented but will not be used in the simulations in this work. 


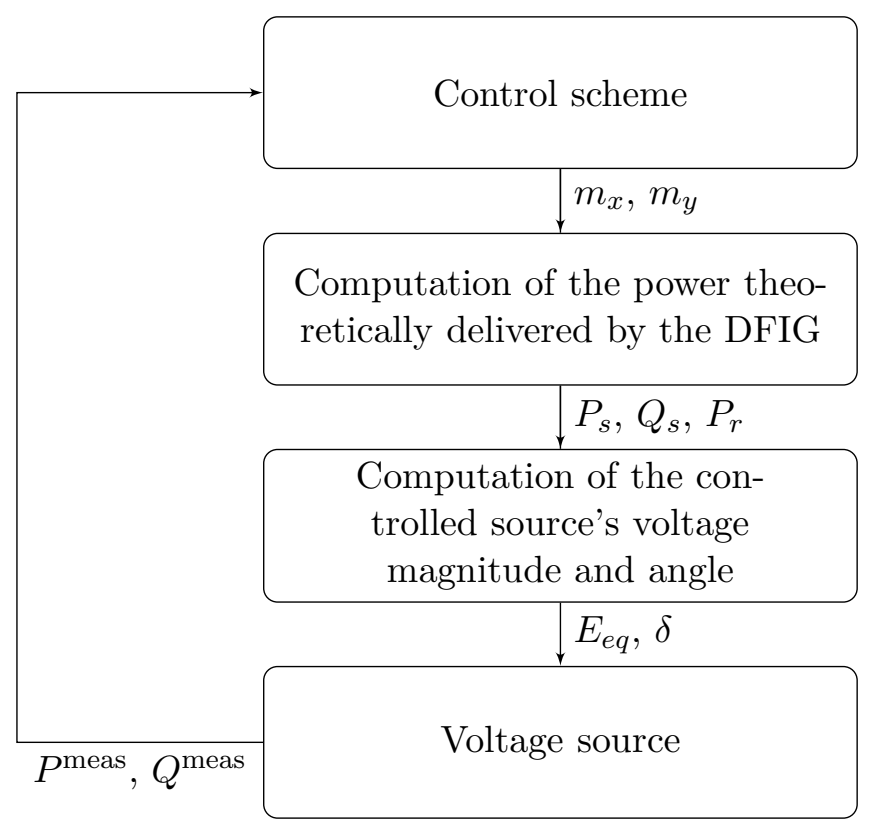

Figure 6.2: Strategy to compute $E_{e q}$ and $\delta$

\subsubsection{First-order model}

If the first-order model is considered, from (3.20) and remembering that we have neglected $R_{s}$, the following relations hold:

$$
\begin{aligned}
& 0=-\frac{X_{s}}{X^{\prime}} E_{d}^{\prime}+s \omega_{s} T_{0} E_{q}^{\prime}+\frac{X_{s}-X^{\prime}}{X^{\prime}} v_{d s}-\omega_{s} \frac{X_{m}}{X_{r}} T_{0} v_{q r}, \\
& 0=-\frac{X_{s}}{X^{\prime}} E_{q}^{\prime}-s \omega_{s} T_{0} E_{d}^{\prime}+\frac{X_{s}-X^{\prime}}{X^{\prime}} v_{q s}+\omega_{s} \frac{X_{m}}{X_{r}} T_{0} v_{d r} .
\end{aligned}
$$

The system can be put in the more handy matrix form:

$$
\begin{aligned}
{\left[\begin{array}{cc}
-\frac{X_{s}}{X^{\prime}} & s \omega_{s} T_{0} \\
-s \omega_{s} T_{0} & -\frac{X_{s}}{X^{\prime}}
\end{array}\right]\left[\begin{array}{c}
E_{d}^{\prime} \\
E_{q}^{\prime}
\end{array}\right] } & =\left[\begin{array}{c}
-\frac{X_{s}-X^{\prime}}{X^{\prime}} v_{d s}+\omega_{s} \frac{X_{m}}{X_{r}} T_{0} v_{q r} \\
-\frac{X_{s}-X^{\prime}}{X^{\prime}} v_{q s}-\omega_{s} \frac{X_{m}}{X_{r}} T_{0} v_{d r}
\end{array}\right] \\
\boldsymbol{A}\left[\begin{array}{c}
E_{d}^{\prime} \\
E_{q}^{\prime}
\end{array}\right] & =\boldsymbol{B} .
\end{aligned}
$$

Inverting the system to get $\bar{E}^{\prime}$ :

$$
\begin{aligned}
\boldsymbol{A}^{-1} & =\frac{1}{\operatorname{det}(\boldsymbol{A})} \operatorname{adj}(\boldsymbol{A}) \\
& =\frac{1}{\frac{X_{s}^{2}}{X^{\prime 2}}+\left(s \omega_{s} T_{0}\right)^{2}}\left[\begin{array}{cc}
-\frac{X_{s}}{X^{\prime}} & -s \omega_{s} T_{0} \\
s \omega_{s} T_{0} & -\frac{X_{s}}{X^{\prime}}
\end{array}\right], \\
{\left[\begin{array}{c}
E_{d}^{\prime} \\
E_{q}^{\prime}
\end{array}\right] } & =\boldsymbol{A}^{-1} \boldsymbol{B} \\
& =\frac{1}{\frac{X_{s}^{2}}{X^{\prime 2}}+\left(s \omega_{s} T_{0}\right)^{2}}\left[\begin{array}{cc}
-\frac{X_{s}}{X^{\prime}} & -s \omega_{s} T_{0} \\
s \omega_{s} T_{0} & -\frac{X_{s}}{X^{\prime}}
\end{array}\right]\left[\begin{array}{l}
-\frac{X_{s}-X^{\prime}}{X^{\prime}} v_{d s}+\omega_{s} \frac{X_{m}}{X_{r}} T_{0} v_{q r} \\
-\frac{X_{s}-X^{\prime}}{X^{\prime}} v_{q s}-\omega_{s} \frac{X_{m}}{X_{r}} T_{0} v_{d r}
\end{array}\right] .
\end{aligned}
$$


The quantity $\bar{E}^{\prime}$ can therefore be calculated using $\bar{v}_{s}$, as a measured input, and $\bar{v}_{r}$, calculated from the control signals as seen in equations (4.9) and (4.10).

\subsubsection{Third-order model}

If the third-order model is now considered, $\bar{E}^{\prime}$ is calculated by solving the two differential equations describing the electrical dynamics in (3.20), which are recalled below:

$$
\begin{aligned}
& \frac{d E_{d}^{\prime}}{d t}=\frac{1}{T_{0}}\left(-\frac{X_{s}}{X^{\prime}} E_{d}^{\prime}+s \omega_{s} T_{0} E_{q}^{\prime}+\frac{X_{s}-X^{\prime}}{X^{\prime}} v_{d s}-T_{0} \omega_{s} \frac{X_{m}}{X_{r}} v_{q r}\right), \\
& \frac{d E_{q}^{\prime}}{d t}=\frac{1}{T_{0}}\left(-\frac{X_{s}}{X^{\prime}} E_{q}^{\prime}-s \omega_{s} T_{0} E_{d}^{\prime}+\frac{X_{s}-X^{\prime}}{X^{\prime}} v_{q s}+T_{0} \omega_{s} \frac{X_{m}}{X_{r}} v_{d r}\right) .
\end{aligned}
$$

No literal expressions can be derived for $E_{d}^{\prime}$ and $E_{q}^{\prime}$ but the differential equations can be solved numerically by a computation software (PowerFactory offers such a solver).

\subsection{Theoretical power delivered by the first order model}

In this section, we want to calculate the powers which an actual DFIG would produce if it was fed with given control signals $P_{m x}$ and $P_{m y}$.

\subsubsection{Calculation of the stator active power}

The model and the equations which we have derived are all defined in the $d q$-reference frame rotating at synchronous speed. This means that the first step is to rotate the modulation depth's components $\left(P_{m x}\right.$ and $\left.P_{m y}\right)$ from the stator-flux reference frame back to the synchronous reference frame, using relation (5.7). Then, starting from the obtained $P_{m d}$ and $P_{m q}$ :

$$
\begin{aligned}
& v_{d r}=\frac{\sqrt{3}}{2 \sqrt{2}} P_{m d} V_{d c}, \\
& v_{q r}=\frac{\sqrt{3}}{2 \sqrt{2}} P_{m q} V_{d c},
\end{aligned}
$$

and the stator active power is expressed by

$$
P_{s}=i_{d s} v_{d s}+i_{q s} v_{q s} .
$$

In the equations above, the DC-link voltage is assumed constant and known. We now consider the stator equivalent circuit in figure 3.2. We insist here one more time that this is the stator equivalent circuit and not the DFIG equivalent circuit of figure 6.1 which is considered. The stator current can be expressed as

$$
\bar{\imath}_{s}=\left[\begin{array}{c}
i_{d s} \\
i_{q s}
\end{array}\right]=\frac{\bar{E}^{\prime}-\bar{v}_{s}}{j X^{\prime}}=\frac{1}{X}\left(\left[\begin{array}{c}
-v_{q s} \\
v_{d s}
\end{array}\right]-\left[\begin{array}{c}
-E_{q}^{\prime} \\
E_{d}^{\prime}
\end{array}\right]\right) .
$$

The expression of $\bar{E}^{\prime}$ is then used in equation (6.16). We get thereby the stator active power which has to be produced by the controlled voltage source.

\subsubsection{Calculation of the stator reactive power}

The stator reactive power is expressed as

$$
Q_{s}=v_{q s} i_{d s}-v_{d s} i_{q s}
$$

with $\bar{\imath}_{d q s}$ expressed by equation (6.16). 


\subsubsection{Calculation of the rotor active power}

The rotor active power is expressed as

$$
P_{r}=v_{r d} i_{d r}+v_{r q} i_{q r}
$$

We want to express it in terms of known values (i.e $\bar{E}^{\prime}$ and $\bar{v}_{s}$ in this case). From (5.3), we have

$$
\bar{\imath}_{s}=\frac{j \bar{v}_{s}-X_{m} \bar{\imath}_{r}}{X_{s}}
$$

which means that

$$
\bar{\imath}_{r}=\frac{1}{X_{m}}\left(j \bar{v}_{s}-X_{s} \bar{v}_{s}\right) .
$$

Recalling the expression of the stator current from (6.16), the rotor currents can be expressed as

$$
\bar{\imath}_{r}=\left[\begin{array}{c}
i_{d r} \\
i_{q r}
\end{array}\right]=\frac{1}{X_{m} X^{\prime}}\left(\left(X^{\prime}-X_{s}\right) j \bar{v}_{s}+j X_{s} \bar{E}^{\prime}\right),
$$

which means that

$$
\left[\begin{array}{c}
i_{d r} \\
i_{q r}
\end{array}\right]=\frac{1}{X_{m} X^{\prime}}\left(\left(X^{\prime}-X_{s}\right)\left[\begin{array}{c}
-v_{q s} \\
v_{d s}
\end{array}\right]+X_{s}\left[\begin{array}{c}
-E_{q}^{\prime} \\
E_{d}^{\prime}
\end{array}\right]\right)
$$

\subsection{Controlled voltage source}

In the previous section, the theoretical powers supposed to be delivered by the model in response to the control signals $P_{m d}$ and $P_{m q}$ have been calculated. Therefore, $P_{s}, Q_{s}$ and $P_{r}$ are known. With the notations defined in figure 6.1, the powers flowing into the grid are

$$
\begin{aligned}
P_{g} & =\operatorname{Re}\left(\bar{v}_{d q s} \bar{\imath}_{e q}^{*}\right), \\
Q_{g} & =\operatorname{Im}\left(\bar{v}_{d q s} \bar{\imath}_{e q}^{*}\right),
\end{aligned}
$$

and

$$
\bar{\imath}_{e q}=\frac{\bar{E}_{e q}-\bar{v}_{d q s}}{j X_{e q}} .
$$

We define the phasor quantities $\bar{E}_{e q}$ and $\bar{v}_{s}$ as

$$
\begin{aligned}
\bar{E}_{e q} & =E_{e q} e^{j \delta}, \\
\bar{v}_{d q s} & =V_{s} e^{j \theta} .
\end{aligned}
$$

This means that

$$
\bar{v}_{d q s} \bar{\imath}_{e q}^{*}=-\frac{V_{s} E_{e q}}{X_{e q}} \sin (\theta-\delta)+j\left(\frac{V_{s} E_{e q}}{X_{e q}} \cos (\theta-\delta)-\frac{V_{s}^{2}}{X_{e q}}\right),
$$

so that

$$
\bar{v}_{d q s} \bar{\imath}_{e q}^{*}=-\frac{V_{s} E_{e q}}{X_{e q}} \sin (\theta-\delta)+j\left(\frac{V_{s} E_{e q}}{X_{e q}} \cos (\theta-\delta)-\frac{V_{s}^{2}}{X_{e q}}\right)
$$


and finally:

$$
\begin{gathered}
P_{g}=P_{s}+P_{r}=-\frac{V_{s} E_{e q}}{X_{e q}} \sin (\theta-\delta), \\
Q_{g}=Q_{s}=\frac{V_{s} E_{e q}}{X_{e q}} \cos (\theta-\delta)-\frac{V_{s}^{2}}{X_{e q}} .
\end{gathered}
$$

This could be solved by a numerical computing software to get $E_{e q}$ and $\delta$. However the simulation software we use does not allow us to solve directly these equations. We will solve the system analytically to obtain direct expressions of $E_{e q}$ and $\delta$.

Starting from (6.30), we want to calculate $E_{e q}$ and $\delta$. Let us first extract an expression of $E_{e q}$. Taking the square of both the active and the reactive power and summing them leads to

$$
\begin{aligned}
X_{e q}\left(P_{g}^{2}+Q_{g}^{2}\right) & =\left(V E_{e q}\right)^{2} \sin ^{2}(\theta-\delta) \\
& +\left(V E_{e q}\right)^{2} \cos ^{2}(\theta-\delta)+V^{4}-2 V^{3} E_{e q} \cos (\theta-\delta) \\
& =\left(V E_{e q}\right)^{2}+V^{4}-2 V^{3} E_{e q} \cos (\theta-\delta) .
\end{aligned}
$$

The cosine component can be expressed from the reactive power as:

$$
2 V^{3} E_{e q} \cos (\theta-\delta)=2 X_{e q} V^{2}\left(Q_{g}+\frac{V^{2}}{X_{e q}}\right)
$$

and used in the sum of the squared powers:

$$
X_{e q}\left(P_{g}^{2}+Q_{g}^{2}\right)=\left(V E_{e q}\right)^{2}+V^{4}-2 X_{e q} V^{2}\left(Q_{g}+\frac{V^{2}}{X_{e q}}\right) .
$$

This can be solved for $E_{e q}^{2}$ :

$$
E_{e q}^{2}=\frac{1}{V^{2}}\left(X^{2}\left(P_{g}^{2}+Q_{g}^{2}\right)+2 X_{e q} V^{2}\left(Q_{g}+\frac{V^{2}}{X_{e q}}\right)-V^{4}\right) .
$$

We get finally

$$
E_{e q}=\sqrt{E_{e q}^{2}}
$$

The angle $\delta$ is now to be calculated. We start from

$$
\begin{aligned}
& \sin (\theta-\delta)=-\frac{X_{e q} P_{g}}{V E_{e q}} \\
& \cos (\theta-\delta)=\frac{X_{e q}}{V E_{e q}}\left(Q_{g}+\frac{V^{2}}{X_{e q}}\right) .
\end{aligned}
$$

The quadrant of the complex plane in which $\bar{E}_{e q}$ lies needs to be determined carefully. It is known that if the cosine is positive then the angle can be obtained from the sine directly by the inverse function. Otherwise, the actual angle is actually $\pi$ added to the angle obtained by the inverse function. Precisely:

$$
\delta= \begin{cases}\theta-\arcsin \left(-\frac{X_{e q} P_{g}}{V E_{e q}}\right) & \text { if } \cos (\theta-\delta)>0, \\ \theta-\left(\pi-\arcsin \left(-\frac{X_{e q} P_{g}}{V E_{e q}}\right)\right) & \text { if } \cos (\theta-\delta)<0\end{cases}
$$


The controlled voltage and angle are therefore expressed as:

$$
\begin{aligned}
E_{e q}^{2} & =\frac{1}{V_{s}^{2}}\left(X^{2}\left(P_{g}^{2}+Q_{g}^{2}\right)+2 X_{e q} V_{s}^{2}\left(Q_{g}+\frac{V_{s}^{2}}{X_{e q}}\right)-V_{s}^{4}\right), \\
\delta & = \begin{cases}\theta-\arcsin \left(-\frac{X_{e q} P_{g}}{V_{s} E_{e q}}\right) & \text { if } \cos (\theta-\delta)>0, \\
\theta-\left(\pi-\arcsin \left(-\frac{X_{e q} P_{g}}{V_{s} E_{e q}}\right)\right) & \text { if } \cos (\theta-\delta)<0\end{cases}
\end{aligned}
$$

\subsection{Load flow calculations}

The controlled voltage source defined inside Power Factory is depicted in figure 6.3. The source and its associated reactance can be seen. The transformer makes the connection to the grid.

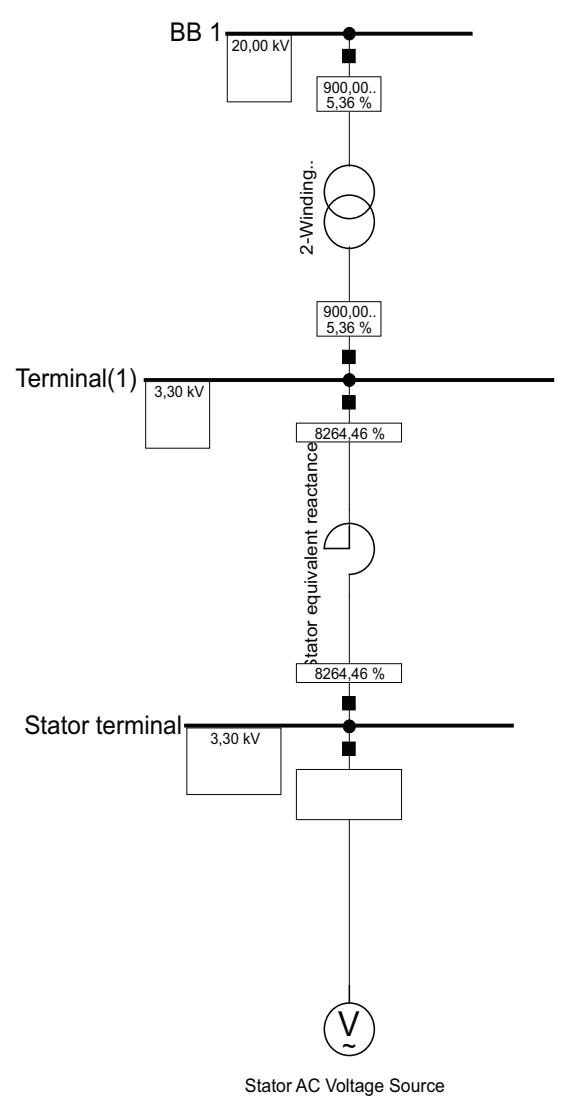

Figure 6.3: User-created model in Power Factory

Power Factory offers external load flow controllers for voltage sources. These components control voltage sources to set the value of either reactive power, active power, voltage angle or voltage magnitude at one specific bus. The latter should obviously be close to the voltage source itself for the load flow to converge. In this work, the voltage source is set as a PV bus for load flow calculations. 


\subsection{Initialization}

The equations derived in chapter 3 describe the dynamic behavior of the model. However, before that, the model must be initialized from the load flow values.

The set of equations which is solved during the initialization is:

$$
\begin{aligned}
0 & =-E_{d}^{\prime}+s \omega_{s} T_{0} E_{q}^{\prime}+\left(X_{s}-X_{p}\right) i_{q s}-T_{0} \omega_{s} \frac{X_{m}}{X_{r}} v_{d r} \\
0 & =-E_{q}^{\prime}-s \omega_{s} T_{0} E_{q}^{\prime}-\left(X_{s}-X_{p}\right) i_{q s}+T_{0} \omega_{s} \frac{X_{m}}{X_{r}} v_{d r} \\
s v_{s q} & =-X_{s} i_{q s}-X_{m} i_{d r} \\
v_{s d} & =X_{s} i_{q s}+X_{m} i_{q r} \\
\frac{X_{r}}{X_{m}} E_{q}^{\prime} & =-X_{m} i_{d s}-X_{r} i_{d r} \\
\frac{X_{r}}{X_{m}} E_{d}^{\prime} & =X_{m} i_{q s}+X_{r} i_{q r} \\
P_{g} & =v_{d s} i_{d s}+v_{q s} i_{q s}+v_{d r} i_{d r}+v_{q r} i_{q r} \\
Q_{g} & =v_{q s} i_{d s}-v_{d s} i_{q s} .
\end{aligned}
$$

Equations (6.42) and (6.43) represent the initialization of electrical dynamics. Equations (6.44) and (6.45) relate the stator voltage to the currents thanks to the stator flux equation and the relation between stator flux and stator voltage in (3.18). Equations (6.46) (6.47) are actually the rotor flux equations where $\bar{E}^{\prime}$ has replaced $\bar{\psi}_{r}$ using (3.4). The active and reactive powers transmitted to the grid are accounted for in the last two equations.

Table 6.1 presents the unknown quantities and the values which are known from the load flow.

Table 6.1: Unknowns and load flow values

\begin{tabular}{cc}
\hline \multicolumn{2}{c}{ Unknown quantities } \\
\hline Fictitious voltage $\bar{E}^{\prime}$ & $E_{d}^{\prime}, E_{d}^{\prime}$ \\
Rotor voltage & $v_{d r}, v_{q r}$ \\
Rotor current & $i_{d r}, i_{d r}$ \\
Rotor current & $i_{d s}, i_{q s}$ \\
\hline Known values \\
\hline Stator voltage & $v_{d s}, v_{q s}$ \\
Active power fed to the grid & $P_{g}$ \\
Reactive power fed to the grid & $Q_{g}$ \\
\hline
\end{tabular}

It may be noted that the load flow values of the powers transmitted to the grid $\left(P_{g}\right.$ and $\left.Q_{g}\right)$ are also used as the controller's setpoints for active and reactive powers $\left(P^{\text {set }}\right.$ and $Q^{\text {set }}$ in figure 5.2). This means that the control strategy strives to make the machine produce these powers at any time.

As mentioned before, the computer software we use, PowerFactory, does not solve systems of equations. The initial conditions are therefore solved in another computing software and the obtained numerical values are then used as initial values in PowerFactory. This adds complexity to this model. 


\subsection{Summary}

In this section, we want to summarize the strategy we have used to make our model based on a controllable voltage source.

We start from the control strategy depicted in figure 5.3, with or without the additional signal added after the PI controllers. This may be called step 1. From the signals given by the control scheme, we calculate the powers theoretically fed to the grid by an actual DFIG, using the equations which describe it. This is illustrated in figure 6.4 and may be referred to as step 2. Once we know what powers the controlled voltage source is supposed to supply to the grid, its voltage magnitude and angle can be calculated from equations (6.40) and (6.41). This so-called step 3 was described by figure 6.1. The actual produced powers are measured at the grid interface and fed back to the control system which then computes the control signals $P_{m x}$ and $P_{m y}$ (back to step 1). This model is termed Model $C$ in this thesis.

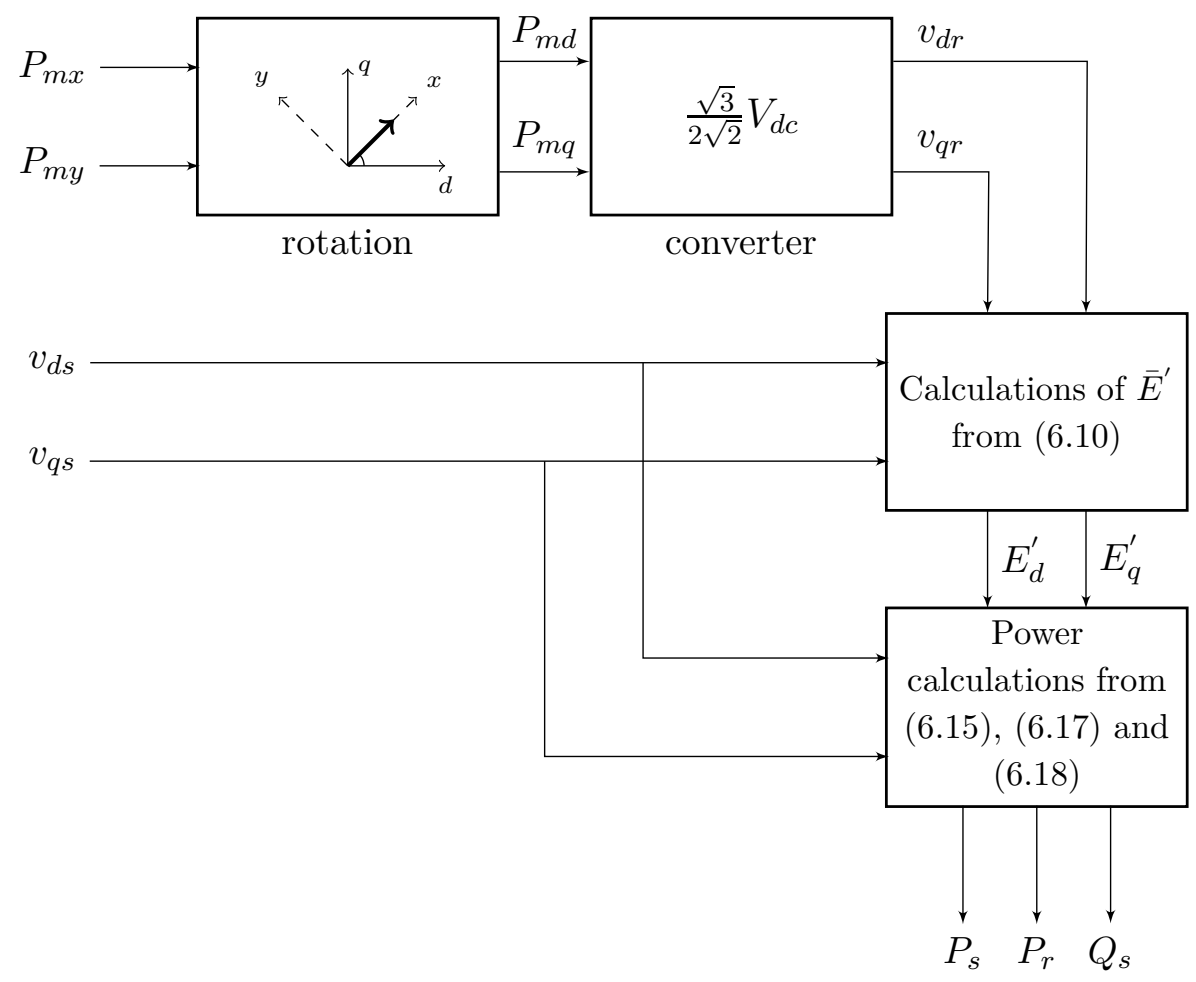

Figure 6.4: Theoretical power calculation process 



\section{Chapter 7}

\section{Built-in models}

Two built-in DFIG components exist in PowerFactory, one coming as a built-in example in version 13 and one as a built-in component. The first is depicted in figure 7.1 and will be referred to as Model $A$ in this thesis.

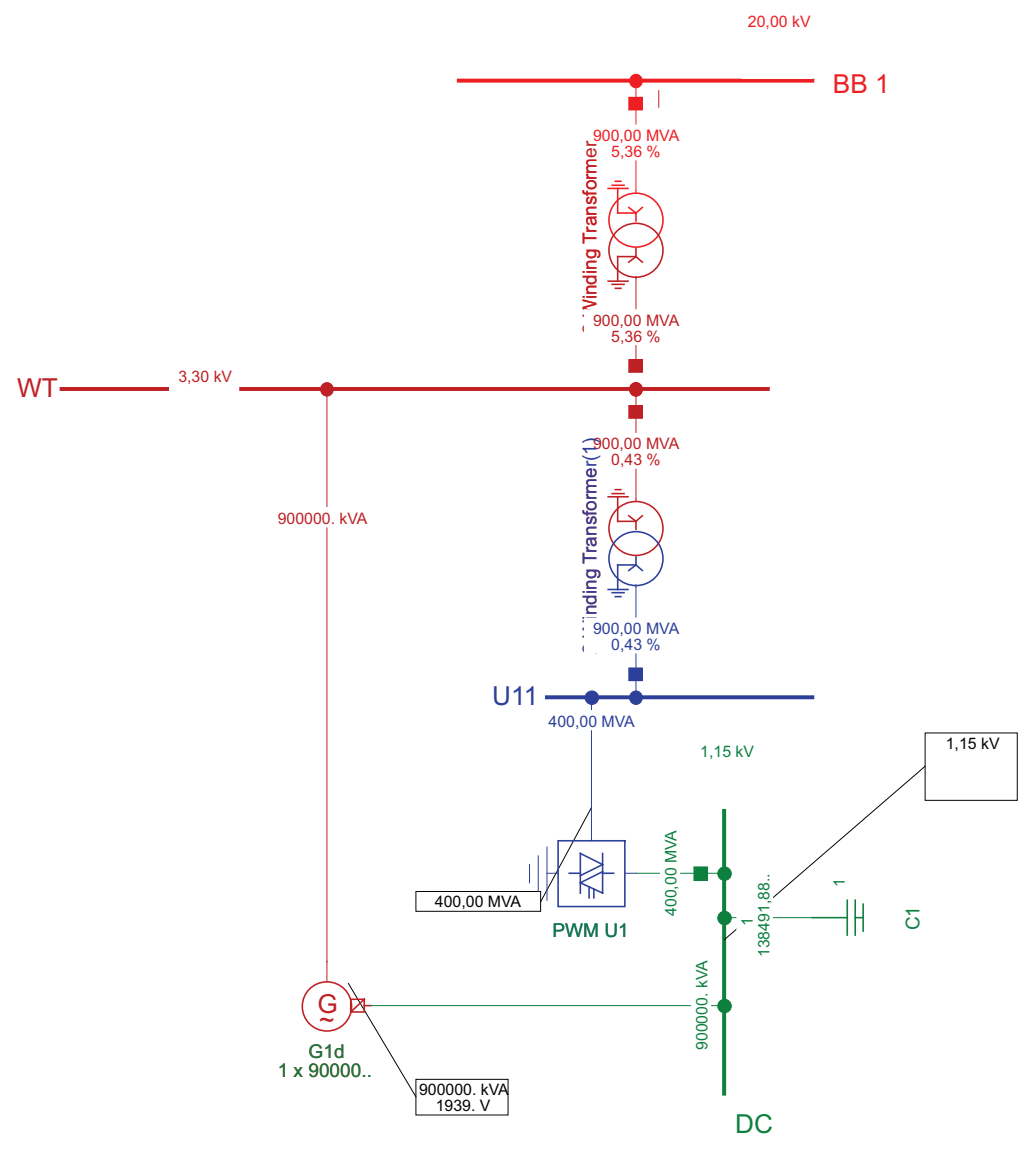

Figure 7.1: Built-in model with DC link in Power Factory 
As seen from the picture, this built-in model goes into more detail than our model. The DC-link voltage is controlled to be constant, whereas it is assumed to be constant in our model. Similarly, the rotor side's power factor is controlled to be one, whereas it is set to one in our model. The controller is however not instantaneous and small amounts of reactive power are either produced or consumed.

The built-in component is represented in figure 7.2 and will be termed Model B in this thesis.

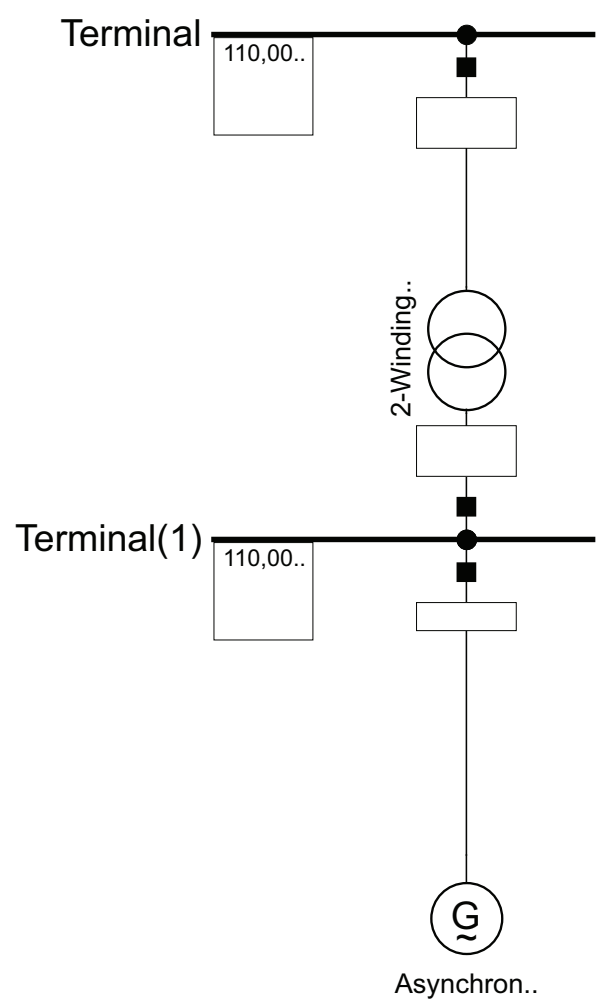

Figure 7.2: Built-in model without DC link in Power Factory

As in Model A, the rotor-side is controlled to run at unity power factor. However, in this model, the DC-link is hidden and the user does not have any control over the grid-side converter.

Other interesting differences exist, specifically concerning the load flow calculations. With Model $A$, it is only possible to define the reactive and active powers produced by the stator. There is no possibility to define it as a PV bus or even to define how much total power should be produced (by total power, it is here meant both stator and rotor powers). This puts some strong limitations on the load flow behavior of this model, since we are generally interested in total power and not only stator power.

With Model B however, the user is allowed to define the generator as a PV or PQ bus. It is even possible for the user to define the power flow at another bus. Thus, if we consider figure 7.2 once again, the power flowing after the transformer and to the grid can be defined. This model is therefore much more interesting than the other one. Of course, if one is interested in 
the properties of the DC link, it is then more convenient to work with the first model.

Finally, Power Factory allows the user to define a control strategy. The built-in components are controllable via input signals. Model $A$ is controlled by the modulation depths of the rotorside controller in a reference frame rotating with the rotor angle. With Model B, the user directly defines the two components of the rotor voltage (defined in the rotor-angle oriented reference frame). 



\section{Chapter 8}

\section{Simulations}

The first part of this work has been the opportunity to go through the classical representations of a DFIG and to develop a new model based upon a controllable voltage source. Power Factory's built-in models have been presented. We use simulations to compare these three models and to study the effect of the additional signal presented in section 5.6 ,

\subsection{Simulation setup}

\subsubsection{Grid}

We consider the two-area system depicted in figure 8.1 to carry out a comparison between the different models. We are interested in the differences in the models' behavior.

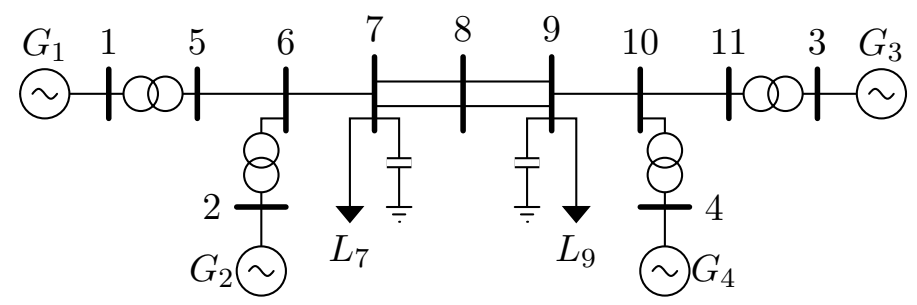

Figure 8.1: Two-area system

The system is composed of two areas, each containing two generators. Generators G2, G3 and G4 are synchronous generators. Generator G1 is a DFIG generator, either one of the built-in models or our model.

The synchronous generators are equipped with a governor depicted in figure 8.2 and whose values are presented in appendix $\mathrm{A}$ in table $\mathrm{A.1}$.

\subsubsection{Values used in the simulations}

Except for the values used for the DFIG, all values can be found in [9]. The values for the DFIG can be found in the appendix $\mathrm{A}$ in table A.4.

\subsubsection{Study cases}

In the first set of simulations, the two built-in models from chapter 7 and the new model from chapter 6 are studied. In the second, one of the models is chosen to test the additional signal 


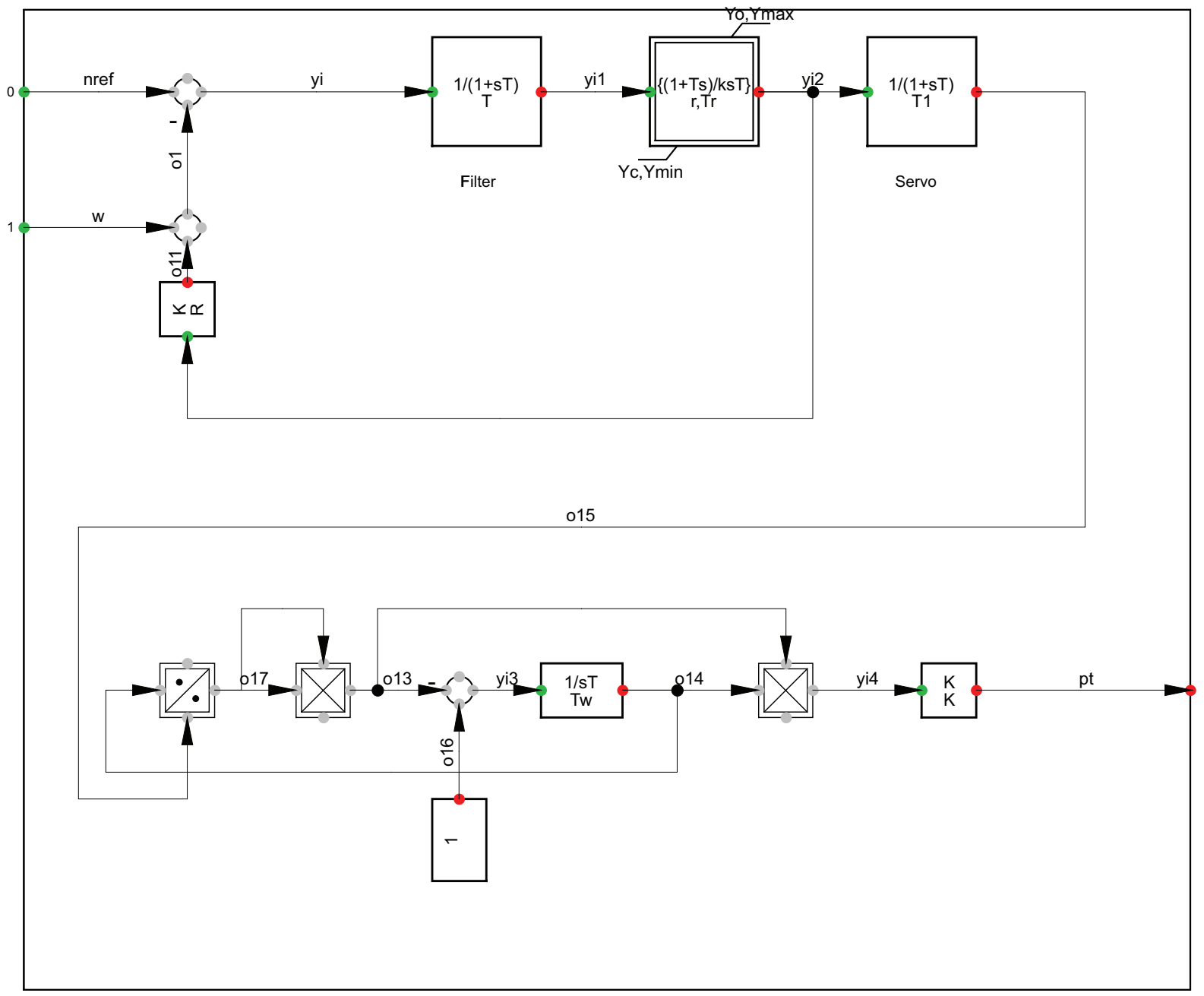

Figure 8.2: Turbine governor in Power Factory

described in section 5.6 , 


\subsection{Model comparison}

In this section, the synchronous generators are equipped with an excitation system with PSS depicted in figure 8.3 and whose values are presented in the appendix $\mathrm{A}$ in table $\mathrm{A} .2$.

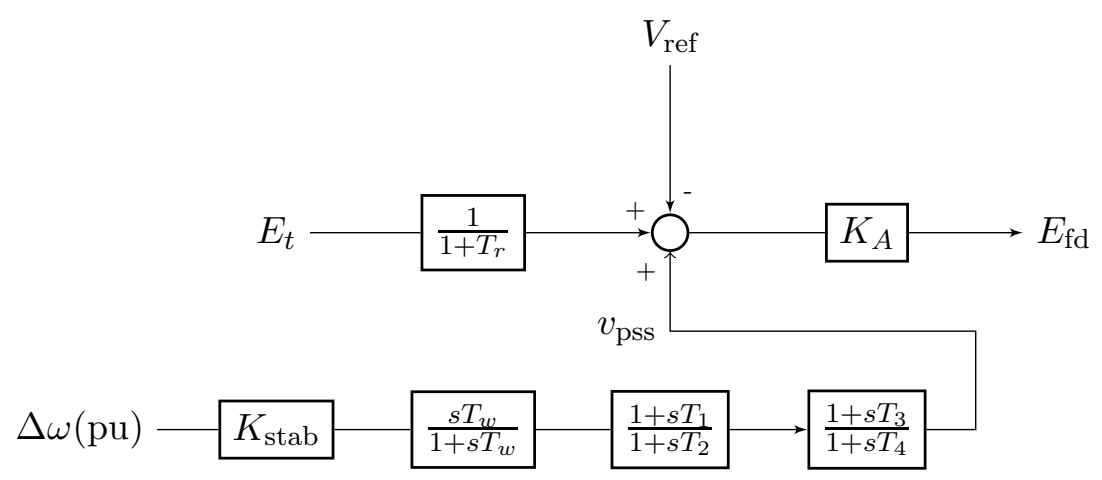

Figure 8.3: Excitation system with PSS

As for the DFIG, active and reactive power are controlled by the scheme described by figure 5.3 with the controllers defined as

$$
\begin{aligned}
& \mathrm{PI}_{p}=K_{p}\left(1+\frac{1}{s T_{d}}\right), \\
& \mathrm{PI}_{q}=K_{q}\left(1+\frac{1}{s T_{q}}\right),
\end{aligned}
$$

and with values in the appendix in table A.5.

The following disturbances have been simulated:

1. disturbance 1: a three-phase short-circuit at bus 9 , occurring at 5 seconds and cleared after 100 milliseconds

2. disturbance 2: same three-phase short-circuit fault but a line is tripped after clearing it

3. disturbance 3: a reactive power setpoint increase up to $0.3 \mathrm{pu}$

\subsubsection{Results}

Results can be found in figures 8.4, 8.5 and 8.6. For disturbances 1 and 2, all models behave in the same way. They have the same dymamics and steady-state values. For disturbance 3 however, Model B differs from the other two, especially in terms of speed and generated active power. 


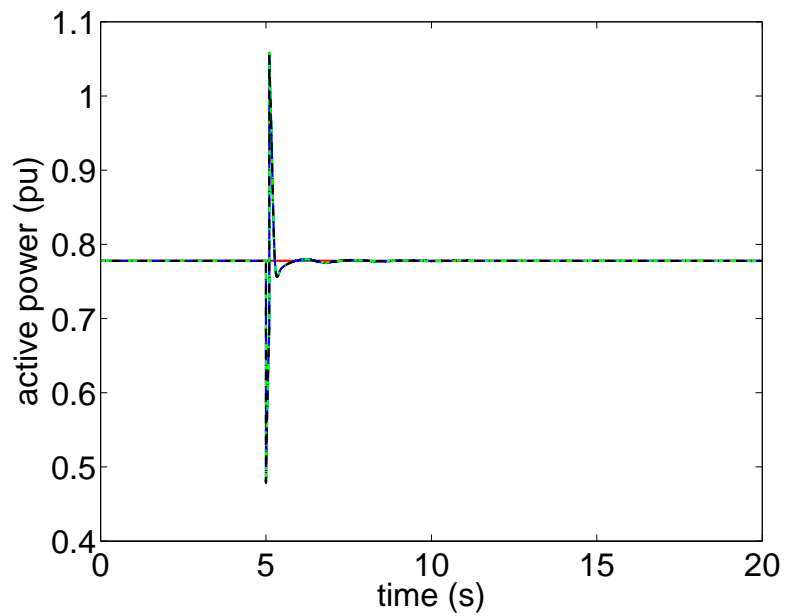

(a) Active Powers

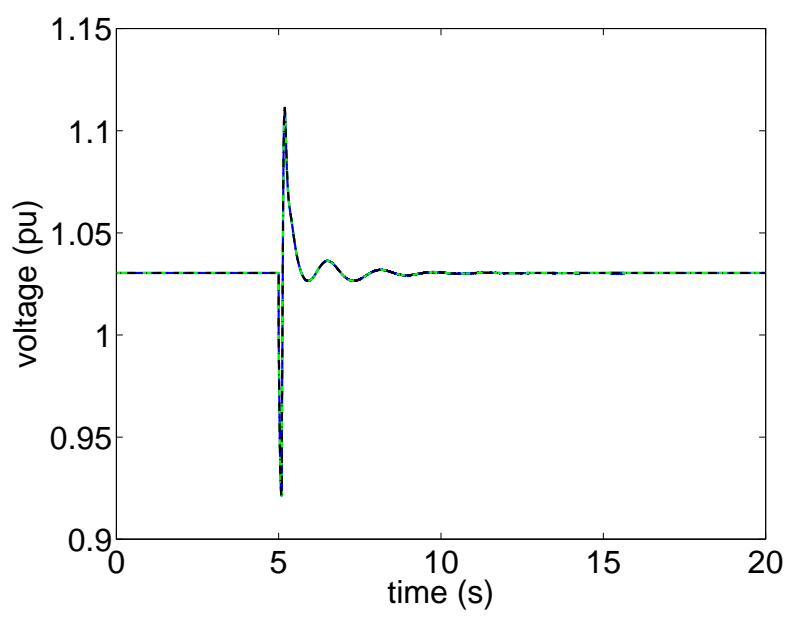

(c) Voltages at bus 1

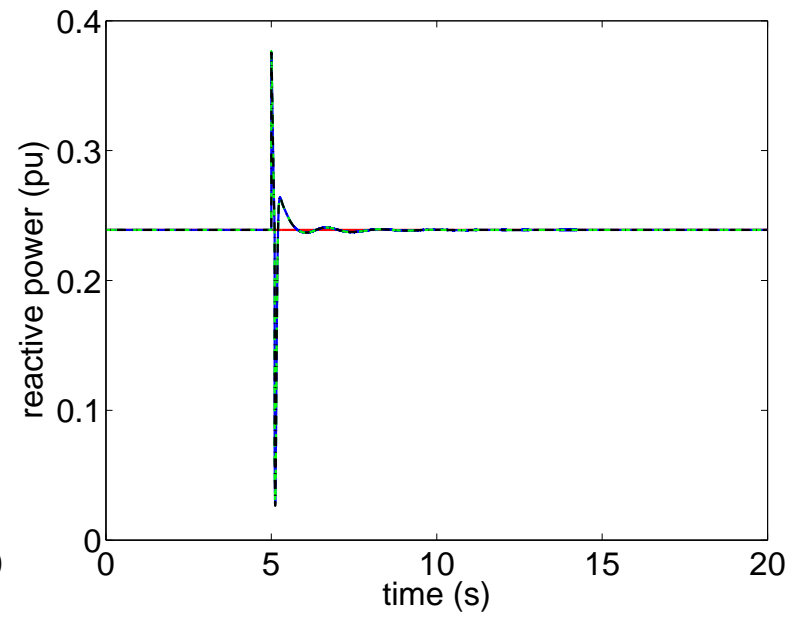

(b) Reactive Powers

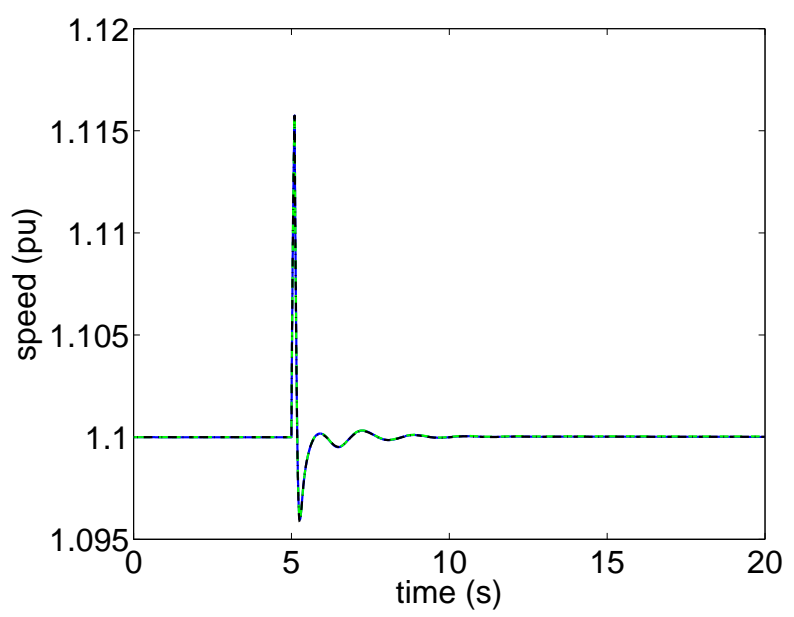

(d) Speeds of the generators

Figure 8.4: Disturbance 1, for Model $C$ [dashed green], Model $A$ [solid blue] and Model B [dashdotted black], setpoints [solid red] 


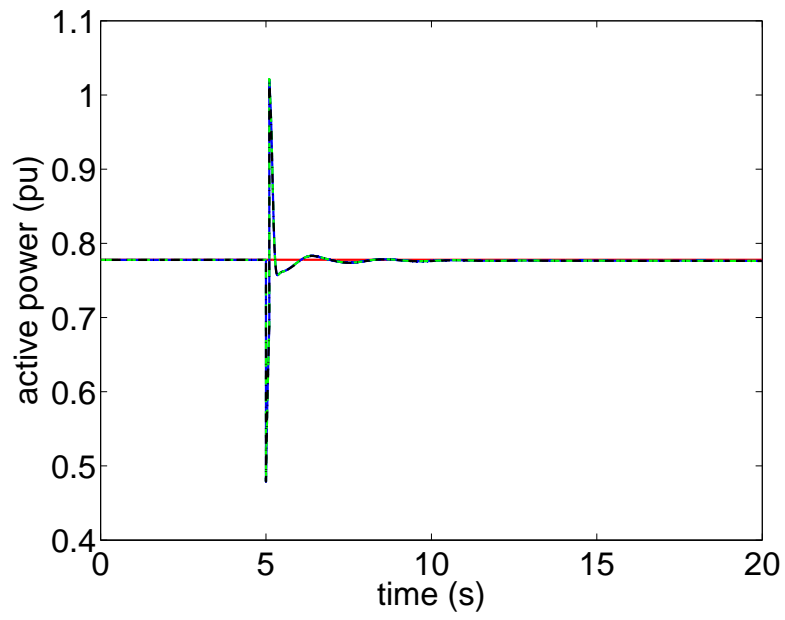

(a) Active Powers

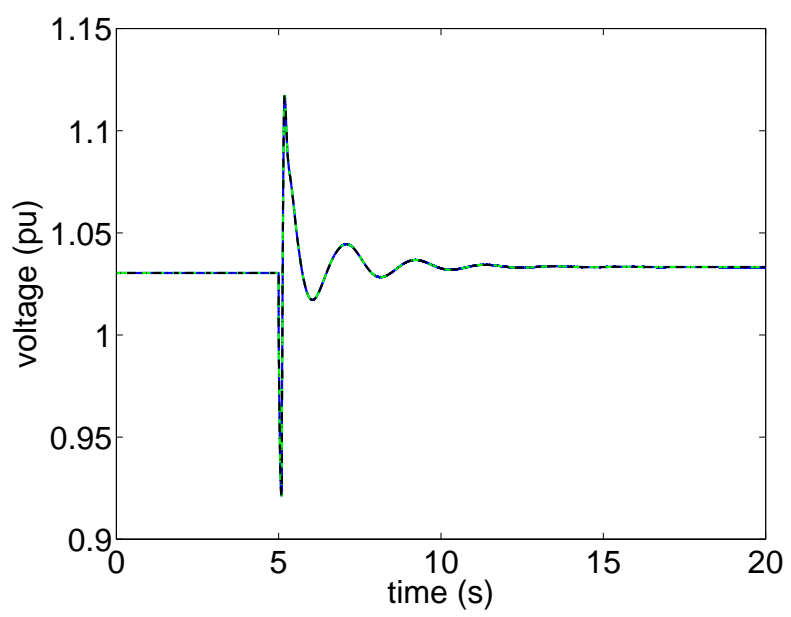

(c) Voltages at bus 1

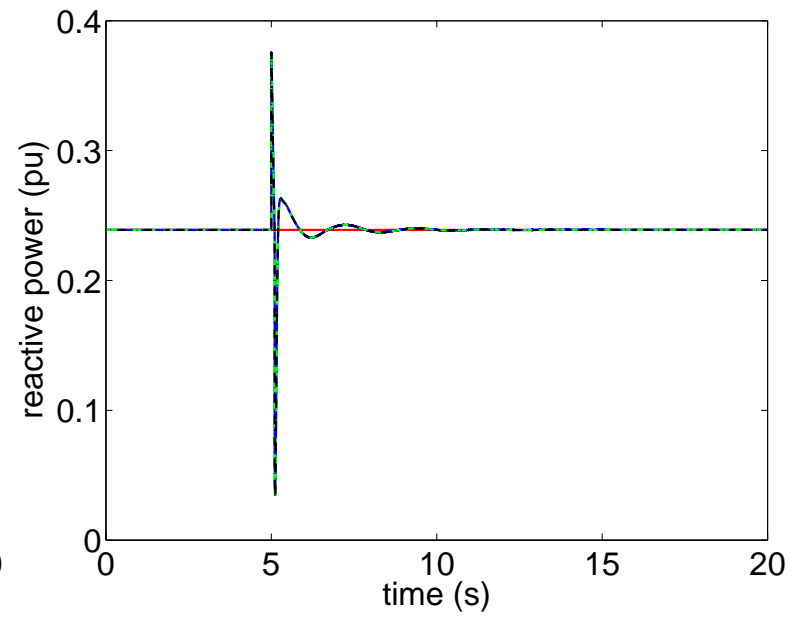

(b) Reactive Powers

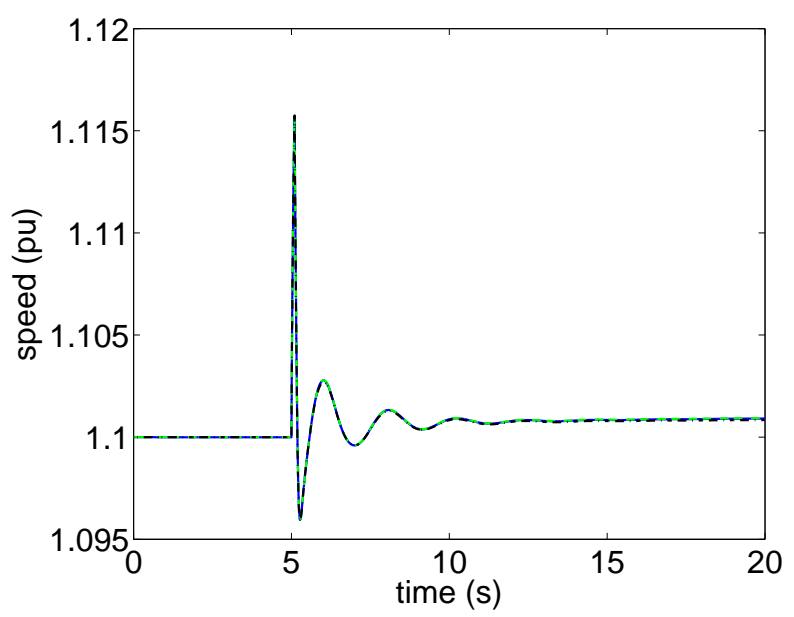

(d) Speeds of the generators

Figure 8.5: Disturbance 2, for Model $C$ [dashed green], Model $A$ [solid blue] and Model B [dashdotted black], setpoints [solid red] 


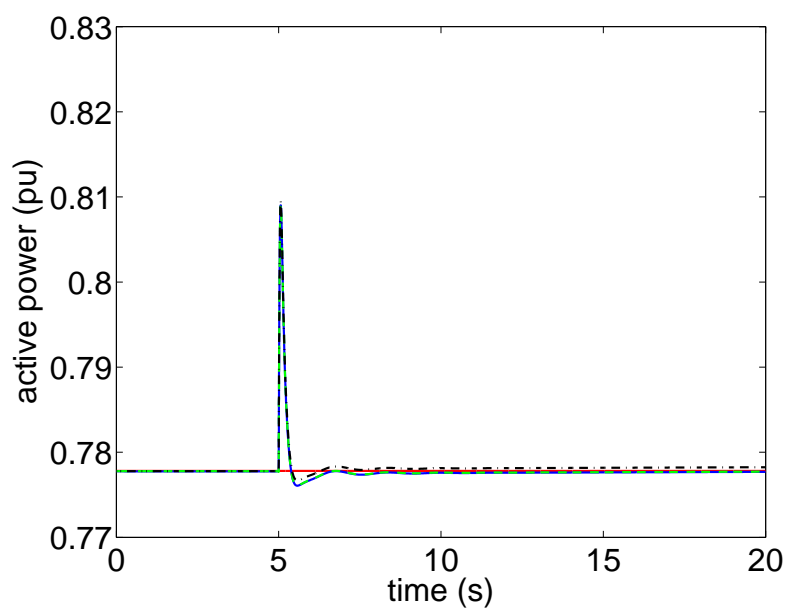

(a) Active Powers

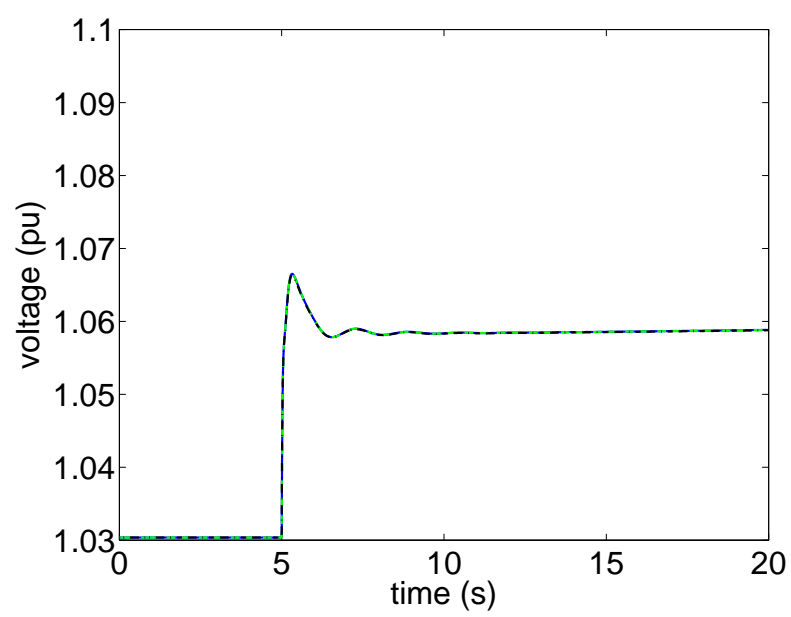

(c) Voltages at bus 1

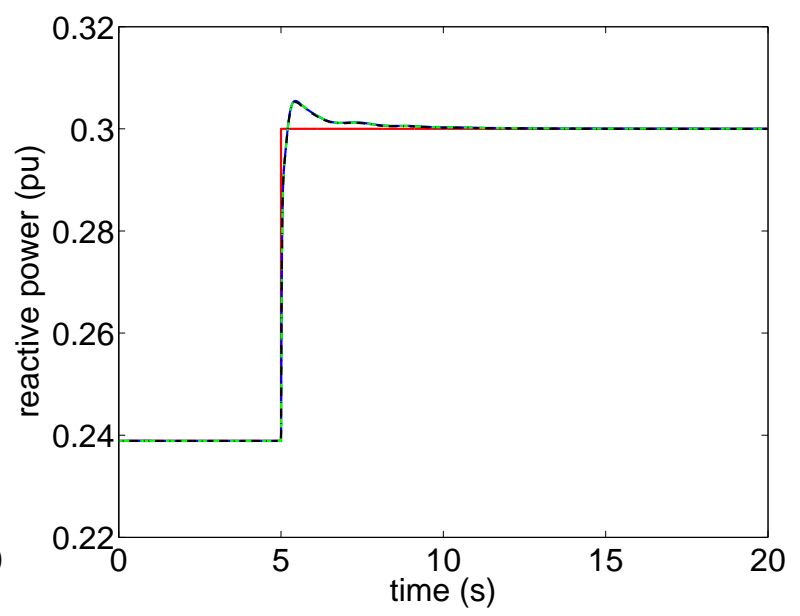

(b) Reactive Powers

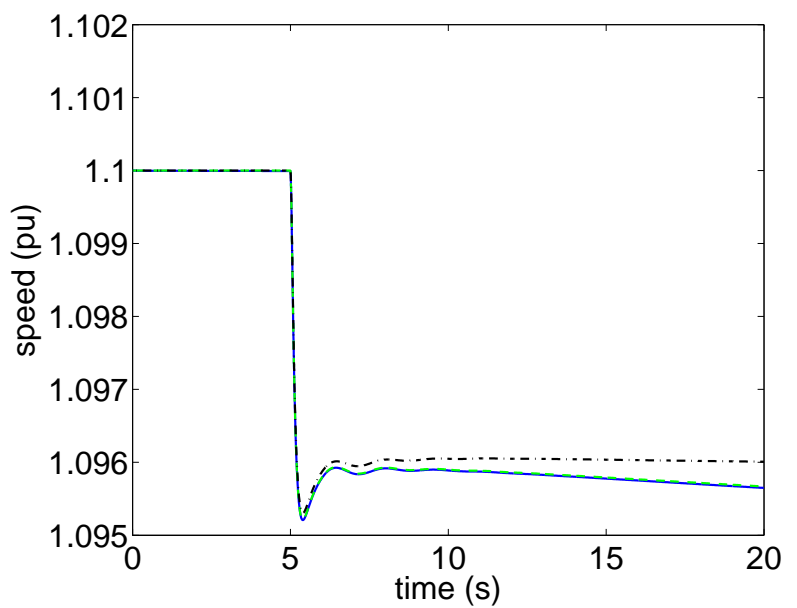

(d) Speeds of the generators

Figure 8.6: Disturbance 3, for Model $C$ [dashed green], Model $A$ [solid blue] and Model B [dashdotted black], setpoints [solid red] 
Magnified parts of these graphs are shown in figures 8.7, 8.8 and 8.9. For disturbance 3, Model $B$ deviates from Model $A$ and Model $C$ mostly during the first oscillation. The differences are very small and concern amplitudes. The frequency of the oscillations are nearly the same. These differences may arise because the models are defined differently during the load flow calculations. The load flow values may therefore be slightly different and those differences may be amplified during the simulations. Another reason could be that small amounts of reactive power may flow between the rotor and the grid with Model B, as explained in section 7 , which would influence the behavior of the machine.

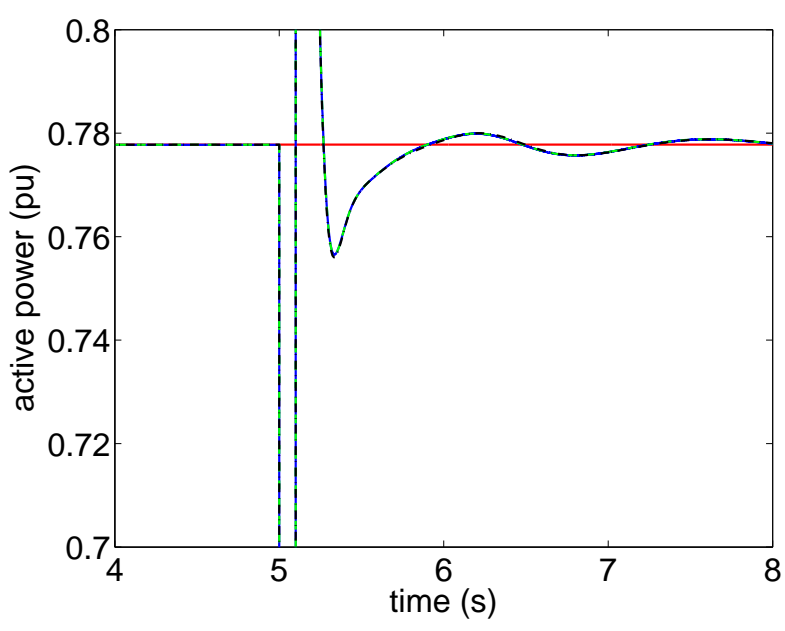

(a) Active Powers

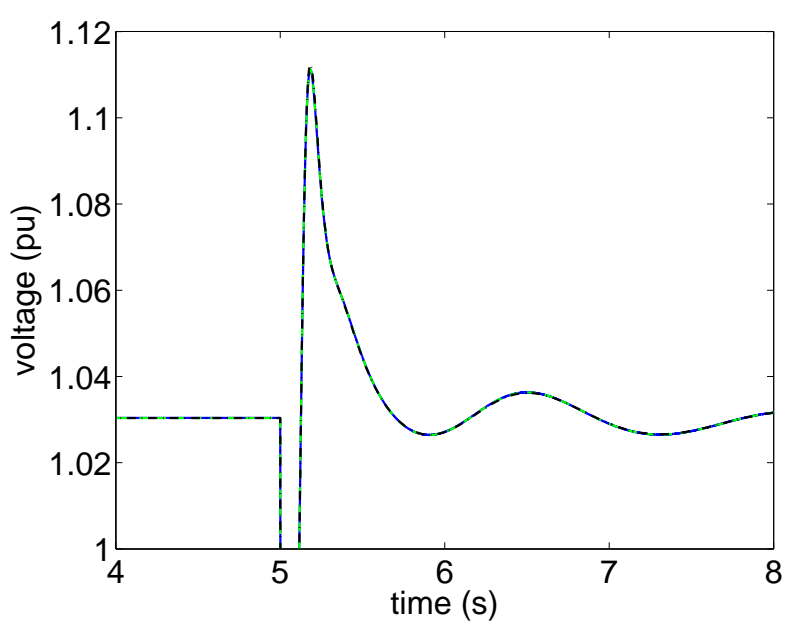

(c) Voltages at bus 1

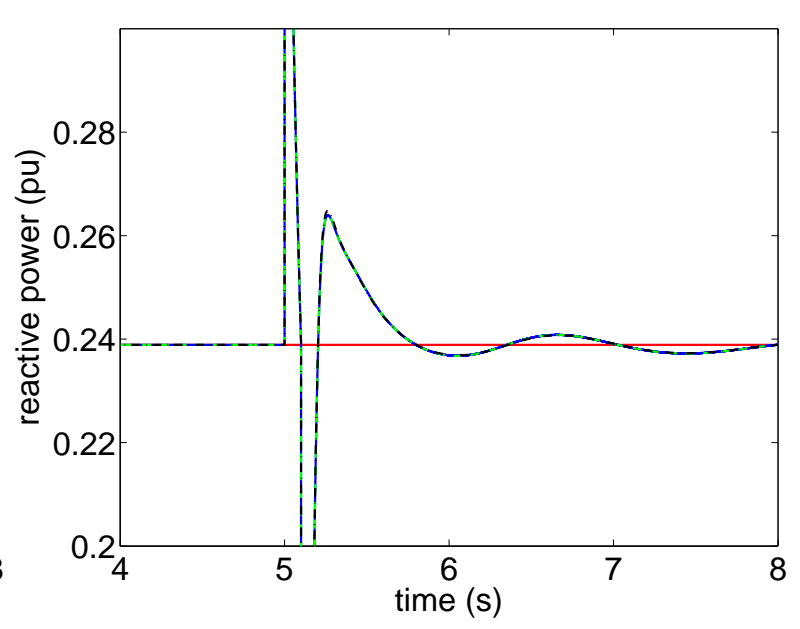

(b) Reactive Powers

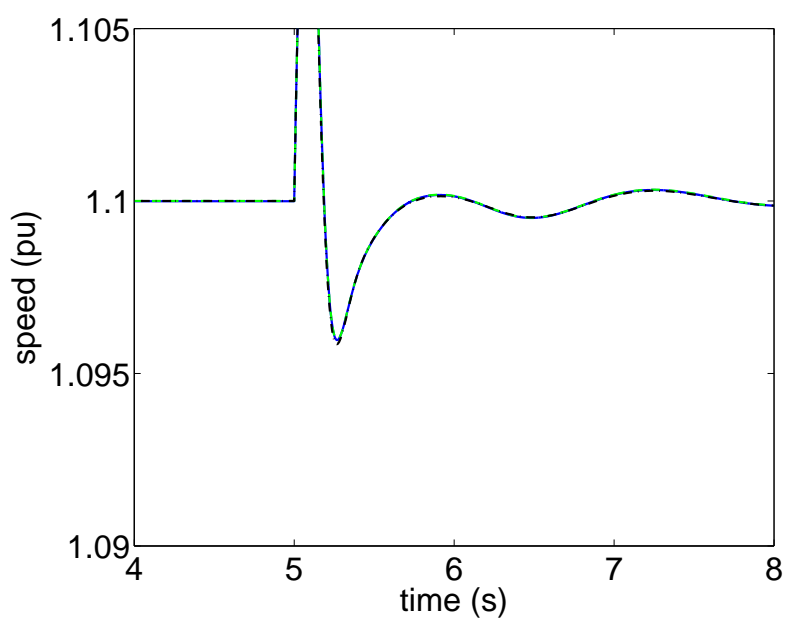

(d) Speeds of the generators

Figure 8.7: Disturbance 1, magnified parts, for Model $C$ [dashed green], Model A [solid blue] and Model $B$ [dashdotted black], setpoints [solid red] 


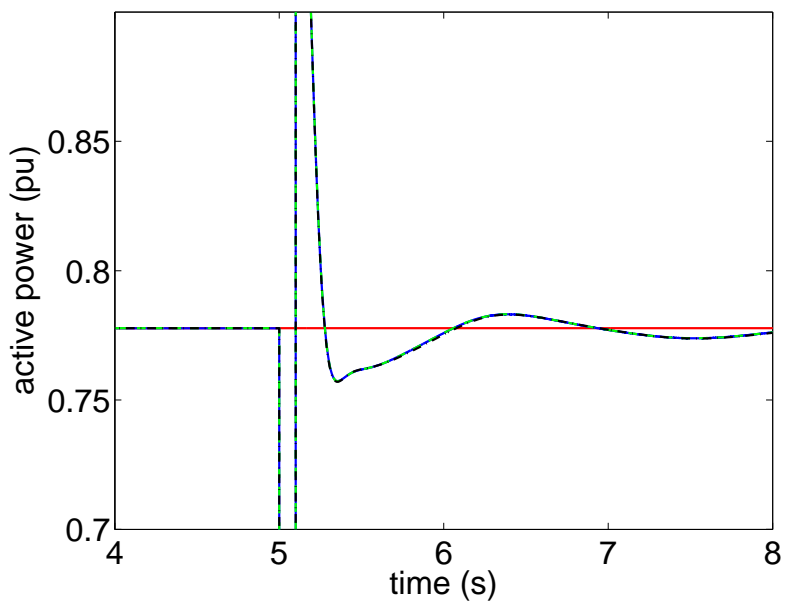

(a) Active Powers

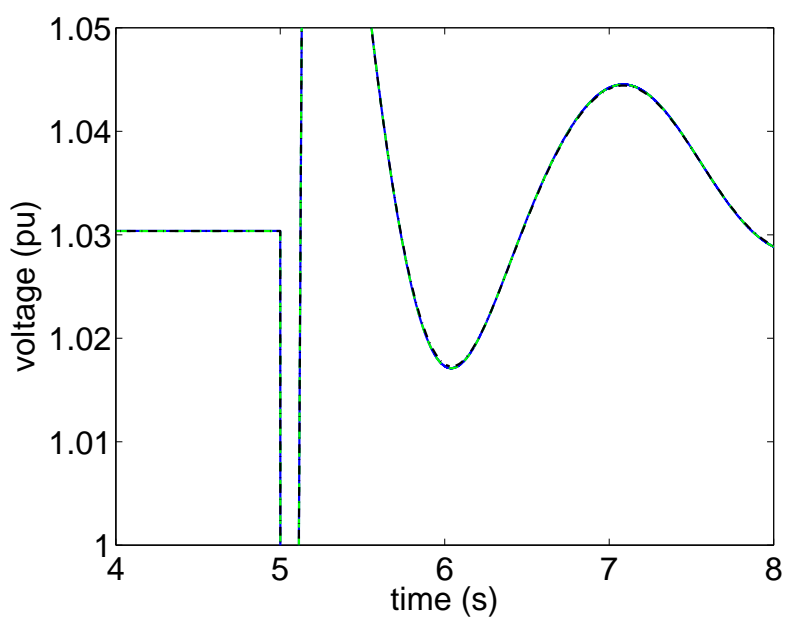

(c) Voltages at bus 1

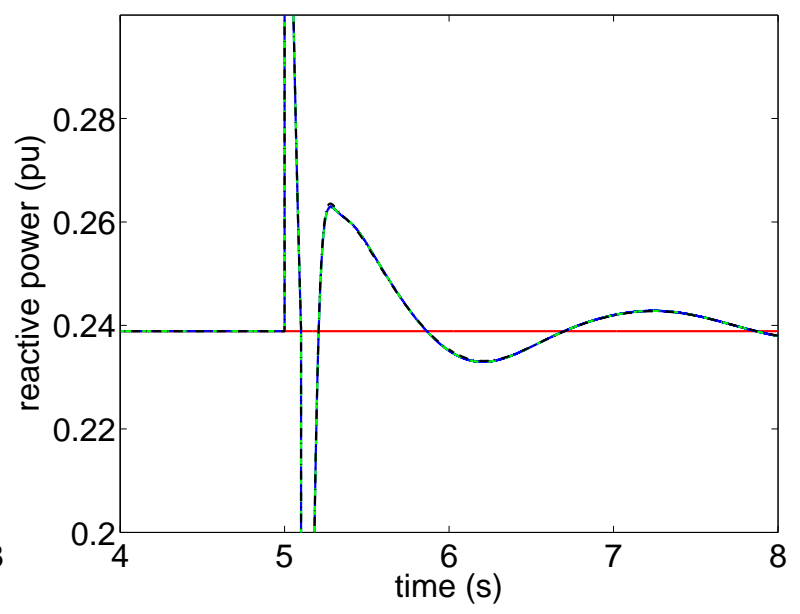

(b) Reactive Powers

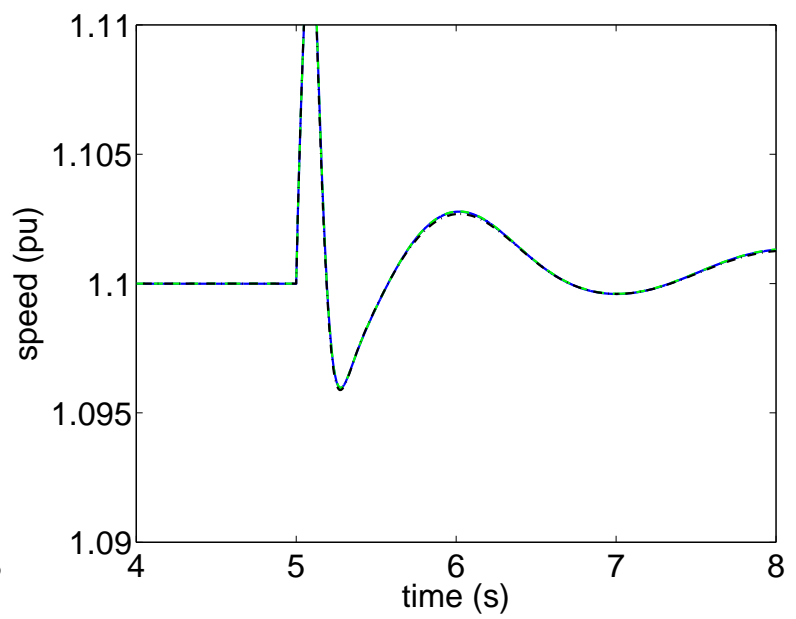

(d) Speeds of the generators

Figure 8.8: Disturbance 2, magnified parts, for Model $C$ [dashed green], Model $A$ [solid blue] and Model B [dashdotted black], setpoints [solid red] 


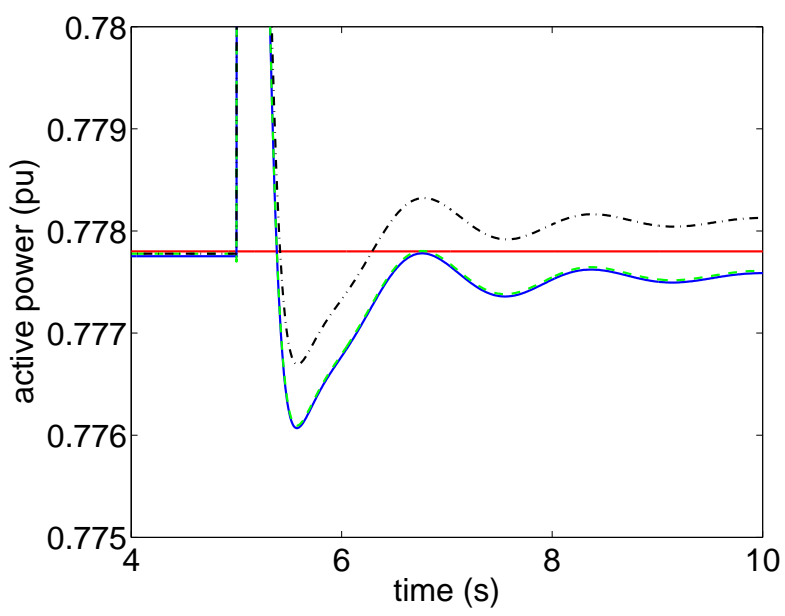

(a) Active Powers

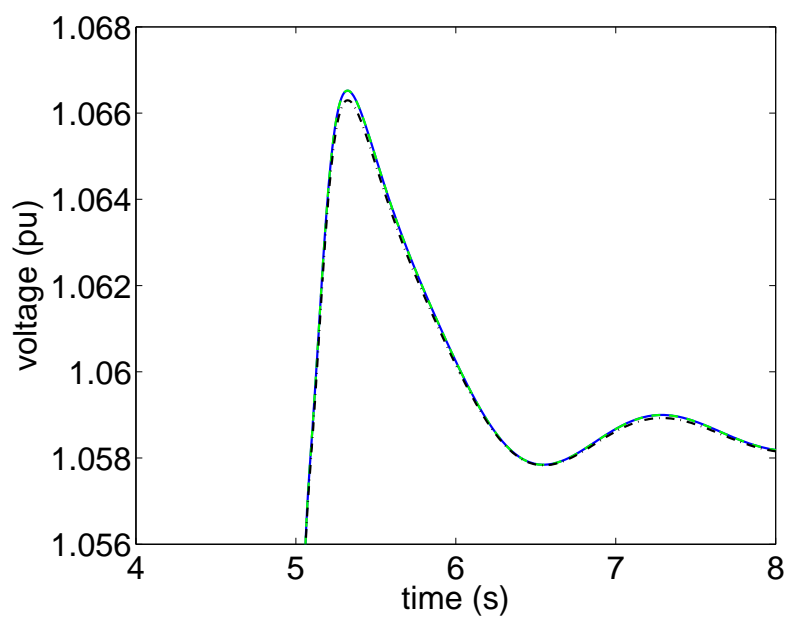

(c) Voltages at bus 1

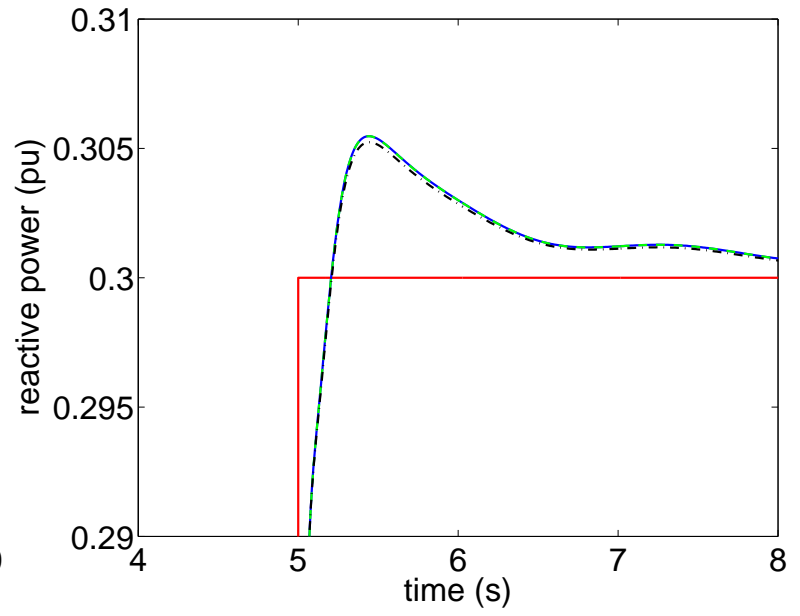

(b) Reactive Powers

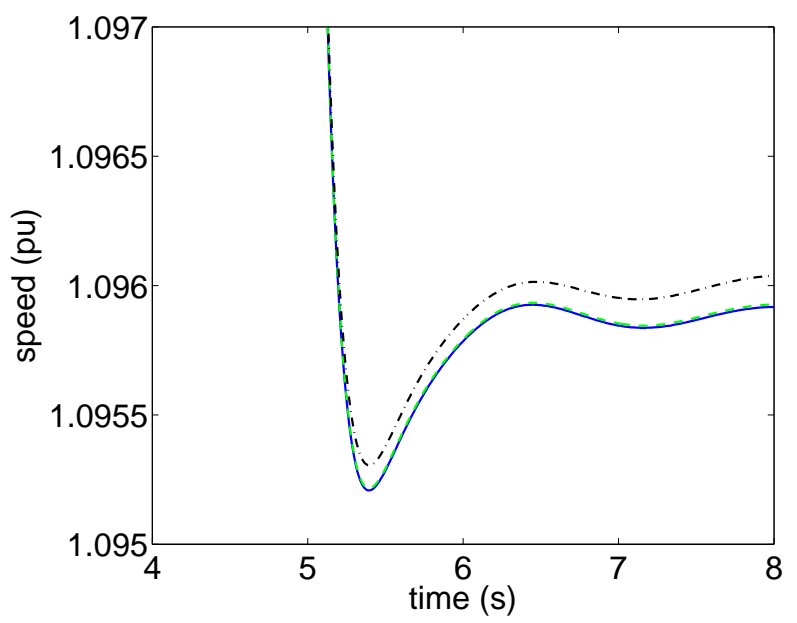

(d) Speeds of the generators

Figure 8.9: Disturbance 3, magnified parts, for Model $C$ [dashed green], Model A [solid blue] and Model B [dashdotted black], setpoints [solid red] 
Table 8.1: Simulation time comparison - elapsed time in seconds

\begin{tabular}{lccc}
\hline & Model $A$ & Model B & Model $C$ \\
\hline Simulation 1 & 55 & 3 & 5 \\
Simulation 2 & 70 & 5 & 8 \\
Simulation 3 & 10 & 3 & 3 \\
\hline
\end{tabular}

\subsubsection{Simulation speed}

Computation time is also an important criterion. A speed comparison has therefore been performed. The elapsed time for each simulation and each model is gathered in table 8.1. The simulation times of Model B and the Model $C$ compared to Model $A$ can be nearly 20 times faster, depending on simulations. This is a considerable difference and makes Model A only interested to use for specific simulations such as studies of the DC-link behavior.

\subsubsection{Comments}

The built-in model termed as Model $B$ stands out in this comparison. It is much faster than the other built-in model and offers the simplicity of a built-in component. It is suited for simulations when the DC-link dynamics are not of interest and when the generator as a whole is studied.

The new model is more complicated to handle but presents more flexibility because it relies on code. It can thus be altered according to users' wishes and can therefore be used when specificities not implemented in the built-in models are studied (used as a first-order model for example).

\subsection{Additional signal}

In this part, the DFIG is Model B (depicted in figure 7.2). The control strategy will be altered to include the additional signal as presented in section 5.6 and compared to the scheme without this extra signal. The system is known for interarea oscillations [9]. Generators G3 and G4 are the critical machines and generator G2 is the non-critical machine. According to section 5.6. the critical and non-critical angles and speeds are defined as:

$$
\begin{aligned}
\delta_{C} & =\frac{H_{3} \delta_{3}+H_{4} \delta_{4}}{H_{3}+H_{4}}, \\
\delta_{N C} & =\delta_{2}, \\
\omega_{C} & =\frac{H_{3} \omega_{3}+H_{4} \omega_{4}}{H_{3}+H_{4}}, \\
\omega_{N C} & =\omega_{2},
\end{aligned}
$$

where $H_{2}$ and $\delta_{2}, H_{3}$ and $\delta_{3}$ and $H_{4}$ and $\delta_{4}$ are the inertias and rotor angles of generators 2,3 and 4 .

Voltage is controlled instead of reactive power. The controllers are therefore defined by:

$$
\begin{aligned}
& \mathrm{PI}_{p}=K_{p}\left(1+\frac{1}{s T_{d}}\right), \\
& \mathrm{PI}_{v}=K_{v}\left(1+\frac{1}{s T_{v}}\right),
\end{aligned}
$$


The values used for this simulation are presented in appendix $\mathrm{A}$ in table A.6.

The excitation system for the synchronous generators is IEEE type AC4, depicted in figure 8.10 from Power Factory, whose parameters are presented in the appendix $\mathrm{A}$ in table A.3.

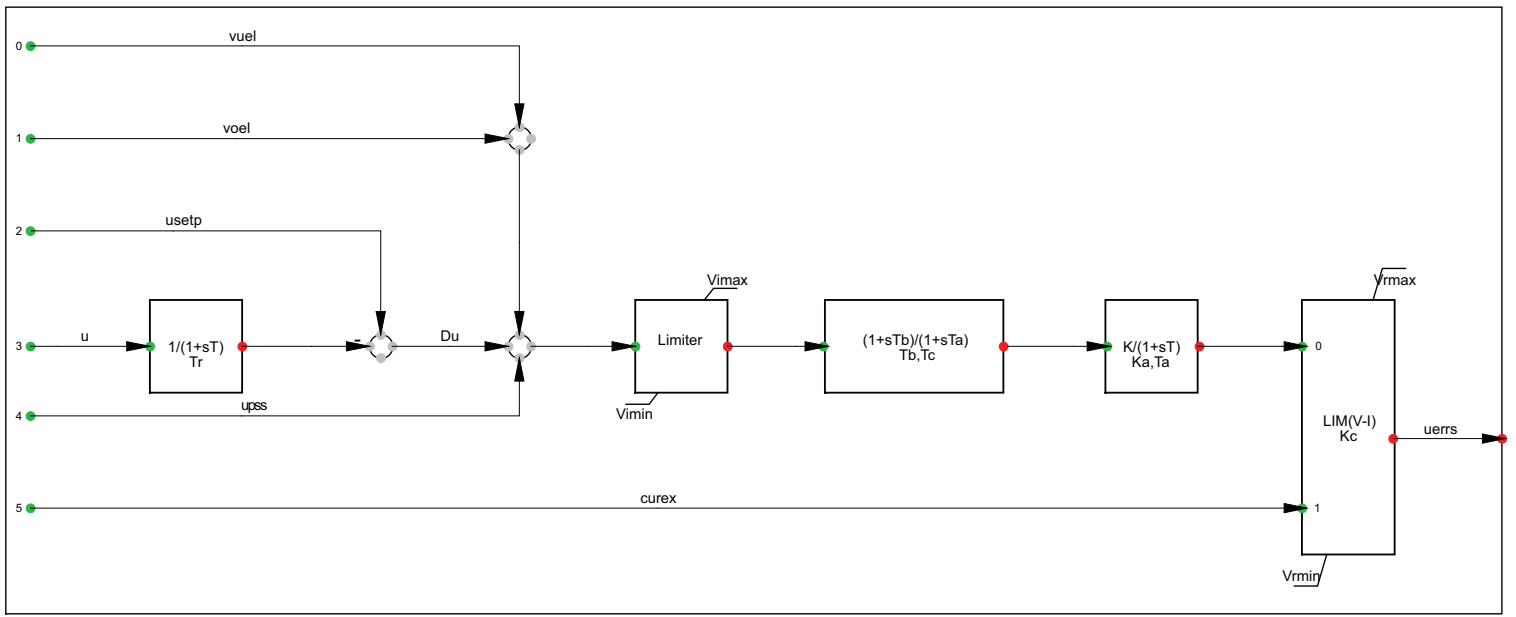

Figure 8.10: Excitation system IEEE type AC4

The PSS have been taken away from the synchronous generators. The aim is to study whether the so-called SIME signal can add damping to power oscillations. The following simulations have been carried out:

1. disturbance 1: a three-phase short circuit at bus 8 , occurring at 5 seconds and cleared after 100 milliseconds

2. disturbance 2: the same fault but a line between bus 8 and 9 is tripped after the fault is cleared

3. disturbance 3: a decrease of the active power setpoint by $5 \%$

4. disturbance 4: a decrease of the voltage setpoint by $8 \%$

In figures 8.11 and 8.12 , the results from disturbances 1 and 2 are shown. For both disturbances, the generated active and reactive powers, the voltages at buses 1 and 8 and the active power along one line between buses 8 and 9 have been plotted.

Without the SIME signal $y_{\text {sime }}$ from section [5.6, the system becomes unstable. With the SIME signal, the system is stable. The oscillations are however poorly damped in the first simulation. The SIME signal adds much more damping in the system for disturbance 2 .

This is reflected by both the local (generated powers and voltage at bus 1) and remote (active power along one line between buses 8 and 9) quantities. 


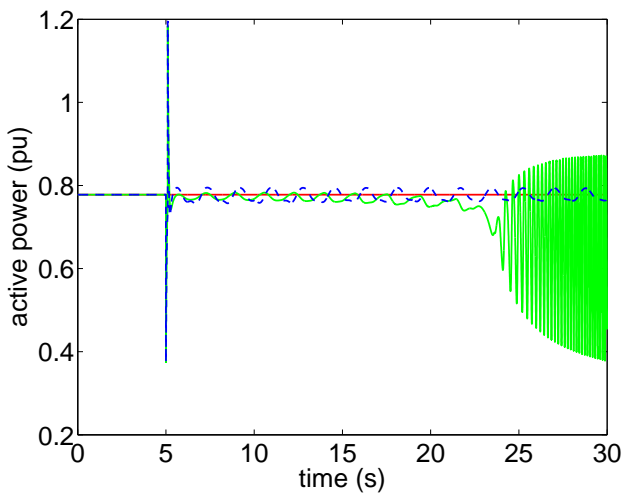

(a) Active Powers

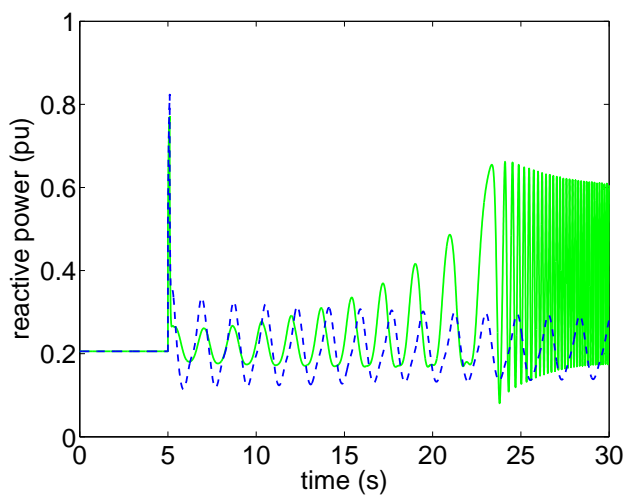

(c) Reactive powers

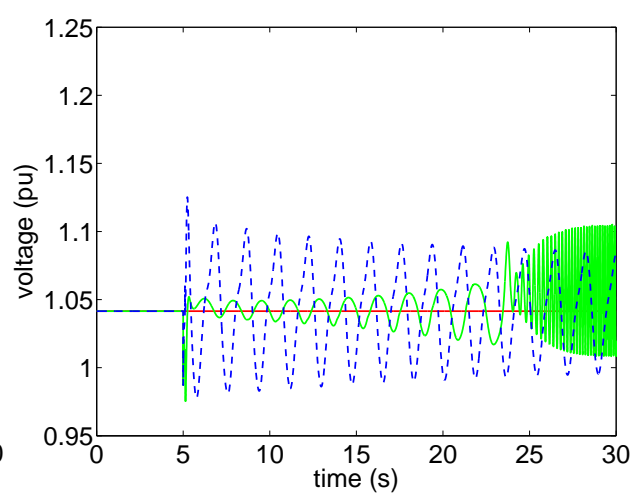

(b) Voltages at bus 1

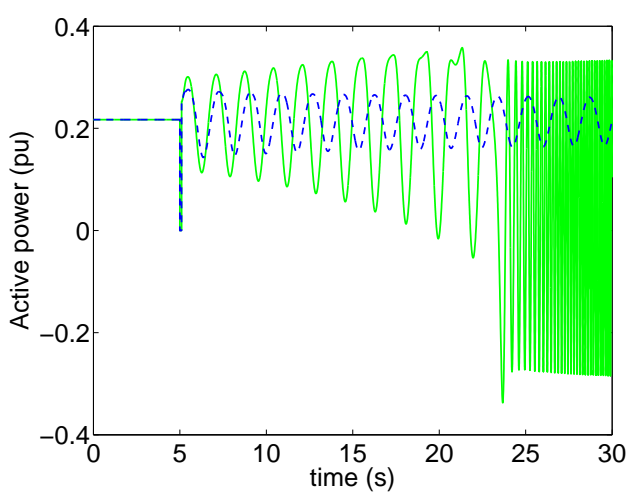

(d) Power along a line between buses 8 and 9

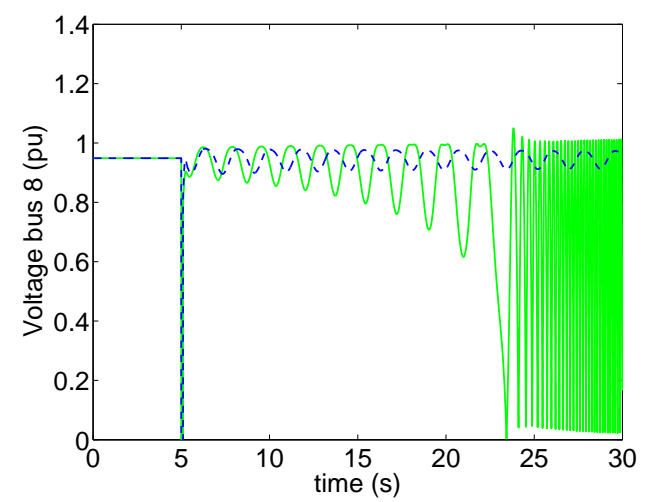

(e) Voltage at bus 8

Figure 8.11: Disturbance 1, with [dashed blue] and without [solid green] the additional signal 


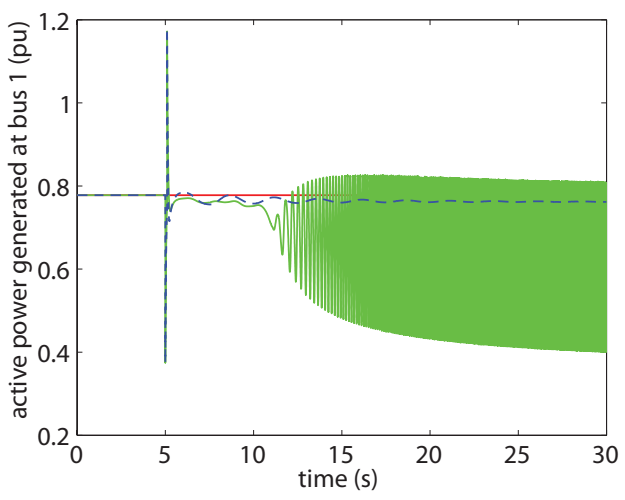

(a) Active Powers

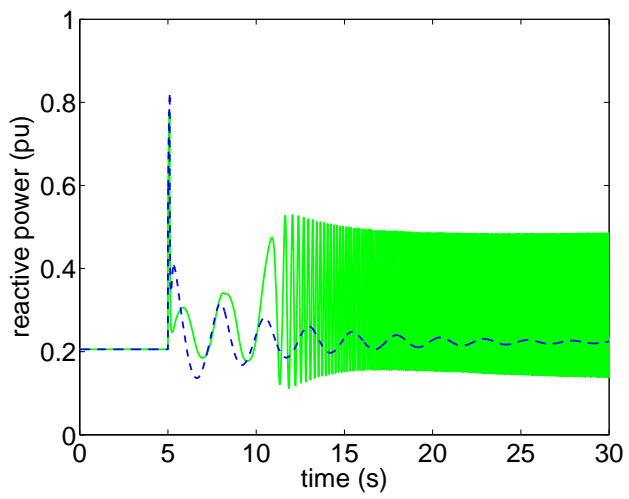

(c) Reactive powers

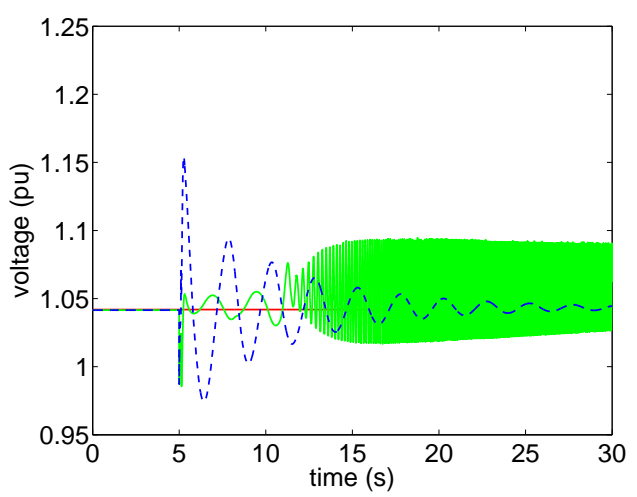

(b) Voltages at bus 1

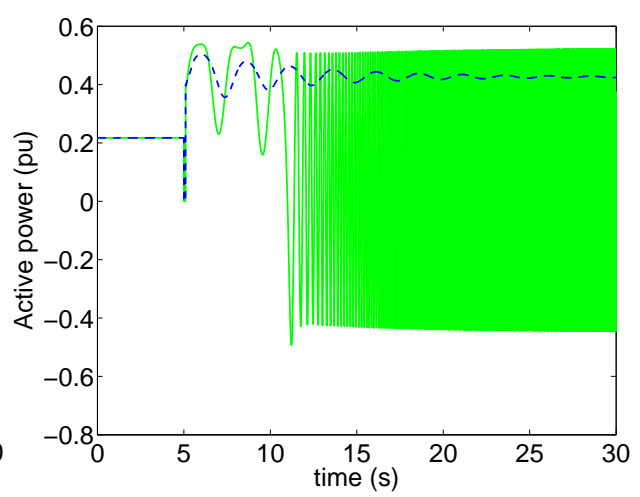

(d) Power along a line between buses 8 and 9

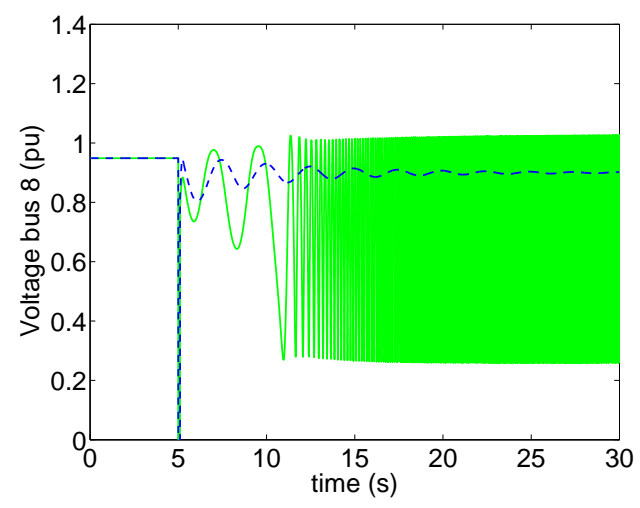

(e) Voltage at bus 8

Figure 8.12: Disturbance 2, with [dashed blue] and without [solid green] the additional signal 
Without the SIME signal, the frequency of the oscillations is very high and tend to point to something more than unstable oscillations. This behavior comes from the voltage regulators with which the synchronous generators are equipped. In table A.3, it can be noticed that some parameters are limiting parameters. In particular, $V_{\text {rmin }}$ and $V_{\text {rmax }}$ are used to limit the output of the regulators. In figures 8.13 and 8.14 , these outputs are plotted. It can be observed that in both cases, the voltage regulator at generator 2 hits the limit after the fault. Without the SIME signal, unstable oscillations arise and the three voltage regulators hit the limit in the end. With the SIME signal, the three voltage regulators go back to their setpoint values.

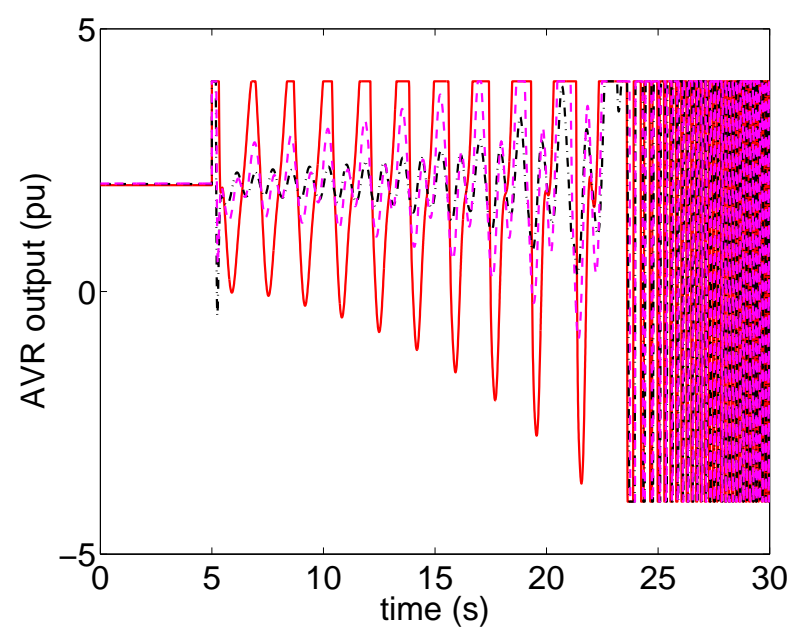

(a) Without SIME signal

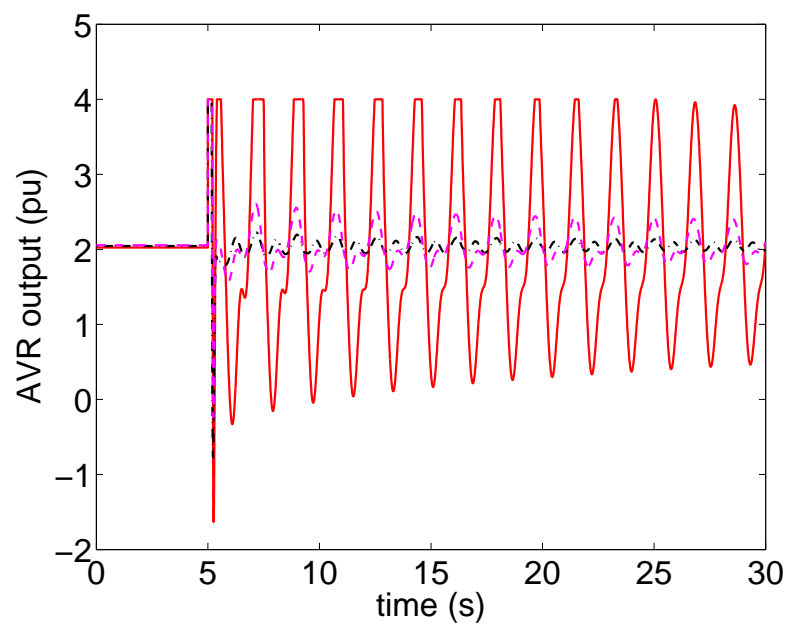

(b) With SIME signal

Figure 8.13: Disturbance 1, outputs of the voltage regulators, generator 2 [solid red], generator 3 [dashdotted black] and generator 4 [dotted magenta] 


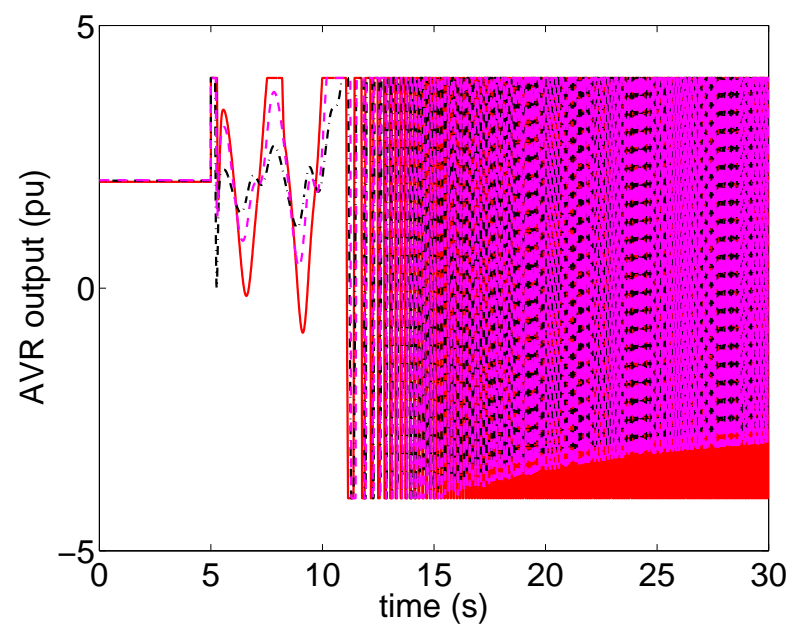

(a) Without SIME signal

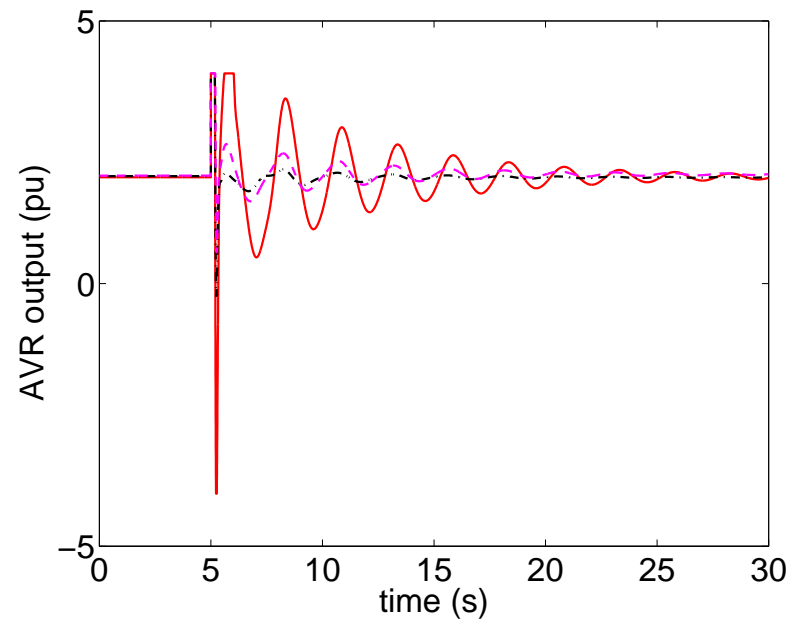

(b) With SIME signal

Figure 8.14: Disturbance 2, outputs of the voltage regulators, generator 2 [solid red], generator 3 [dashdotted black] and generator 4 [dotted magenta] 
Responses to disturbances 3 and 4 are plotted in figures 8.15] and 8.16. The SIME signal improves the damping for these disturbances as well. One interesting fact to notice with disturbance 3 is that the local quantities can be misleading. Indeed, if one observes only the generated powers and the voltage at bus 1 , the system has been stabilized by the SIME signal but it seems undamped. However, if remote quantities (power along one line between buses 8 and 9 and voltage at bus 8 ) are observed, the effect of the signal is much more obvious. Without the SIME signal, the system is clearly unstable with growing oscillations while using the SIME signal adds good damping to the system.

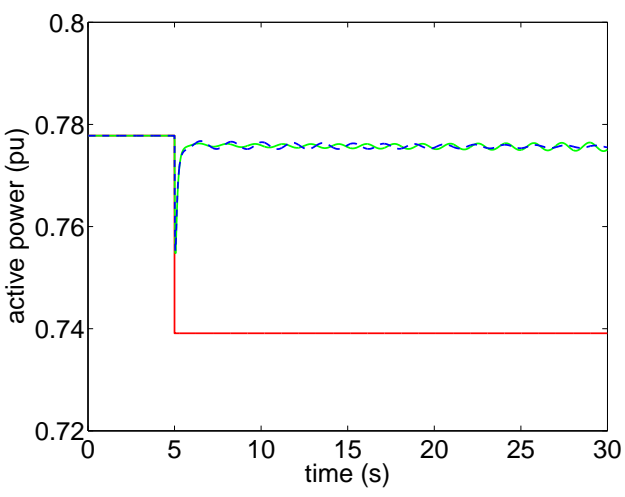

(a) Active Powers

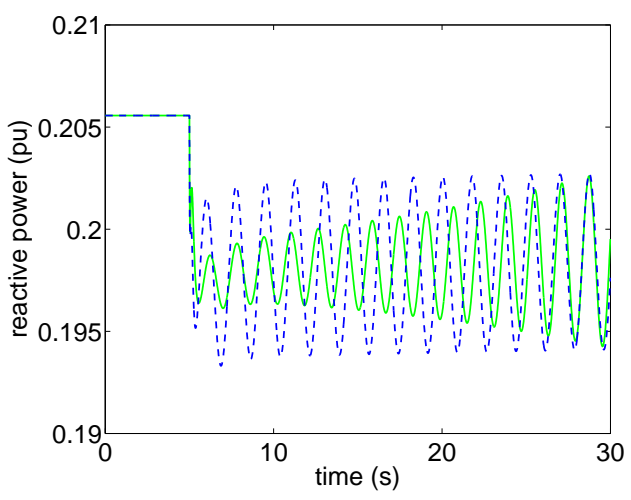

(c) Reactive Powers

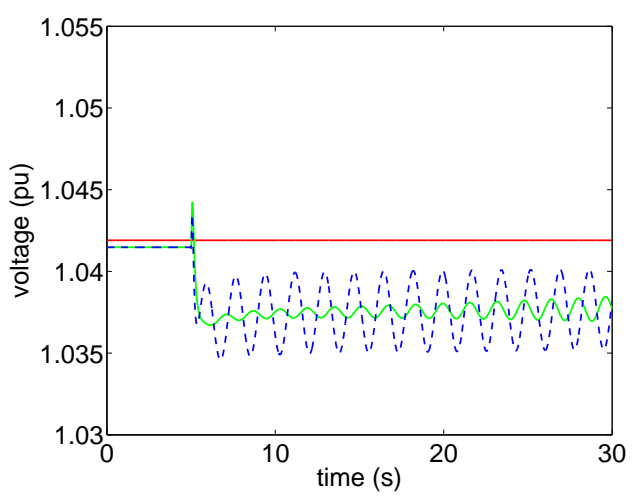

(b) Voltages at bus 1

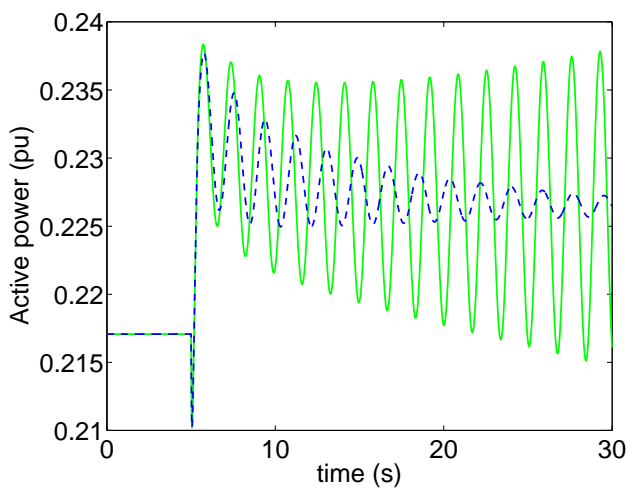

(d) Power along a line between buses 8 and 9

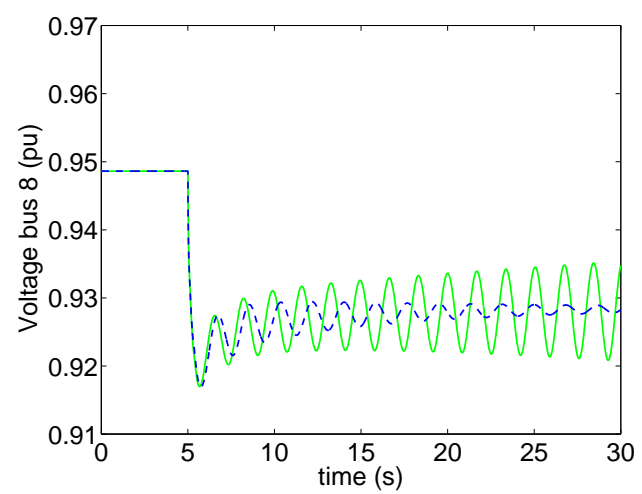

(e) Voltage at bus 8

Figure 8.15: Disturbance 3, with [dashed blue] and without [solid green] the additional signal 


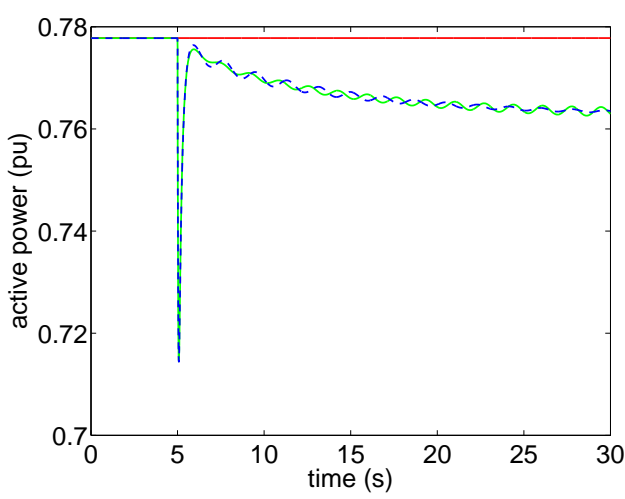

(a) Active Powers

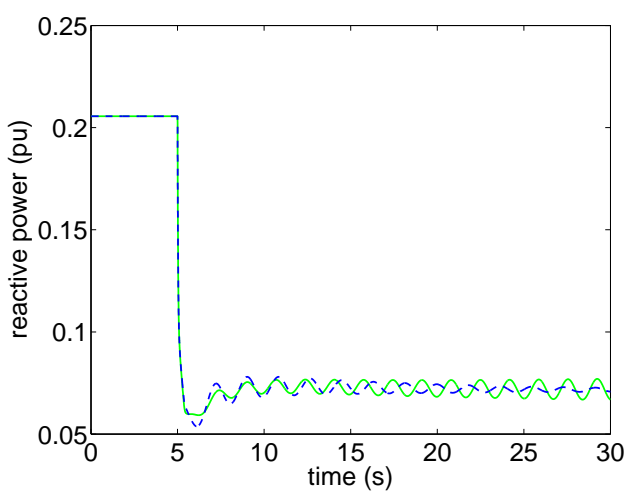

(c) Reactive Powers

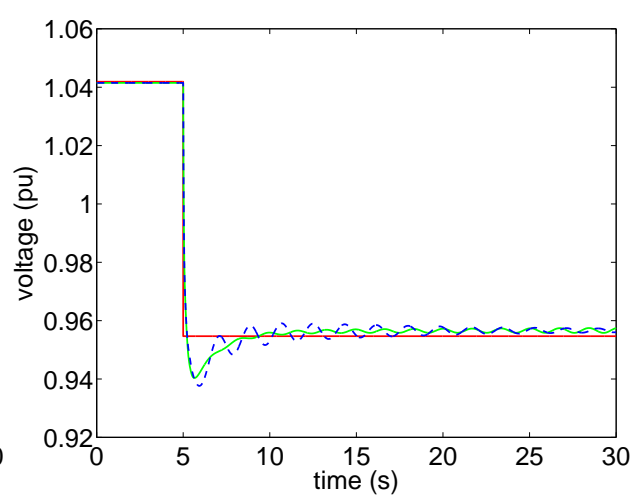

(b) Voltages at bus 1

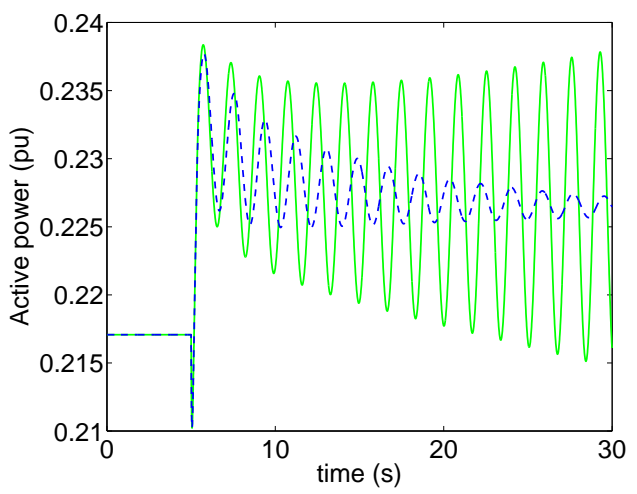

(d) Power along a line between buses 8 and 9

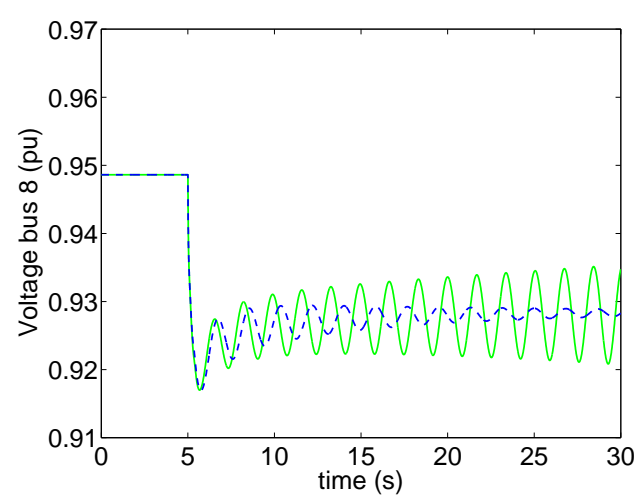

(e) Voltage at bus 8

Figure 8.16: Disturbance 4, with [dashed blue] and without [solid green] the additional signal 



\section{Chapter 9}

\section{Conclusion}

This work has been the opportunity to gather knowledge about doubly-fed induction generators. The theory lying behind these machines has been used to create a new model, which has been implemented in Power Factory. This model and two built-in models were compared in this study.

Model $B$ offers many more possibilities for load flow, can be defined either as a PQ or a PV bus and can be controlled to set the load flow values at a remote bus. Also, the difference in speed with Model $A$ is very large. Considerable time can be gained by using this model in simulations. Finally, it is simpler to use and comes as a all-in-one component whereas the other model comes with a DC link, another converter and a transformer for the rotor side. This makes Model $A$ suitable only when modeling the converters is of interest.

The new model that was developed has been tested just in a few cases in this study. It is more complicated to use than built-in components but it is more flexible because it relies on code and can be made to do exactly what the user wishes.

The SIME signal has shown great potential to add damping to the system when using DFIG.

We propose therefore a follow-up to this work. It may be interesting to use the new model more extensively and test it in many different systems. An interesting study would be to use the flexibility of the model to take it down to a first order model as described in the theory part of this work. Insightful results could also be obtained by taking it to a fifth-order model and comparing it with the models of same order in Power Factory. 

Appendices 



\section{Appendix A}

\section{Values used in the simulation}

The values for the governors are presented in table A.1 and those for the DFIG in table A.4.

For the model comparison in section 8.2 , the values for the excitation system with PSS depicted in figure 8.3 are presented in table A.2. The values for the PI controllers can be found in table A.5.

For the SIME signal study in section 8.3, another excitation system, depicted in figure 8.10, was used. The values are presented in table A.3. The values for the PI controllers can be found in table A.6.

Table A.1: Parameters of the governors

\begin{tabular}{cccc}
\hline Parameter & Value & Parameter & Value \\
\hline $\mathrm{T}$ & $0.05(\mathrm{~s})$ & $T_{r}$ & $5(\mathrm{~s})$ \\
$T_{1}$ & $0.2(\mathrm{~s})$ & $Y_{c}$ & 0 (p.u.) \\
$T_{w}$ & 1 (s) & $Y_{\min }$ & 0 (p.u.) \\
$\mathrm{K}$ & 1 (p.u.) & $Y_{0}$ & 0.1 (p.u.) \\
$\mathrm{R}$ & 0.04 (p.u.) & $Y_{\max }$ & 2 (p.u.) \\
$\mathrm{r}$ & 0.8 (p.u.) & & \\
\hline
\end{tabular}

Table A.2: Values of the parameters for the excitation system used in the model comparison

\begin{tabular}{cccc}
\hline Parameter & Value & Parameter & Value \\
\hline$K_{a}$ & $200($ p.u. $)$ & $T_{r}$ & $0.01(\mathrm{~s})$ \\
$T_{w}$ & $10(\mathrm{~s})$ & $T_{1}$ & $0.05(\mathrm{~s})$ \\
$T_{2}$ & $0.02(\mathrm{~s})$ & $T_{3}$ & $3(\mathrm{~s})$ \\
$T_{4}$ & $5.4(\mathrm{~s})$ & $K_{\text {stab }}$ & 20 (p.u.) \\
\hline
\end{tabular}


Table A.3: Parameter values for the excitation system in the SIME signal study

\begin{tabular}{cccc}
\hline Parameter & Value & Parameter & Value \\
\hline$T_{r}$ & $0(\mathrm{~s})$ & $K_{a}$ & 300 (p.u.) \\
$T_{b}$ & $0(\mathrm{~s})$ & $T_{a}$ & 0.01 (p.u.) \\
$T_{c}$ & $0(\mathrm{~s})$ & $K_{c}$ & 0 (p.u.) \\
$V_{\text {imin }}$ & $-\infty$ & $V_{\text {imax }}$ & $\infty$ \\
$V_{\text {rmin }}$ & -4 (p.u.) & $V_{\text {rmax }}$ & 4 \\
\hline
\end{tabular}

Table A.4: DFIG parameters

\begin{tabular}{cc}
\hline Name & Value \\
\hline Rated Power & $900(\mathrm{MW})$ \\
Rated Voltage & $3.3(\mathrm{kV})$ \\
Number of poles & 2 \\
Nominal frequency & $50(\mathrm{~Hz})$ \\
Stator resistance $R_{s}$ & 0 (p.u.) \\
Stator reactance $X_{s}$ & 3.1 (p.u.) \\
Rotor resistance $R_{r}$ & 0.01 (p.u.) \\
Rotor reactance $X_{r}$ & 3.1 (p.u.) \\
Mutual reactance $X_{m}$ & 0.1 (p.u.) \\
Acceleration time constant $T_{\mathrm{ag}}$ & $1.145767(\mathrm{~s})$ \\
DC Voltage & 1.3225 (p.u.) \\
\hline
\end{tabular}

Table A.5: Values used in the control scheme for the model comparison

\begin{tabular}{cc}
\hline Parameter & Value \\
\hline$K_{p}$ & 0.2 (p.u.) \\
$T_{p}$ & 2 (s) \\
$K_{q}$ & 0.2 (p.u.) \\
$T_{q}$ & 2 (s) \\
$K_{S p}$ & 0 (p.u.) \\
$K_{S q}$ & 0 (p.u.) \\
\hline
\end{tabular}

Table A.6: Values used in the control scheme with SIME signal

\begin{tabular}{cc}
\hline Parameter & Value \\
\hline$K_{p}$ & 0.1 (p.u.) \\
$T_{p}$ & 2 (s) \\
$K_{v}$ & 0.1 (p.u.) \\
$T_{v}$ & 2 (s) \\
$K_{S p}$ & -10 (p.u.) \\
$K_{S v}$ & 5 (p.u.) \\
\hline
\end{tabular}




\section{Appendix B}

\section{PowerFactory details}

In this section subtleties about Power Factory that may be misleading are gathered.

It may be interesting to know which signals are available for a given components. One way is to dig into the documentation. This is of course acceptable as long as there is some technical reference for the given component, which is not always the case. In our work for example, no documentation for the controlled voltage source exists in the installed version. To know which signals are available, the user must start as if defining a variable set to store result variables. There, in the window that popups, a list with all the accessible values is printed. All signals, calculation parameters, input parameters and state variables can be found in this list.

Finally, a word about the Phase Measurement Device (so-called PLL in Power Factory). This device is used to measure the angle, or frequency, of the voltage at a specific bus. The user must carefully monitor the output values (angle or frequency) since they can be wrong. If it is the case, one way to solve the problem is to increase the gain in the RMS-simulation tab of the PLL. The component should after that follow the angle or frequency variations without difficulty. 



\section{Bibliography}

[1] Wind map 2008. Technical report, European Wind Energy Association, December 2008.

[2] T. Ackermann and L. Söder. An overview of wind energy-status 2002. Renewable and Sustainable Energy Reviews, 6(1-2):67-127, 2002.

[3] DigSilent. Digsilent gmbh, March 2010.

[4] Katherine Elkington. Modelling and control of doubly fed induction generators in power systems. Technical Report TRITA-EE 2009:011, Royal Institute of Technology (KTH), April 2009. Licentiate Thesis.

[5] M. Ghandhari, G. Andersson, M. Pavella, and D. Ernst. A control strategy for controllable series capacitor in electric power systems. Automatica, 37(10):1575 - 1583, 2001. ISSN 0005-1098.

[6] A.D. Hansen, F. Iov, P.E. Sørensen, N.A. Cutululis, C. Jauch, and F. Blaabjerg. Dynamic wind turbine models in power system simulation tool DIgSILENT. Risø National Laboratory for Sustainable Energy, 2007.

[7] P. C. Krause, F. Nozari, T. L. Skvarenina, and D. W. Olive. The theory of neglecting stator transients. IEEE Transactions on Power Apparatus and Systems, 98(1):141-148, January 1979. ISSN 0018-9510.

[8] P.C. Krause, O. Wasynczuk, and S.D. Sudhoff. Analysis of electric machinery. McGraw-Hill New York, 1986.

[9] P. Kundur, N.J. Balu, and M.G. Lauby. Power system stability and control. McGraw-Hill Professional, 1994.

[10] P. Ledesma and J. Usaola. Doubly fed induction generator model for transient stability analysis. IEEE Transaction on Energy Conversion, 20(2):388-397, June 2005. ISSN 08858969.

[11] A. S. Neto, S. L. A. Ferreira, J. P. Arruda, F. A. S. Neves, P. A. C. Rosas, and M. C. Cavalcanti. Reduced order model for grid connected wind turbines with doubly fed induction generators. In Industrial Electronics, 200\%. ISIE 2007. IEEE International Symposium on, pages 2655-2660, Vigo, June 2007.

[12] R. Pena, J. C. Clare, and G. M. Asher. Doubly fed induction generator using back-to-back PWM converters and its application to variable-speed wind-energy generation. In Electric Power Applications, IEE Proceedings -, volume 143, pages 231-241, May 1996. 